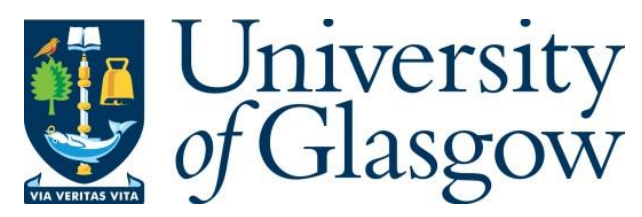

Shah, N. K. and Kontis, K. (2017) Flow-Control Effectiveness of Convergent Surface Indentations on an Aerofoil at Low Reynolds Numbers. In: 2017 AIAA Aviation Forum, Denver, CO, USA, 05-09 Jun 2017, ISBN 9781624105012.

There may be differences between this version and the published version. You are advised to consult the publisher's version if you wish to cite from it.

http://eprints.gla.ac.uk/145053/

Deposited on: 31 July 2017

Enlighten - Research publications by members of the University of Glasgow http://eprints.gla.ac.uk 


\title{
Flow-Control Effectiveness of Convergent Surface Indentations on an Aerofoil at Low Reynolds Numbers
}

\author{
Neel K. Shah ${ }^{1}$ \\ The University of Manchester, Manchester, M13 9PL, U.K. \\ and \\ Konstantinos Kontis ${ }^{2}$ \\ University of Glasgow, Glasgow, G12 8QQ, U.K.
}

\begin{abstract}
Passive flow control on aerofoils has largely been achieved through the use of protrusions such as vane-type vortex generators. Consequently, innovative flow-control concepts should be explored in an effort to improve current component performance. Therefore, experimental research has been performed at The University of Manchester to evaluate the flow-control effectiveness of a novel type of vortex generator made in the form of a surface indentation. The surface indentation has a trapezoidal planform. A spanwise array of indentations has been applied in a convergent orientation around the maximum-thickness location of the upper surface of a NACA-0015 aerofoil. The aerofoil has been tested in a twodimensional set-up in a low-speed wind tunnel at an angle of attack (AOA) of $3^{\circ}$ and a chordbased blockage-corrected Reynolds number $\left(\operatorname{Re}_{\text {corr }}\right)$ of $\sim 2.70 \times 10^{5}$. The baseline model has been found to suffer from a long laminar separation bubble (LSB) at low AoA. The application of the indentations at low AoA has considerably shortened the separation bubble. The indentations achieve this by shedding up-flow pairs of streamwise vortices. Despite the considerable reduction in bubble length, the increase in leading-edge suction due to the shorter bubble is limited by the removal of surface curvature and blockage (increase in surface pressure) caused locally by the convergent indentations. Furthermore, the up-flow region of the vortices, which locally weakens the pressure recovery around the trailing edge of the aerofoil by thickening the boundary layer, also contributes to this limitation. Due to the conflicting effects of the indentations, the changes in the pressure-lift and pressure-drag coefficients, i.e., $c_{1, p}$ and $c_{d, p}$, respectively, are small. Nevertheless, the indentations have improved $c_{1, p}$ and $c_{d, p}$ beyond the uncertainty range, i.e., by $\sim 1.3 \%$ and $\sim 0.3 \%$, respectively, at $3^{\circ}$ AoA. The wake measurements show that turbulence intensity and Reynolds stresses have considerably increased in the indented case, thus implying that the indentations increase the viscous drag on the model. In summary, the convergent indentations are able to reduce the size of the LSB, but conversely, they are not highly effective in enhancing $c_{1, p}$ and $\mathrm{c}_{\mathrm{d}, \mathrm{p}}$ at the tested Re.
\end{abstract}

\section{Nomenclature}

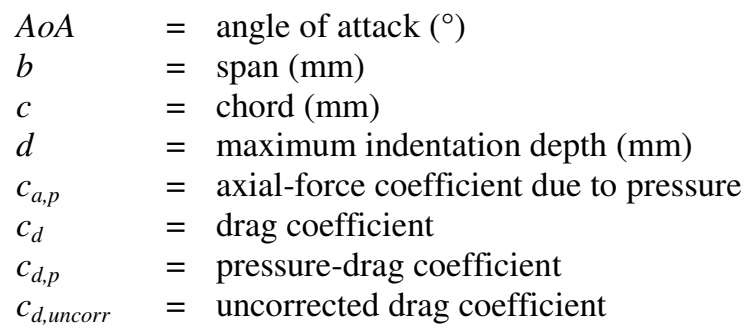

\footnotetext{
${ }^{1}$ Research Engineer, R\&D Department, Polypipe Civils Ltd., North Road, Loughborough, LE11 1LE.

${ }^{2}$ Professor of Aerospace Engineering, School of Engineering, University of Glasgow, Glasgow, G12 8QQ, AIAA Fellow.
} 


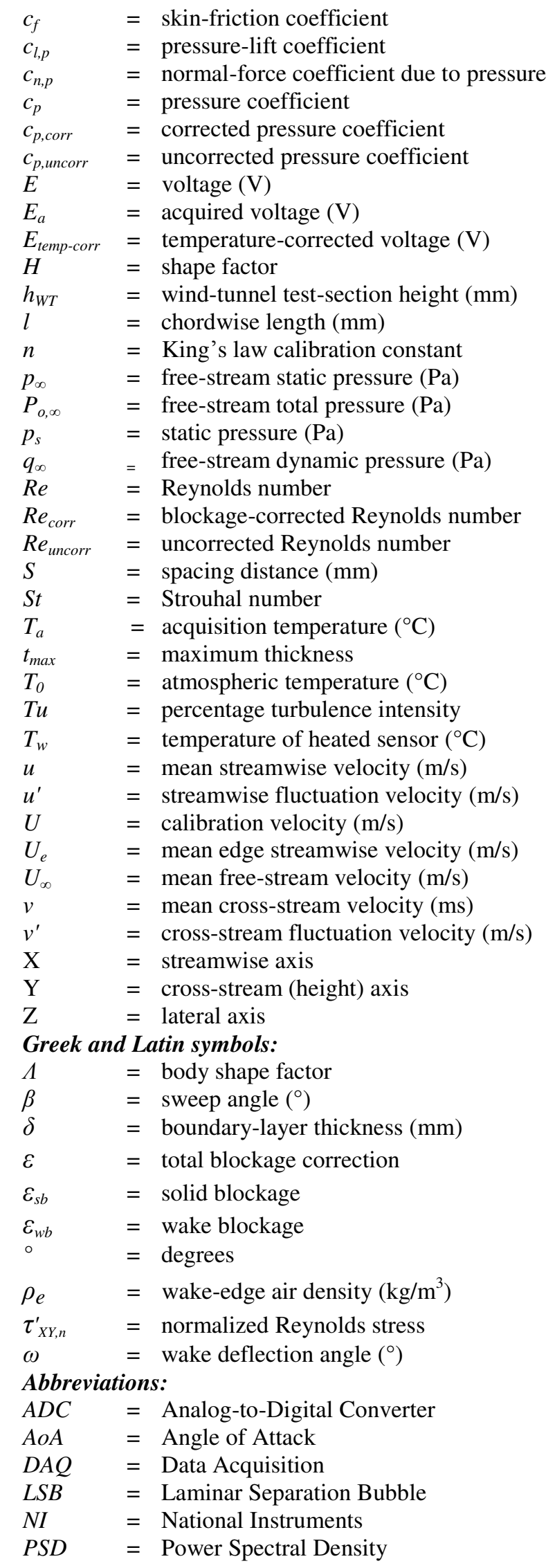

American Institute of Aeronautics and Astronautics 


$\begin{array}{ll}R M S & =\text { Root Mean Square } \\ T E & =\text { Trailing Edge } \\ V G & =\text { Vortex Generator } \\ V I & =\text { Virtual Instrument } \\ 2 D & =\text { Two Dimensional } \\ 3 D & =\text { Three Dimensional }\end{array}$

\section{Introduction}

$\mathrm{O}$ $\mathrm{NE}$ of the most important research endeavours in the aerospace industry involves aerodynamic flow control in order to increase the efficiency of aero-vehicle components. For future aircraft, the applications of innovative, effective, and efficient flow and noise control technologies will be highly critical in creating solutions for the increasing needs and demands of improved capacity, safety, mobility, efficiency, and environmental friendliness (reduced emissions and noise). ${ }^{1}$ Flow separation degrades system performance by causing significant deviations from inviscid pressure distributions. Hence, flow-separation control remains extremely important for many technological applications of fluid mechanics because of its ability to reduce the associated large energy losses and performance limitations. ${ }^{2}$ In addition, multi-disciplinary issues are increasingly playing an important role in modern aircraft design. Competitive pressures in the civil-aircraft industry are driving aircraft designers towards low-cost solutions. This means compromises have to be made in aerodynamic design; thus, considerations must be given for certain aircraft system configurations featuring flows that are either separated or close to separation, for example, a high-lift wing utilising a single flap. One practical solution lies in the use of flow-control devices to provide an expanding degree of freedom in the design optimization process. ${ }^{3,4}$

Boundary-layer separation is especially common at low Reynolds numbers (Re) at which laminar flow is prevalent. ${ }^{5}$ Several aerofoil applications operate in the low Re regime, including micro air vehicles, high-altitude aircraft, compressor and low-pressure-turbine blades, and wind turbines. Typically, many of these aerofoils operate at a chord-based Re of less than $1.0 \times 10^{6}$ and experience a laminar separation bubble (LSB) for angles of attack (AoA) less than the stall angle. ${ }^{6}$ The LSB is formed slightly downstream of the beginning of the adverse-pressure gradient, where the laminar boundary layer separates and forms an unstable shear layer that rapidly transitions to a turbulent shear layer. Subsequent turbulent mixing and entrainment of high-speed fluid particles may, in some circumstances, cause the layer to reattach itself to the surface as a turbulent layer. Provided that the high-speed fluid entrained into the wall region supplies sufficient energy to maintain the circulating motion against dissipation, a small isolated separation zone (LSB) forms on the surface (see Fig. 1). Downstream of the point of reattachment, the newly formed turbulent boundary layer is capable of negotiating quite severe adverse-pressure gradients without separation. The precise conditions for the occurrence of separation, transition, and reattachment depend mainly on the Reynolds number, pressure distribution, surface curvature and roughness, and free-stream turbulence. ${ }^{4,7,8}$

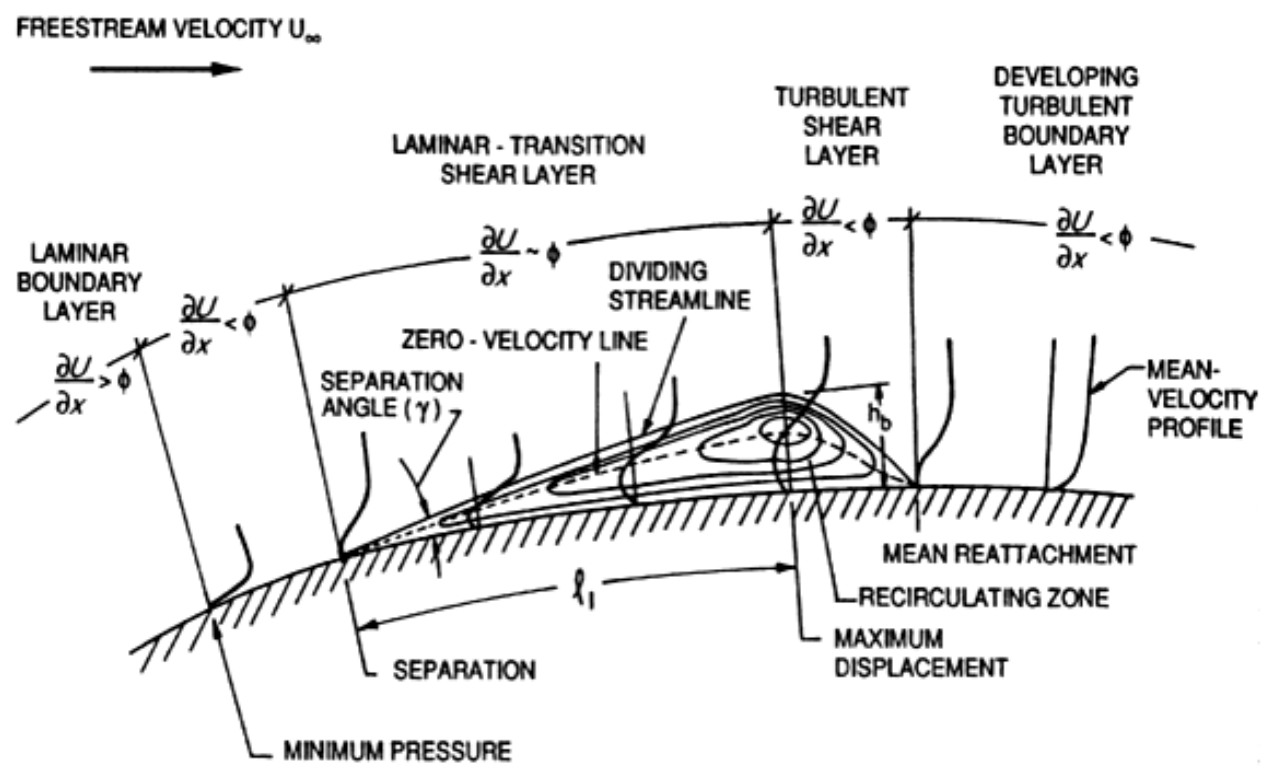

Figure 1. Flow around a laminar separation bubble (LSB). ${ }^{4}$

American Institute of Aeronautics and Astronautics 
The presence of a long separation bubble, which extends over several percent of the aerofoil chord, suppresses the leading-edge suction pressures on the aerofoil. Therefore, a long bubble significantly limits the aerofoil's lift and increases the pressure drag. ${ }^{4,9}$ Moreover, although short separation bubbles have been experimentally shown to have little effect upon the aerofoil's lift, they can create a thicker turbulent boundary layer that results in a significant drag increase. ${ }^{6}$ Therefore, the primary goal of flow control at low Re is to reduce or eliminate the separation bubble in order to increase lift and reduce drag.

Past and current aero-vehicles have benefited from passive flow-control techniques. These techniques commonly consist of vortex generators (VGs) that produce streamwise vortices to bring higher momentum to the near-wall flow, and thus enable it to overcome the adverse-pressure gradient. Nevertheless, although passive VGs have commonly been used to delay separation in turbulent boundary layers, they have rarely been used for the same purpose in laminar boundary layers. Furthermore, these VGs commonly consist of protrusions, such as vanes inclined at an angle to the oncoming flow. As a result, novel aerodynamic concepts to control flow separation should be researched in an endeavour to advance current aerodynamic-performance levels. Therefore, an experimental study has been conducted at The University of Manchester using a low-speed open-return wind tunnel to investigate the effectiveness of convergent surface indentations as a flow-control device.

\section{Experimental Model and Set-up}

\section{A. Test Model}

The NACA-0015 aerofoil has been chosen for this research due to its simple design. Moreover, at low Re, this aerofoil suffers from the detrimental effects of a LSB at low AoA. Hence, the effectiveness of the indentations in controlling this flow phenomenon can be evaluated.

The test model has a chord (c) of $250.0 \mathrm{~mm}$, span (b) of $450.0 \mathrm{~mm}$, and a maximum thickness ( $\left.\mathrm{t}_{\max }\right)$ of $37.5 \mathrm{~mm}$. The indentations have been located around the maximum-thickness location, i.e., 0.30c, of the aerofoil's upper surface. In order to size the depth of the indentations, initial boundary-layer measurements were performed in a natural-transition (baseline) condition at this location and a chord-based blockage-corrected $\operatorname{Re}\left(\operatorname{Re}_{\text {corr }}\right)$ of $\sim 2.70 \mathrm{x}$ $10^{5}$. The boundary-layer thickness at $0.30 \mathrm{c}$ of the baseline aerofoil at $3^{\circ} \mathrm{AoA}, \delta_{\mathrm{b}, 3 \mathrm{AoA}}$, was found to be $\sim 0.63 \mathrm{~mm}$.

\section{Application of the Indentations}

The surface indentations investigated in the current study entail a trapezoidal planform. The indentations have been chosen to be located around the $\mathrm{t}_{\max }$ location on the aerofoil to allow indentations of adequate depth to be machined. Furthermore, since this location is just downstream of the maximum suction region, it has been proposed that this location should facilitate the shedding of high-strength vortices by the indentations, which would increase their flow-control effectiveness. The shedding of counter-rotating streamwise vortices by such indentations was confirmed by this author in a previous study (Ref. 10).

The indentations have been designed to be a straight cut across a curvature. Hence, the depth of the indentation increases from its leading edge (at $\sim 0.21 \mathrm{c}$ ) to its maximum-depth location at $0.3 \mathrm{c}$, and then decreases towards its trailing edge (at $\sim 0.38 \mathrm{c}$ ). This variation in depth is illustrated by the sketch in Fig. 2.

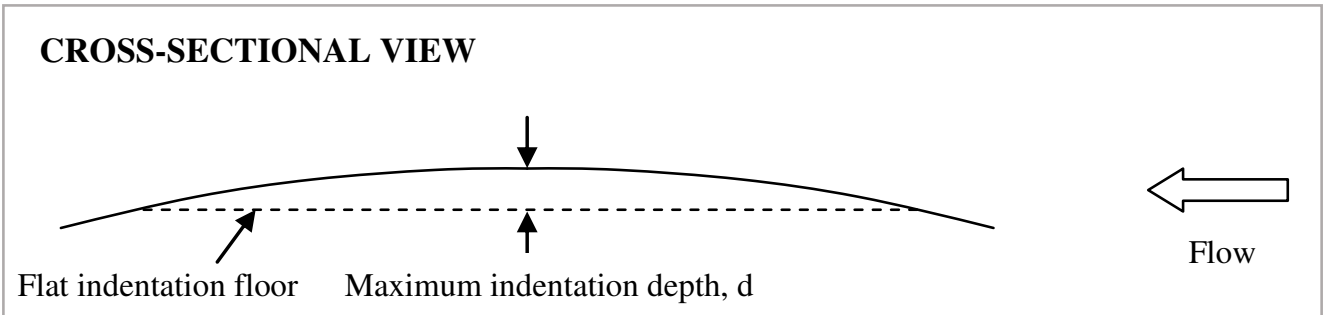

Figure 2. Cross-sectional view of the surface indentation

The discussion of indentation depth in this paper generally refers to the maximum indentation depth, $\mathrm{d}$. The various geometrical parameters of the indentations have been defined in Fig. 3. In order to test different sets of geometrical parameters, a two-dimensional (2D) spanwise slot has been milled on the upper surface of the model. The slot allows interchangeable inserts, each with a spanwise array of indentations of a particular set of geometrical parameters, to be mounted on the model for testing. The results presented in this paper correspond to a set of geometrical parameters consisting of a maximum-indentation-depth-to-local-baseline-boundary-layer-thickness 
$\left(\mathrm{d} / \delta_{\mathrm{b}, 3 \mathrm{AoA}}\right)$ ratio of $\sim 0.8$, chordwise length of $42.1 \mathrm{~mm}$, spacing-to-chordwise-indentation-length $(\mathrm{S} / \mathrm{l})$ ratio of 0.5 , and sweep angle $(\beta)$ of $15^{\circ}$. Also, the 2 swept edges of the indentation have been spaced apart by $10 \mathrm{~d}$ at the apex (trailing edge) of the indentation in an attempt to mitigate mutual interference between the counter-rotating vortices.

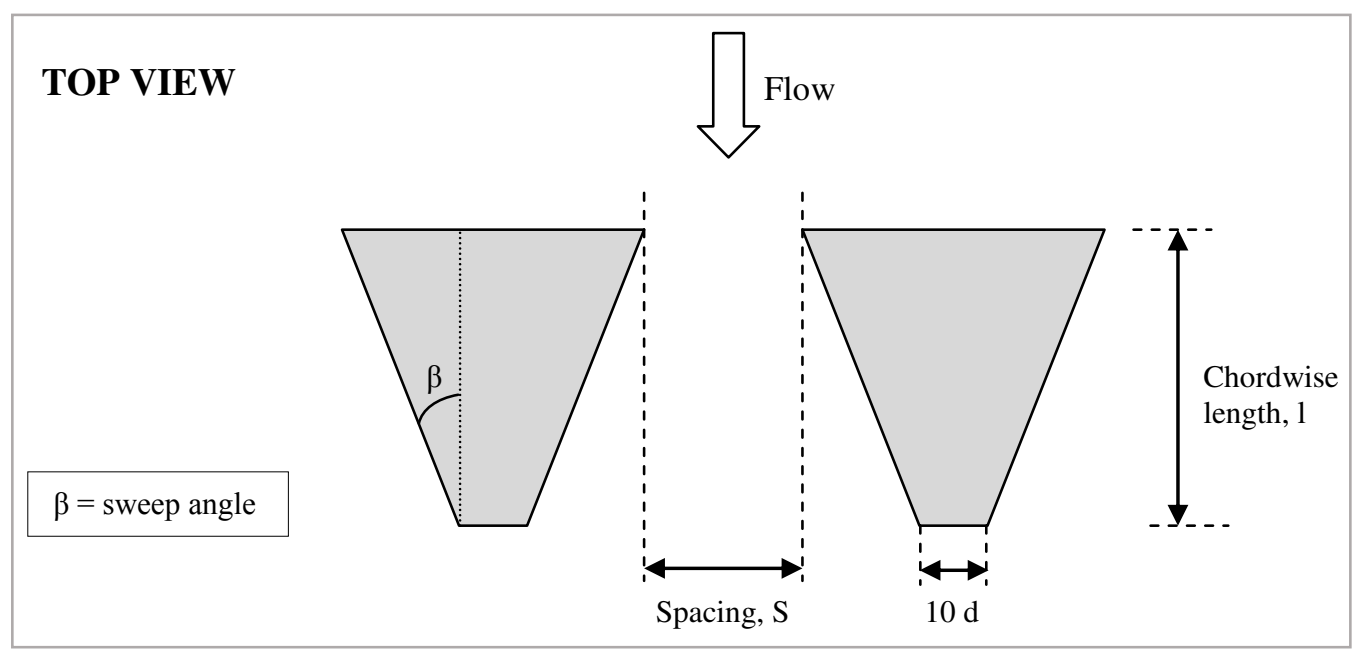

Figure 3. Definition of surface-indentation parameters

The indentations have been tested in a convergent orientation to the oncoming flow. This is demonstrated in Figs. 3 and 4. The upper surface of the insert has been profiled accordingly to maintain the original NACA-0015 curvature. A spanwise array of threaded holes with a spacing of $5 \mathrm{~mm}$ has been made on the base of the slot to allow the insert to be placed at different spanwise positions on the model. However, as shown in Fig. 4, this also prerequisites the insert to be made wider than the model span.

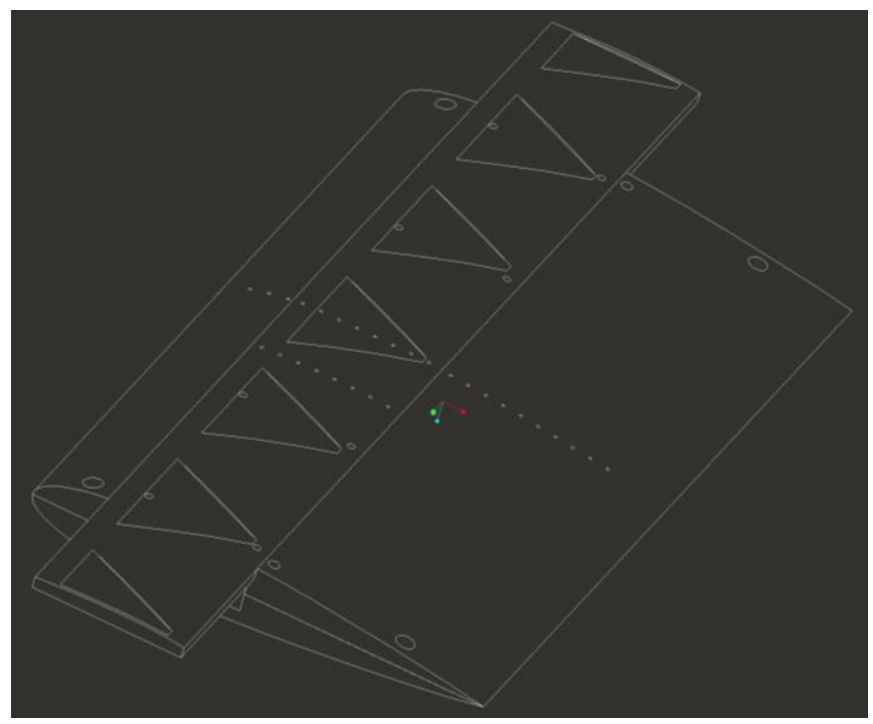

Figure 4. Application of convergent indentations

\section{Pressure Tappings}

Tappings for the measurement of surface pressure have also been opened to the atmosphere along the centreline (mid span) of the model. The upper surface of the insert also has pressure tappings to allow a complete pressure distribution to be acquired as well as enable pressure measurements to be made in the vicinity of the indentations. The tappings are located at $0.002 \mathrm{c}, 0.030 \mathrm{c}, 0.060 \mathrm{c}$, and then every $0.040 \mathrm{c}$ up to $0.900 \mathrm{c}$. Thus, there are a total of 24 tappings on the upper surface of the model. In order to evaluate the effect of the three-dimensional (3D) flow on 
the surface pressure, the insert has pressure tappings at two additional spanwise stations, which are shown in Fig. 5. The insert has 8 tappings at each of the three spanwise stations. There are also 24 pressure tappings along the centreline of the aerofoil's lower surface with the same chordwise spacing and locations as those on the upper surface. Thus, pressure distributions consisting of 48 points can be acquired. The tappings have a diameter of 1.6 $\mathrm{mm}$. In order to allow a trivial connection to the tappings, hypodermic tubes with a wall thickness of $0.25 \mathrm{~mm}$ have been inserted into the pressure tappings, thus giving an effective tapping diameter of $1.1 \mathrm{~mm}$.

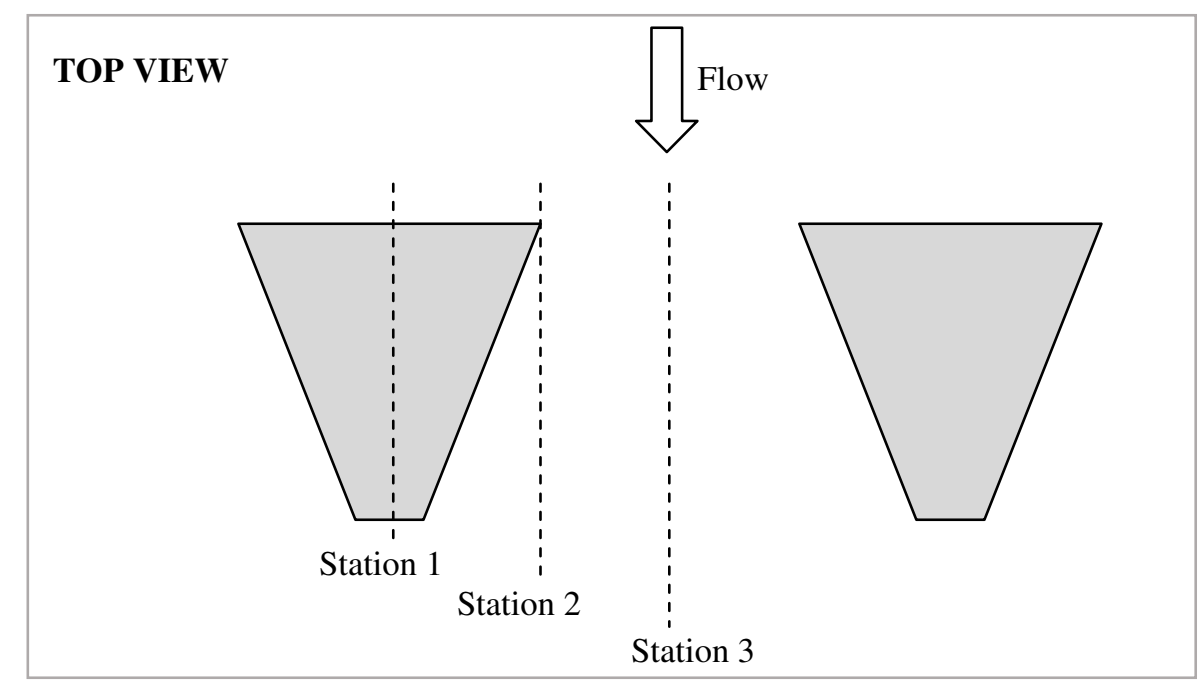

Figure 5. Designation and location of spanwise stations for various measurements

\section{B. Low-speed Wind Tunnel}

The wind tunnel used for the experiments generates a maximum velocity of $25 \mathrm{~m} / \mathrm{s}$ in the test section. The dimensions of the test section are a length (X) of $1420 \mathrm{~mm}$ by a height $(\mathrm{Y})$ of $455 \mathrm{~mm}$ by a width (Z) of $455 \mathrm{~mm}$. Therefore, since the model span is $450 \mathrm{~mm}$, the current study can be considered to be 2D. Optical access is provided through clear-Perspex windows in the ceiling and side walls of the test section. The test section also benefits from an in-house customised system of two traverses that also allows a supported probe to be rotated in the streamwise cross-stream (X-Y) plane (as defined in Fig. 6). This is necessary for boundary-layer measurements on curved surfaces such as aerofoils. The traverse uses a protractor with a resolution of $1^{\circ}$ for setting the angle of the probe support. As shown by the schematic in Fig. 6, the test section's top window has a $915 \mathrm{~mm}$ (X) by $20 \mathrm{~mm}(\mathrm{Z})$ slot to allow various probes to be traversed along the model's mid-span (centreline). The free-stream turbulence near the start of the test section is $\sim 0.3 \%$.

\section{Model Set-up}

The model has been supported horizontally in the test section by its side windows via two $12 \mathrm{~mm}$-diameter rods, i.e., one on each side of the model. The rods are centred at $0.250 \mathrm{c}$, thus, the rotation point of the model should be located around its aerodynamic centre. The set-up of the model in the test section can be seen from the schematic in Fig. 6.

\section{Measurement Techniques and Set-up}

\section{A. Test Conditions}

All experiments have been performed at $3^{\circ} \mathrm{AoA}$ and a $\mathrm{Re}_{\text {corr }}$ of $\sim 2.70 \times 10^{5}$ based on the free-stream velocity, $\mathrm{U}_{\infty}$, and aerofoil chord, c. This Re has been chosen to allow close comparison with the results of Ref. 5, where a passive spanwise groove on a NACA-0015 aerofoil has been used at a Re of $\sim 2.38 \times 10^{5}$. 


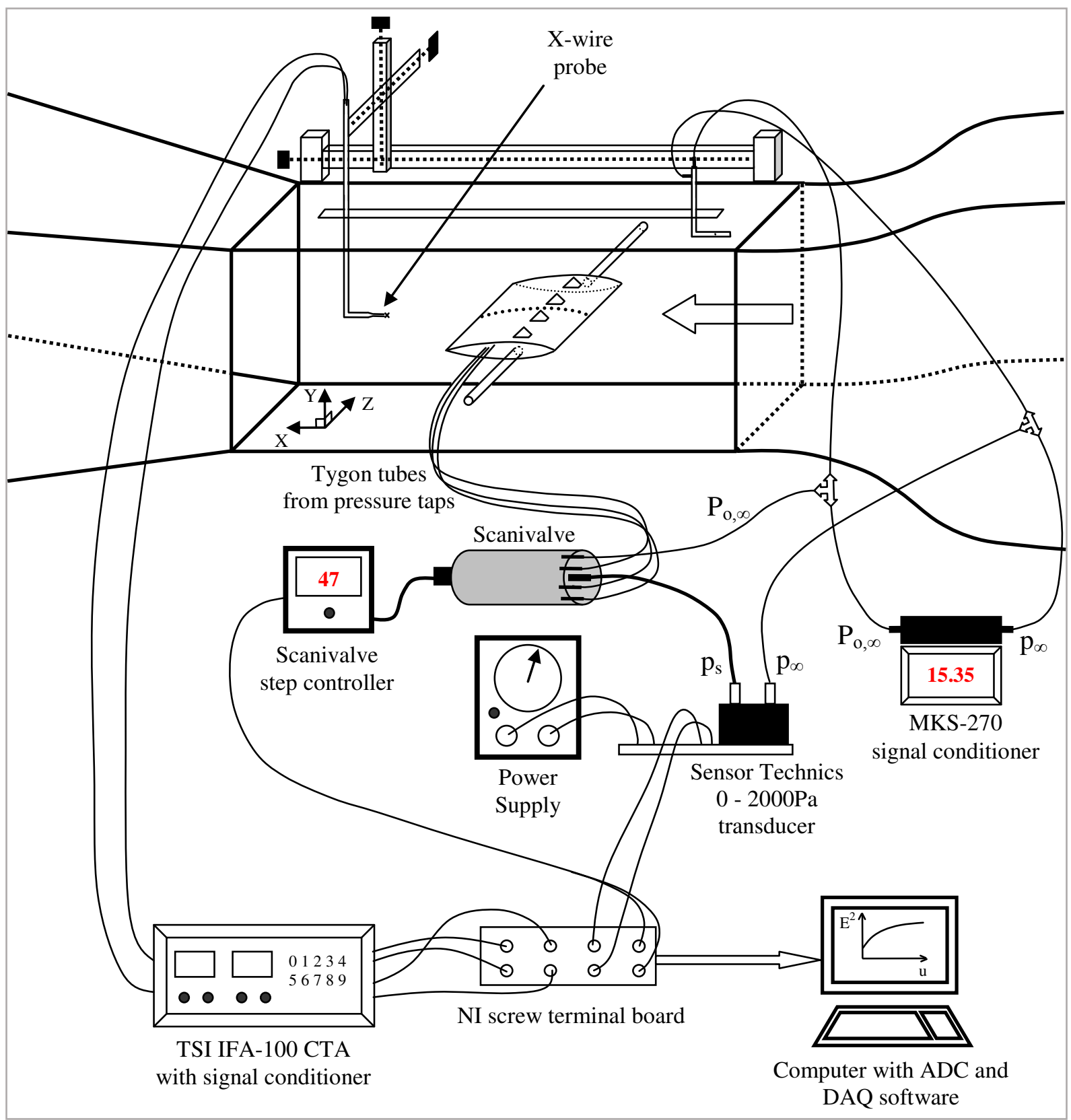

Figure 6. Schematic representing the set-up used for the surface-pressure and cross-hotwire measurements

\section{B. Blockage Corrections}

In the current work, solid blockage, $\varepsilon_{\mathrm{sb}}$, and wake blockage, $\varepsilon_{\mathrm{wb}}$, have been evaluated and accounted for using the following standard corrections for 2D incompressible flow. These corrections are given in Ref. 11, unless specified otherwise.

The solid blockage $\left(\varepsilon_{\mathrm{sb}}\right)$ can be estimated as:

$$
\varepsilon_{s b}=\Lambda \sigma
$$

where $\Lambda$ is the body shape factor, and equal to 0.305 for the NACA-0015 aerofoil, ${ }^{11}$ and, 


$$
\sigma=\frac{\pi^{2}}{48}\left(\frac{c}{h_{W T}}\right)^{2}
$$

where, $\mathrm{c}$ is the model chord, and, $\mathrm{h}_{\mathrm{wT}}$ is the test-section height.

The wake blockage $\left(\varepsilon_{\mathrm{wb}}\right)$ can be estimated as:

$$
\varepsilon_{w b}=\frac{c / h_{W T}}{4} c_{d, \text { uncorr }}
$$

where $\mathrm{c}_{\mathrm{d} \text {,uncorr }}$ is the uncorrected drag coefficient.

Hence, the estimation of the total blockage correction, $\varepsilon$, is:

$$
\varepsilon=\varepsilon_{s b}+\varepsilon_{w b}
$$

This allows the Re and pressure coefficient, $c_{p}$, to be corrected through Eqs. (5) and (6):

$$
\begin{gathered}
\operatorname{Re}_{\text {corr }}=\operatorname{Re}_{\text {uncorr }}(1+\varepsilon) \\
c_{p, \text { corr }}=c_{p, \text { uncorr }}(1-2 \varepsilon)+2 \varepsilon(\text { Ref. 12) }
\end{gathered}
$$

where the subscripts corr and uncorr denote corrected and uncorrected, respectively.

\section{Measurement Techniques}

A variety of measurement techniques have been employed to provide both qualitative and quantitative results. Oil-flow visualisation has also been performed on the suction surface to characterise the salient surface-flow features, for example, changes in the extent of flow separation. In order to understand the changes made by the indentations to the flow properties, detailed surveys of surface static pressure using pressure tappings, and nearwake characteristics using cross-hotwire anemometry have been made along the mid-span (centreline) of the test model's suction surface.

Due to the generation of counter-rotating vortices, a 3D flow-field would develop downstream of the indentations. Hence, the insert containing the spanwise array of indentations has been traversed laterally to locate the middle indentation at different spanwise positions relative to the model's centreline to enable the surveys to be made at various spanwise stations of the indentation. The aerodynamic performance (for this study, pressure lift and pressure drag) of the model has been evaluated from surface-pressure measurements.

\section{Oil-flow Visualization}

The clarity of the surface-flow patterns and representation of the true flow has been found to be dependent on the recipe of the oil mixture. The composition of the standard mixture comprised of paraffin, titanium (IV) oxide $\left(\mathrm{TiO}_{2}\right.$ ) powder, and colour glow in a respective $10 \mathrm{ml}: 1.5$ grams : 1.5 grams ratio. This composition has been altered by varying the amount of $\mathrm{TiO}_{2}$ powder and colour glow for the same amount of paraffin to obtain the best results. The most suitable mixture has been determined by experimental verification.

A relatively simple set-up has been used for the oil-flow visualization experiments. It has consisted of a light source located on either side of the test section to evenly and adequately illuminate the model surface, and a conventional Canon digital camera, with a 1/2.5" CCD containing approximately 8 million pixels, located above and perpendicular to the model's streamwise - lateral (X-Z) plane.

\section{Surface-pressure Measurements}

Each pressure tapping has been connected to a port on a Scanivalve (made by Scanivalve Corporation) using flexible Tygon tubes with an internal diameter of $1.02 \mathrm{~mm}$. The Scanivalve used in this study consists of 48 ports for 
pressure inputs and a single output port. The Scanivalve has a stepper motor that connects its single output to each of the 48 inputs from the tappings pneumatically and sequentially. Since there are a total of 64 tappings on the model, two Scanivalves have been used. The experimental set-up is illustrated by the schematic in Fig. 6.

The pneumatic output of the Scanivalve has been fed to a single Sensor Technics HCX-series differential transducer with a $0-2000 \mathrm{~Pa}(\sim 0.7-4.5$ volts $)$ range, whose voltage output has been transmitted through a National Instruments' (NI) screw terminal board (SCB-68) to a NI 12-bit 16-channel analog-to-digital-converter (ADC) board (PCI-MIO-16E-1) for digitized acquisition of 20,000 samples at a rate of 5000Hz by a LabVIEW virtual instrument (VI). The data have been acquired sequentially since the Scanivalve needs to be stepped to connect one input port at a time to the single output port. The free-stream static pressure, $\mathrm{p}_{\infty}$, from the Pitot-static tube (located upstream of the model) has been connected to the second port of the differential transducer for use as the reference pressure. Thus, the voltage output of the transducer is in the corrected form, i.e., corresponding to:

$$
p_{s}-p_{\infty}
$$

where $\mathrm{p}_{\mathrm{s}}$ is the static pressure on the surface of the model.

$\mathrm{c}_{\mathrm{p}}$ has been calculated using Eq. 8:

$$
c_{p}=\frac{p_{s}-p_{\infty}}{q_{\infty}}
$$

where $\mathrm{q}_{\infty}$ is the free-stream dynamic pressure at the location of the Pitot-static tube.

The free-stream total pressure, $\mathrm{P}_{0, \infty}$, from the Pitot-static tube has been connected to the first port on the Scanivalve, thus the voltage output of this port corresponds to $\mathrm{q}_{\infty}$ at the Pitot-static tube location. Based on the calibration constants of the transducer, the LabVIEW VI calculates $\mathrm{q}_{\infty}$, and thus, the free-stream velocity, $\mathrm{U}_{\infty}$, at the location of the Pitot-static tube at the start of the sequence. Through the remainder of the sequence, the VI presents the pressure coefficient, $\mathrm{C}_{\mathrm{p}}$, at each tapping in real time. After scanning all the ports on the Scanivalve, the VI outputs a data file containing the 20,000 pressure samples at each tapping and the time-averaged $\mathrm{C}_{\mathrm{p}}$ distribution. The signals from the Pitot-static tube have been also channelled using T-junctions to a MKS Baratron manometer to monitor the consistency of the free-stream dynamic pressure, $\mathrm{q}_{\infty}$, throughout the entire pressure scan.

Distributions of pressure and skin-friction coefficients of the upper and lower surfaces of an aerofoil can be integrated to calculate the normal and axial force coefficients, i.e., $c_{n}$ and $c_{a}$, respectively. Since skin friction, $c_{f}$, has not been measured in the current work, only the normal and axial force coefficients due to surface pressure, i.e., $c_{n, p}$ and $c_{a, p}$, respectively, have been obtained. From $c_{n, p}$ and $c_{a, p}$, the pressure-lift and pressure-drag coefficients, i.e., $c_{1, p}$ and $\mathrm{c}_{\mathrm{d}, \mathrm{p}}$, respectively, have been calculated.

The total uncertainty in mean $\mathrm{c}_{\mathrm{l}, \mathrm{p}}$ and $\mathrm{c}_{\mathrm{d}, \mathrm{p}}$, based on a $95 \%$ confidence level, is $\sim+/-0.0065(\sim+/-2.2 \%)$ and $\sim+/-$ $0.0005(\sim+/-2.8 \%)$, respectively.

\section{Wake Measurements - Cross-hotwire Anemometry}

Wake surveys have been performed using cross-hotwire anemometry. The measurements have been performed at $0.250 \mathrm{c}$ downstream of the aerofoil's trailing edge. This near-wake location was chosen to ensure that the flow characteristics generated by the indentations in terms of Reynolds stress and turbulence intensity could be adequately captured by the cross-hotwire (X-wire) probe before being damped by the flow viscosity. Furthermore, since the test section walls restrain curvature of the flow streamlines, any change in the deflection angle of the wake due to the application of the indentations would not be sufficiently evident after a certain streamwise distance. In addition to centreline measurements, the wake measurements have also been performed at the two additional spanwise stations shown in Fig. 5, thus allowing correlation with the surface-pressure measurements.

The wake has been surveyed using a DISA miniature X-wire probe (Type 55P61) consisting of 4 $\mu$ m-diameter tungsten sensing elements that are approximately $1.4 \mathrm{~mm}$ long. The prongs on which the tungsten sensing elements are mounted are separated by approximately $1 \mathrm{~mm}$. The probe allows measurement of both velocity and direction of the flow within an effective probe yaw-angle range of $+/-45^{\circ}$. Therefore, similar to the limitation of a single-wire probe, the X-wire probe cannot be used for measuring reversed flow. Nevertheless, its results can provide evidence 
of reversed flow by indicating regions of low and near-constant velocity magnitudes. In such an occurrence, moreappropriate measurement techniques should be used for verification and measurement of reversed flow.

In this work, the resultant velocity and direction of the wake flow have been measured in the streamwise - crossstream (X-Y) plane (as defined in Fig. 6), i.e., the deflection angle of the wake flow has been measured. Thus, the mean streamwise velocity, $\mathrm{u}$, and mean cross-stream velocity, $\mathrm{v}$, have been evaluated. The probe has been traversed with a clustering towards the trailing-edge height of the model, i.e., the distance between the measurement points was decreased to a minimum of $0.5 \mathrm{~mm}$ as the model's trailing-edge height was approached.

The X-wire probe has been operated by a TSI IFA-100 (Model 150) anemometer in a constant-temperature mode with an overheat ratio of 1.8. The IFA-100 is equipped with an internal signal conditioner (TSI Model 157), which has a third-order Sallen-Key type (-18 db/octave roll-off) low-pass filter. In order to maximise utilization of the full output range (+/-5 volts) of the signal conditioner, and thus improve the measurement resolution, the acquired signals have been DC-offset by 1 volt and amplified by a factor of 10 . Preliminary measurements at a high sampling rate of $10,000 \mathrm{~Hz}$ showed that the bandwidth of the flow frequencies did not exceed 1,000 Hz. Hence, the sampling frequency for the primary measurements was chosen as $5,000 \mathrm{~Hz}$ for both sensors. In order to satisfy the Nyquist criterion, the signals have been low-pass filtered at 2,000 Hz. The conditioned signals have then been transmitted through a NI screw terminal board (SCB-68) to a NI 12-bit 16-channel ADC board (PCI-MIO-16E-1) for digitized acquisition of 20,000 paired voltage samples (simultaneous acquisition from sensors 1 and 2) using a LabVIEW VI. The schematic in Fig. 6 also illustrates the set-up used for the X-wire experiments.

The X-wire probe has been calibrated using the full-velocity-versus-yaw-angle calibration method described in Ref. 13. In this method, the output of the $\mathrm{X}$-wire is measured at a range of known velocities and yaw angles of the probe. In the current work, the probe has been calibrated from $0 \mathrm{~m} / \mathrm{s}$ to $20 \mathrm{~m} / \mathrm{s}$ in increments of $2 \mathrm{~m} / \mathrm{s}$. For each velocity, calibration has been performed from $-30^{\circ}$ to $+30^{\circ}$ at intervals of $3^{\circ}$.

The acquired voltages have been corrected for effects of temperature drift during calibration through the following equation:

$$
E_{\text {temp-corr }}=\left(\frac{T_{w}-T_{o}}{T_{w}-T_{a}}\right)^{n} \cdot E_{a}
$$

where,

$\mathrm{E}_{\text {temp-corr }}$ is the temperature-corrected voltage,

$E_{a}$ is the acquired voltage (re-scaled back from its amplified and offset state),

$\mathrm{T}_{0}$ is the atmospheric temperature during the overheat-ratio setting,

$\mathrm{T}_{\mathrm{w}}$ is the temperature of heated sensor, and,

$\mathrm{T}_{\mathrm{a}}$ is the jet temperature during the acquisition of each voltage pair during calibration.

Following this correction, the instantaneous resultant velocity and instantaneous deflection angle for each of the 20,000 pairs of voltage samples have been computed. Subsequently, each instantaneous resultant velocity has been decomposed into instantaneous streamwise and cross-stream velocities before calculating the streamwise fluctuation velocity, u', and cross-stream fluctuation velocity, v', of each voltage-sample pair of the time series. Therefore, at a specific physical point in the flow, the Power Spectral Density (PSD) and Reynolds stress, $\tau_{X Y}^{\prime}$, could be obtained. $\tau_{X Y}^{\prime}$ has been evaluated using the following equation:

$$
\tau_{X Y}^{\prime}=-\rho \overline{u^{\prime} v^{\prime}}(\text { Ref. 14) }
$$

Furthermore, the standard deviation in $\mathrm{u}^{\prime}$ and $\mathrm{v}^{\prime}$ also allows the percentage turbulence intensity, Tu, to be quantified through the following equation:

$$
T u=\left[\sqrt{\frac{1}{2}\left(\overline{u^{\prime 2}}+\overline{v^{\prime 2}}\right)} / U_{e}\right] x 100
$$

where $\mathrm{U}_{\mathrm{e}}$ is the mean streamwise velocity at the upper edge of the wake. 
The total uncertainties, based on a 95\% confidence level, of various wake quantities are presented in Table 1:

\begin{tabular}{|c|c|c|}
\hline \multicolumn{3}{|c|}{ Total uncertainty, +/- } \\
\hline \multirow{2}{*}{$\mathrm{u} / \mathrm{U}_{\mathrm{e}}$} & Upper wake edge & 0.0010 \\
\cline { 2 - 3 } & Wake core & 0.0025 \\
\cline { 2 - 3 } & Lower wake edge & 0.0150 \\
\hline \multicolumn{2}{|c|}{ Normalized probe position } & 0.001 \\
\hline \multicolumn{2}{|c|}{ Wake-deflection angle } & $0.39^{\circ}$ \\
\hline \multicolumn{2}{|c|}{ Normalized Reynolds stress (wake core) } & 0.0002 \\
\hline \multicolumn{2}{|c|}{ Percentage turbulence intensity (wake core) } & $0.2 \%$ \\
\hline $\begin{array}{c}\text { Normalized velocity } \\
\text { fluctuations }\end{array}$ & u-component & 0.0025 \\
\cline { 2 - 3 } & v-component & 0.0017 \\
\hline
\end{tabular}

Table 1. Total uncertainties of various quantities in the wake

The uncertainty in $\mathrm{u} / \mathrm{U}_{\mathrm{e}}$ at the remaining positions in the wake has been estimated by linearly interpolating between the wake-core and wake-edge uncertainties.

\section{Boundary-layer Measurements - Single-hotwire Anemometry}

For boundary-layer measurements, a DISA miniature single-hotwire probe (Type 55P11) with a $5 \mu \mathrm{m}$-diameter and $1.25 \mathrm{~mm}$-long tungsten sensing element has been used. The single-hotwire experiments have utilised the same TSI IFA-100 anemometer, data-acquisition set-up, signal-conditioning parameters, temperature-correction scheme, and sampling rate as those used for the cross-hotwire calibration and experiments. The only difference is that the single hotwire probe has been calibrated in accordance with King's law at a single yaw angle of $0^{\circ}$. King's law is defined as:

$$
E^{2}=n_{1}+n_{2} U^{n_{3}}
$$

where,

$\mathrm{E}$ is the voltage,

$\mathrm{n}_{1}, \mathrm{n}_{2}$, and $\mathrm{n}_{3}$ are the King's law calibration constants, and,

$\mathrm{U}$ is the calibration velocity.

Thus, the calibration constants $\mathrm{n}_{1}, \mathrm{n}_{2}$, and $\mathrm{n}_{3}$ have been found and used to obtain the velocity equating to the voltage output from the anemometer during the experiments. Figure 7 shows a typical calibration curve.

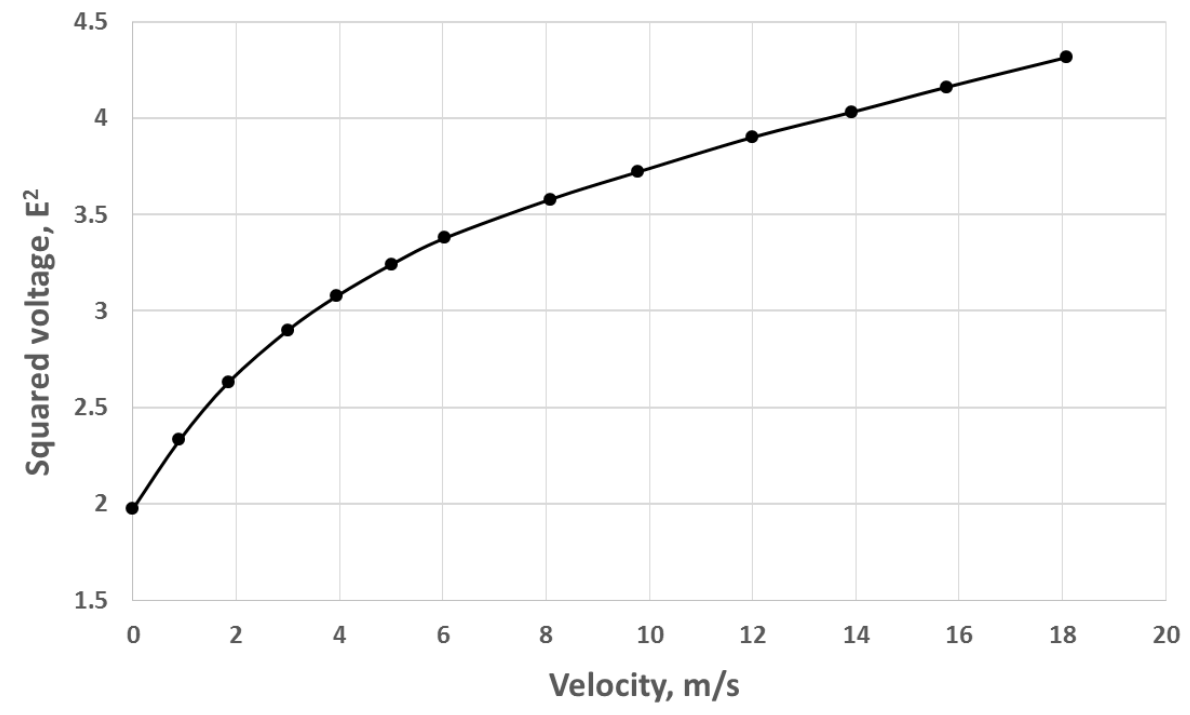

Figure 7. Typical single-hotwire calibration curve

11

American Institute of Aeronautics and Astronautics 
Based on a 95\% confidence level, the total uncertainty of the normalized probe position and non-dimensional root-mean-square (RMS) voltage is $+/-0.001$ and $+/-3.32$, respectively.

\section{Final Comments}

For the data presented in the next section, no discussion is made for cases where the uncertainty range of an indented-case result is overlapping with the uncertainty range of the baseline result by more than 50.0\%. For overlaps of less than $50.0 \%$, the result is still inconclusive, but the result is discussed only in the context of an indication of a possible change.

\section{Results and Discussion}

In this section, the results from different measurement techniques have been analysed and discussed to explain the effects of the convergent indentations on the aerofoil's aerodynamic performance and various flow quantities at selected spanwise stations at $3^{\circ}$ AoA. The measured quantities include the aerodynamic force coefficients, i.e., $\mathrm{c}_{1, \mathrm{p}}$ and $\mathrm{c}_{\mathrm{d}, \mathrm{p}}$, flow separation and reattachment locations (laminar-separation-bubble extents), surface static pressure, flow fluctuations over the surface of the aerofoil, and finally, the velocity, turbulence intensity, and Reynolds stress in the near wake. The baseline flow at $3^{\circ}$ AoA has been found to suffer from a long LSB. Therefore, the section focuses on the influence of the indentations on this flow phenomenon and downstream flow properties to elucidate their manipulation of the aerofoil's aerodynamic performance.

\section{A. Aerodynamic Force Coefficients}

In relation to the baseline case, the mean $\mathrm{c}_{1, \mathrm{p}}$ and $\mathrm{c}_{\mathrm{d}, \mathrm{p}}$ at $3^{\circ}$ AoA have improved by $\sim 0.017(\sim 5.7 \%)$ and $\sim 0.0009$ $(\sim 5.5 \%)$, respectively, at Station 2 , and by $\sim 0.020(\sim 6.8 \%)$ and $\sim 0.0011(\sim 6.3 \%)$, respectively, at Station 3 . However, the mean $\mathrm{c}_{1, \mathrm{p}}$ and $\mathrm{c}_{\mathrm{d}, \mathrm{p}}$ have degraded by $\sim 0.004(\sim 1.2 \%)$ and $\sim 0.0002(\sim 1.2 \%)$, respectively, at Station 1. The total uncertainty in mean $\mathrm{c}_{1, \mathrm{p}}$ and $\mathrm{c}_{\mathrm{d}, \mathrm{p}}$ is $\sim+/-0.0065(\sim+/-2.2 \%)$ and $\sim+/-0.0005(\sim+/-2.8 \%)$, respectively. Hence, there is a high level of uncertainty in $\mathrm{c}_{\mathrm{l}, \mathrm{p}}$ and $\mathrm{c}_{\mathrm{d}, \mathrm{p}}$ at Station 1 since their uncertainty ranges are overlapping with the corresponding baseline uncertainty range by $\sim 72.5 \%$ and $\sim 78.3 \%$, respectively. Thus, no comment can be provided about these changes. Hence, the only improvements in $\mathrm{c}_{\mathrm{l}, \mathrm{p}}$ outside the uncertainty range are the increments at Stations 2 and 3, which respectively reduce to a minimum increase (worst case) of $\sim 0.004(\sim 1.3 \%)$ and $\sim 0.007$ $(\sim 2.4 \%)$. The reduction in $\mathrm{c}_{\mathrm{d}, \mathrm{p}}$ at Station 3 is also outside the uncertainty range, thus providing a minimum reduction of $\sim 0.0001(\sim 0.8 \%)$ at this station. Furthermore, the overlap between the Station-2 and baseline $\mathrm{c}_{\mathrm{d}, \mathrm{p}}$ uncertainty ranges is only $\sim 2.1 \%$, thus providing a strong indication that $\mathrm{c}_{\mathrm{d}, \mathrm{p}}$ has also been slightly reduced at this station.

By only considering the changes that are outside the uncertainty range, the indentations provide a minimum spanwise-averaged improvement in $\mathrm{c}_{\mathrm{l}, \mathrm{p}}$ and $\mathrm{c}_{\mathrm{d}, \mathrm{p}}$ of $\sim 1.3 \%$ and $\sim 0.3 \%$, respectively. In summary, the application of the convergent indentations at $3^{\circ}$ AoA results in a small improvement in mean $c_{1, p}$ and $c_{d, p}$.

\section{B. Oil-flow Patterns}

As illustrated in Fig. 8, the oil-flow patterns on the baseline model show oil accumulation, i.e., a separation bubble, starting from $\sim 0.180 \mathrm{c}$. Along the centreline pressure taps, the separated flow reattaches at $\sim 0.560 \mathrm{c}$, thus the bubble extends over a significant streamwise length of $\sim 0.380 \mathrm{c}$, and is therefore a long separation bubble. The oilflow patterns in Fig. 9(a) clearly illustrate that the flow-field created by the indentations is highly 3D and complex. Nonetheless, the surface patterns are fairly uniform (across the model span) and discrete with the presence of fragmented bubble areas. These features are possibly generated by discrete flow structures such as streamwise vortices. This author showed in Ref. 10 that convergent indentations generate up-flow pairs of counter-rotating streamwise vortices from their swept edges. In the present study, footprints caused by vortex "scouring" are evident in the oil mixture immediately after the flow-reattachment location downstream of the indentations' apexes. The footprints, and thus the vortices, extend all the way to the aerofoil's trailing edge.

Although the indentations are submerged within the LSB, the indentations still affect the flow significantly. In particular, the indentation array has disrupted and fragmented a significant portion of the LSB. This suggests that the bubble height is less than the maximum depth of the indentations.

Despite the flow-field around and downstream of the bubble being 3D, the laminar separation line is reasonably 2D. Nonetheless, the disturbances generated by the indentations are also propagated upstream, thus the separation line has been shifted upstream by $\sim 0.030 \mathrm{c}$ in comparison to the baseline case, that is, from $\sim 0.180 \mathrm{c}$ to $\sim 0.150 \mathrm{c}$. As illustrared in Fig. 9(b), turbulent reattachment around Station 1 occurs approximately at the apex location, i.e., $0.410 \mathrm{c}$, which is $\sim 0.150 \mathrm{c}$ earlier compared to the reattachment on the baseline model. Since the bubble extends from $\sim 0.180 \mathrm{c}$ to $\sim 0.560 \mathrm{c}$ on the baseline model, the indentations provide a considerable reduction of $\sim 0.120 \mathrm{c}(\sim 32.0 \%)$ in 
its streamwise extent at Station 1. In addition to fragmenting the bubble immediately downstream of their apexes, the indentations have also eliminated a portion of the bubble in the region adjacent to them, i.e., from $\sim 0.250 \mathrm{c}$ to $\sim 0.380$ c, thus leading to locally attached flow here. In other words, the bubble in between spanwise Stations 2 and 3 sustains only upstream and downstream of chordwise locations aligned approximately with the adjacent leading edge and trailing edge of the indentations, respectively. Therefore, the single long separation bubble that exists in the baseline case has been broken into two separate shorter bubbles in the spanwise region adjacent to the indentations, i.e., in between Stations 2 and 3, thus effectively producing bi-camber at these stations. Since the first and second bubbles extend from $\sim 0.150 \mathrm{c}$ to $\sim 0.250 \mathrm{c}$ and $\sim 0.380 \mathrm{c}$ to $\sim 0.540 \mathrm{c}$, respectively, the total length of the prevailing bubble is similar as at Station 1, i.e., $\sim 0.260 \mathrm{c}$. However, the indentations have also distributed the bubble over a marginally larger streamwise extent around these two stations, i.e., by $\sim 0.010 \mathrm{c}$, in comparison to the baseline case. In summary, the bubble has been shortened by an equal amount across the model span. Therefore, the spanwise-averaged reduction in bubble length at $3^{\circ} \mathrm{AoA}$ is $\sim 0.120 \mathrm{c}(\sim 32.0 \%)$.

The observations at Stations 2 and 3 highlight a peculiar phenomenon; however, an attempt to explain its occurrence is made as follows. Figure 9 demonstrates that oil accumulates within the indentation. The accumulation here can be afforded because the vortex from the convergent indentations forms off the swept edge of the indentation and onto the normal surface adjacent to the indentations. Hence, the vortex fragments the bubble in between the indentations, and forces the first portion of the bubble to reattach. Furthermore, it can be clearly seen from the oil patterns that as the flow adjacent to the indentations approaches and goes past the indentations' trailing edge, it is drawn in towards the indentation. As mentioned above, the convergent indentations shed up-flow pairs of vortices, which converge together. This is because the swept edges of each indentation converges the flow towards the apex, hence the converging flow induces the adjacent vortices to draw closer to each other, that is, laterally away from Station 3 and towards Station 1. Consequently, the vortices cannot provide adequate mixing to fully transition the initial reattached flow between Stations 2 and 3, but rather reattach and transition the flow immediately downstream of the indentation's apex along Station 1. Thus, a second separation zone forms on the region adjacent to the apexes of the indentations.

It is important to note that the application of the oil mixture would reduce the effective depth of the indentations, thereby causing them to generate weaker disturbances / structures. Consequently, the flow-field features portrayed by the oil patterns, such as the reattachment points, vortex footprints, etc., might be slightly different (in terms of location, size, etc.) to those on a "dry" model.

\section{Surface Pressures}

A long LSB leads to a thicker turbulent boundary layer after reattachment, thus effectively decambering the aerofoil. Consequently, a long LSB weakens the pressure recovery towards the trailing edge and suppress the suction pressures near the leading edge. These pressure changes manifest as reduced lift and increased pressure drag.

The upper-surface $C_{p}$ distributions for the indented and baseline cases are given in Fig. 10. It can be noticed that the pressure distributions at Stations 2 and 3 (Fig. 10(b)) are similar to each other, and this is because of the presence of similar flow features along them (as depicted by the oil-flow patterns in Fig. 9). It is also evident that the suction near the leading edge of the aerofoil, i.e., from $\sim 0.002 \mathrm{c}$ to $\sim 0.170 \mathrm{c}$, has been augmented at Stations 2 and 3 . The average increase in this chordwise extent is $\sim 0.037(\sim 5.6 \%)$ higher than the baseline average. The indentations provide this benefit by reducing the extent of the long LSB. The oil-flow patterns in Fig. 9 show that reattachment aft of the first bubble at Stations 2 and 3 occurs at $\sim 0.250 \mathrm{c}$, and due to this reattachment process, the $\mathrm{C}_{\mathrm{p}}$ becomes higher than the baseline values from $\sim 0.180 \mathrm{c}$ at these two stations. Thus, the increased pressure recovery associated with the reattachment process begins before the reattachment location due to the down-washing streamlines around the rear portion of the bubble. Furthermore, significant pressure changes occur along the indentation, i.e., at Station 1, (see Fig. 10(a)) which would also influence the pressure at the adjacent stations. At Station 1, the pressure rises beyond the baseline values in the front portion of the indentation due to its converging geometry, i.e., the flow within the convergent indentation is forced to converge towards its apex. Consequently, this pressure rise is also transmitted to Stations 2 and 3. The $\mathrm{C}_{\mathrm{p}}$ is higher than the baseline values up to $\sim 0.350 \mathrm{c}$ and $\sim 0.330 \mathrm{c}$ at Stations 2 and 3 , respectively. The average $\mathrm{C}_{\mathrm{p}}$ in this increased-pressure region is higher than the baseline average by $\sim 0.024$ $(\sim 3.4 \%)$ and $\sim 0.019(\sim 2.6 \%)$ at Stations 2 and 3, respectively. However, due to the presence of the second bubble from $\sim 0.380 \mathrm{c}$ (as portrayed by the oil-flow patterns), the rate of pressure recovery in the increased-pressure region rapidly decreases from $\sim 0.290 \mathrm{c}$ and $\sim 0.270 \mathrm{c}$ at Stations 2 and 3, respectively. This is because the thickness of the second bubble changes the effective curvature locally. Furthermore, the rate of pressure recovery at Stations 2 and 3 is also reduced by the low pressure created around Station 1 as the flow approaches the apex of the indentations. Consequently, the attached-flow $\mathrm{C}_{\mathrm{p}}$ almost plateaus between $\sim 0.290 \mathrm{c}$ and $\sim 0.370 \mathrm{c}$, and in the process, it falls below the baseline values from $\sim 0.350 \mathrm{c}$ and $\sim 0.330 \mathrm{c}$ at Stations 2 and 3, respectively. Despite beginning to increase within 
the bubble, the $\mathrm{C}_{\mathrm{p}}$ remains lower than the baseline values up to $\sim 0.460 \mathrm{c}$ and $\sim 0.470 \mathrm{c}$ at Stations 2 and 3 , respectively. The average $\mathrm{C}_{\mathrm{p}}$ in this reduced-pressure region is $\sim 0.011(\sim 1.9 \%)$ and $\sim 0.018(\sim 3.2 \%)$ lower than the baseline average at Stations 2 and 3 , respectively. Downstream of $\sim 0.470 \mathrm{c}, \mathrm{C}_{\mathrm{p}}$ again recovers to higher values up to $\sim 0.580 \mathrm{c}$ since the flow undergoes a second reattachment, as detailed below.

The oil-flow patterns show that turbulent reattachment downstream of the second separation bubble occurs at $\sim 0.540 \mathrm{c}$. The start of the second reattachment process is marked by the increased rate of pressure recovery from $\sim 0.450 \mathrm{c}$; conversely, the end of the process is signified by a reduction in this rate aft of the reattachment location. Since the second reattachment occurs $\sim 0.020 \mathrm{c}$ upstream of the baseline reattachment location of $\sim 0.560 \mathrm{c}$, the indented-case $C_{p}$ rises slightly above the baseline values in between $\sim 0.500 \mathrm{c}$ and $\sim 0.580 \mathrm{c}$. In this chordwise region, the largest increase in $\mathrm{C}_{\mathrm{p}}$ occurs at the reattachment location of $\sim 0.540 \mathrm{c}$. Alternatively, the baseline $\mathrm{C}_{\mathrm{p}}$ becomes marginally greater downstream of its reattachment location of $\sim 0.560 \mathrm{c}$, that is, between $\sim 0.600 \mathrm{c}$ and $\sim 0.740 \mathrm{c}$. However, downstream of $\sim 0.740 \mathrm{c}$, the indented and baseline $\mathrm{C}_{\mathrm{p}}$ values are similar, and pressure recovers at approximately a constant gradient towards the trailing edge. Therefore, although the indentations fragment the bubble at Stations 2 and 3 by a significant and similar amount as at Station 1, i.e., $\sim 0.120$ c $(\sim 32.0 \%)$, they do not improve the pressure recovery at the trailing edge of the aerofoil at these two stations.

It is worthwhile to note that although correlations between the oil-flow patterns and surface pressure have been attempted above, there might be a slight difference in the location and streamwise extent of the bubble during the oil-flow and surface-pressure experiments due to different levels of intrusiveness to the flow.

A few notable differences exist between the pressure distributions at Station 1 and Station 3. Firstly, the increase in the magnitude of the suction pressures near the leading edge is significantly less at Station 1 . Between $\sim 0.002 \mathrm{c}$ and $\sim 0.170 \mathrm{c}$, the magnitude of the average suction $\mathrm{C}_{\mathrm{p}}$ at Station 1 is only $\sim 0.010(\sim 1.5 \%)$ higher than the baseline average, while at Stations 2 and 3, the increment is $\sim 0.037(\sim 5.6 \%)$. Thus, only the latter increment is outside the average uncertainty range of $\sim+/-0.013(\sim+/-1.8 \%)$ over this chordwise extent. This suggests that the lack of curvature due to the presence of the indentation and the pressure increase around the front portion of the indentation caused by its converging geometry limit the recovery of the leading-edge suction along Station 1.

Secondly, the oil-flow patterns show that reattachment at Station 1 occurs approximately at the indentation apex, i.e., $\sim 0.410 \mathrm{c}$. Although this is downstream of the first reattachment at Station 3 , the region of increased pressure (compared to the baseline case) downstream of $\sim 0.180 \mathrm{c}$ extends further at Station 1, i.e., up to $\sim 0.370 \mathrm{c}$. The average $\mathrm{C}_{\mathrm{p}}$ over this chordwise region is $\sim 0.021(\sim 3.0 \%)$ higher than the baseline average. As seen from the oil-flow patterns in Fig. 9, the bubble around Station 1 has been contained within the area of the indentation. Hence, the converging geometry of the indentations (projection of the swept walls to the oncoming flow) increases the pressure within the bubble as well as on the area adjacent to the indentations, i.e., at Stations 2 and 3.

Thirdly, at Station 1, as the flow approaches the apex of the indentation, $\mathrm{C}_{\mathrm{p}}$ decreases rapidly starting from approximately the mid-length location of the indentation, i.e., $\sim 0.330 \mathrm{c}$, and reaches a minimum at the apex and reattachment location, i.e., $\sim 0.410 \mathrm{c}$, where it is $\sim 0.056(\sim 10.1 \%)$ lower than the baseline value. This suggests that the flow accelerates out of the indentation due to its converging swept walls. Therefore, $\mathrm{C}_{\mathrm{p}}$ at $\mathrm{Station} 1$ is lowered below the baseline values between $\sim 0.370 \mathrm{c}$ to $\sim 0.460 \mathrm{c}$ by an average amount of $\sim 0.012(\sim 2.1 \%)$. As observed before, the oil-flow patterns show that turbulent reattachment at Station 1 occurs approximately at the apex of the indentation. Turbulent reattachment normally provides high pressure due to the down-washing streamlines around the rear portion of the separation bubble. Hence, an important note to be made here is that reattachment at Station 1 has occurred in a region of low pressure. Therefore, the increase in pressure over the front portion of the indentation and then a significant decrease over the rear portion suggests that the pressure field around the indentation is dominated by its converging geometry rather than the presence of the prevailing bubble over it.

Finally, since the flow at Station 1 reattaches earlier compared to the baseline case as well as the second reattachment at Stations 2 and $3, \mathrm{C}_{\mathrm{p}}$ at Station 1 recovers to higher values earlier, that is, from $\sim 0.470 \mathrm{c}$. Conversely, the baseline $\mathrm{C}_{\mathrm{p}}$ becomes higher than the Station-1 values aft of $\sim 0.580 \mathrm{c}$, that is, immediately downstream of its reattachment location of $\sim 0.560 \mathrm{c}$, and remains slightly higher up to the trailing edge. Thus, the pressure recovery at Station 1 is weaker than all other cases. This is because the up-flow region between a pair of counter-rotating vortices, which thickens the boundary layer, should be located at this station. The weaker pressure recovery should also limit the recovery of suction at the leading edge at this station. In summary, in comparison to the baseline case, the pressure recovery at the trailing edge, i.e., downstream of $\sim 0.740 \mathrm{c}$, is unaltered at Stations 2 and 3 , while it has slightly weakened at Station 1.

The leading-edge suction pressures in the indented case could have also been augmented by the more-upstream located separation bubble. The thickness distribution of an aerofoil strongly influences its aerodynamic characteristics because it affects the pressure distribution and boundary-layer character. ${ }^{15}$ If the indentations do not affect the height of the shifted separation bubble, the effective body shape of the aerofoil would be altered so that the 
effective maximum thickness of the aerofoil would now be located forward of the baseline location. This has the effect of reducing the upper-surface pressure near the leading edge. ${ }^{16}$

Overall, there are four regions in which the surface pressure has been modified, that is, augmentation of the leading-edge suction due to the shorter and shifted separation bubble, the high-pressure region around and adjacent to the front portion of the indentation followed by a low-pressure region around and adjacent to the indentation apex, and a high-pressure region caused by the earlier turbulent reattachment.

On a side note, despite the presence of a long bubble, it can be noticed that the baseline pressure does not plateau within the bubble as expected. This is because the taps provide disturbances to the bubble. This means that the surface-pressure differences between the baseline and indented cases would be greater in the absence of the taps. Nonetheless, the baseline pressure distribution shows increased pressure recovery between $\sim 0.460 \mathrm{c}$ and $\sim 0.620 \mathrm{c}$, which depicts the reattachment process. Therefore, the taps have not fully tripped the flow along them.

\section{Boundary-layer Thickness}

References 17 and 18 show that the boundary layer is thickened in the up-flow region between a pair of streamwise vortices because the vortices sweep up low-momentum fluid from the near-surface in this spanwise region. The thicker boundary layer would lead to a weaker pressure recovery. As discussed earlier, the up-flow region in this investigation should be located at Station 1. Alternatively, in the down-flow region of streamwise vortices, the vortices sweep down higher-momentum fluid towards the surface from a higher height in the boundary layer. This leads to a thinner boundary layer, which would subsequently improve the pressure recovery in this spanwise region. For the current study, based on the width of the vortex footprints in the oil-flow patterns, the downflow region should be located close to Station 2. The pressure distribution in Fig. 10(a) shows that the pressure recovery at Station 1 is slightly weaker compared to that on the baseline aerofoil downstream of $\sim 0.580 \mathrm{c}$; however, the pressure recovery has not been affected at Stations 2 and 3.

Velocity profiles measured using single hot-wire anemometry reveal small changes to the boundary-layer thickness. Since a hotwire with a single sensor has been used in a 3D flow-field, its streamwise velocities are not accurate as the direction of the flow is unknown. However, the profiles provide some indication of the boundarylayer thickness since the velocities at the edge become constant. Figure 11 illustrates the development of the boundary-layer thickness along the upper surface at all three spanwise stations. It is evident that the boundary layer has been thickened at all three stations, with the greatest degradation at Station 1. More interestingly, the results show that the rate of boundary-layer growth has decreased considerably aft of $\sim 0.620 \mathrm{c}$ at Station 2 - near the trailing edge, the boundary-layer thickness at this station is similar to that on the baseline model. Since the streamwise vortices diffuse and grow in diameter as they spiral downstream, it suggests that they grow to an adequate diameter by $\sim 0.620 \mathrm{c}$ to begin thinning the boundary layer at Station 2 with their downward flow. However, the oil-flow patterns in Fig. 9 do not provide clear evidence of this, and additionally, the pressure recovery near the trailing edge at Station 2 is similar to that at Station 3, which has a thicker boundary layer aft of $\sim 0.620 \mathrm{c}$.

The velocity profiles at $\sim 0.900 \mathrm{c}$ are plotted in Fig. 12 , and show that the boundary layer has been thickened at Station 1 by $\sim 1.60 \mathrm{~mm}$ in relation to the baseline case, thus degrading the trailing-edge pressure recovery at this station. In addition to the vortex up-flow, the boundary layer could have also been thickened at Station 1 by the blockage caused by the converging geometry of the indentation, which manifests as high pressure around the front area of the indentation. The thickened boundary layer at the trailing edge decambers the aerofoil slightly in this spanwise region. This alters the pressure distribution around the aerofoil to yield less lift through a reduction in the magnitude of the suction pressures near the leading edge. The combination of weaker leading-edge suction with a weaker pressure recovery at the trailing edge also results in higher pressure drag. However, the indentations have also considerably shortened the separation bubble at Station 1, i.e., by $\sim 32.0 \%$. Additionally, the laminar flow also separates at an upstream location compared to the baseline case, i.e., at $\sim 0.150 \mathrm{c}$ instead of $\sim 0.180 \mathrm{c}$. Thus, the bubble has been shifted upstream by $\sim 0.030 \mathrm{c}$. The combined effect of this forward shift and a shorter bubble offsets the adverse effects of blockage, partial removal of surface curvature, and weaker trailing-edge pressure recovery, thus leading to marginally higher leading-edge suction at this station, as shown in Fig. 10(a). Overall, the net change in $\mathrm{c}_{\mathrm{l}, \mathrm{p}}$ and $\mathrm{c}_{\mathrm{d}, \mathrm{p}}$ at Station 1 is smaller than the amount that can be measured accurately in the current experiments.

Although the boundary layer at $\sim 0.900 \mathrm{c}$ has been thickened by $\sim 0.15 \mathrm{~mm}$ and $\sim 1.20 \mathrm{~mm}$ at Stations 2 and 3 , respectively, in comparison to the baseline thickness, the pressure recovery near the trailing edge has not degraded at these two stations. The reason for this is not yet fully known, however, one possibility is that the difference in surface pressure caused by a boundary layer that is only slightly thicker is not measurable by the transducer used. Furthermore, despite the degradation in boundary-layer thickness, the suction near the leading edge of the aerofoil has improved at Stations 2 and 3. This is because the streamwise vortices have broken the single bubble into two separate shorter bubbles at these two stations. Although the total length of the two shorter bubbles at Stations 2 and 
3 is equal to the length of the single bubble at Station 1, fragmenting the bubble into two separate shorter bubbles allows the leading-edge suction pressures at Stations 2 and 3 to recover to a greater extent, as evident in Fig. 10(b). This is because shorter bubbles have a smaller height, and therefore, their influence on the pressure distribution is less. ${ }^{6,9}$ Additionally, the two shorter bubbles at Stations 2 and 3 also create an effective body shape with a bicamber, which has been found by Bicamber Technology LLC to augment the upper-surface pressures. However, no publications have been found on this. Furthermore, Stations 2 and 3 are not affected by the lack of surface curvature as at Station 1, and the pressure in the chordwise region adjacent to the front portion of the indentation is not as high as that at Station 1. Thus, in contrast to Station 1, there is a small improvement in $\mathrm{c}_{\mathrm{l}, \mathrm{p}}$ and $\mathrm{c}_{\mathrm{d}, \mathrm{p}}$ at Stations 2 and 3.

\section{E. Flow Fluctuations}

As mentioned before, a single hot-wire has been used in a 3D flow-field. Hence, although the wire senses the resultant flow, the flow direction is unknown. The ambiguity in flow direction means that the correct calibration cannot be applied to the resultant voltage, and therefore, the magnitude of the resultant velocity cannot be obtained accurately. Consequently, the streamwise velocity component, and thus its RMS, cannot be ascertained accurately. However, the RMS of the acquired voltage signal (normalized with the RMS upstream of the model) provides evidence of the resultant fluctuations present at a particular chordwise location. It is important to note that the velocity RMS will be larger than the voltage RMS even after normalizing the two quantities due to non-linear effects through calibration. Therefore, the voltage RMS of the different cases only provides somewhat qualitative results.

The differences in voltage RMS between the indented and baseline cases at $\sim 0.140 \mathrm{c}, \sim 0.240 \mathrm{c}, \sim 0.320 \mathrm{c}$, and $\sim 0.410 \mathrm{c}$ are presented in Figs. 13 to 16, respectively. However, these differences are within the uncertainty range, thus a discussion of these results is not possible. However, as illustrated by Figs. 17 to 20, the difference between the Station-1 and baseline values increases markedly downstream of $\sim 0.410 \mathrm{c}$. Thus, the profiles indicate that the fluctuations at Station 1 begin to increase from approximately the apex of the indentation. Figure 17 shows that the fluctuations at this station peak at $\sim 0.500 \mathrm{c}$. At this chordwise location, the fluctuations increase towards the aerofoil surface, and at the first measurement point of $\mathrm{y} / \mathrm{c} \approx 0.0008$, the fluctuations are five times $(\sim 400.0 \%)$ higher than the baseline fluctuations. Therefore, although the oil-flow patterns in Fig. 9 show that reattachment occurs approximately at the indentation apex, i.e., $\sim 0.410 \mathrm{c}$, the turbulence is amplified downstream of the indentation apex. As shown by the pressure distribution in Fig. 10(a), the surface-pressure drops towards the apex and reaches a local minimum there. The fluctuations are damped at the reattachment location possibly due to this favourable pressure gradient. However, the reattached flow immediately meets an adverse-pressure gradient downstream of the apex, which possibly amplifies its fluctuations there. Another explanation is that the vortices shed by the indentation converge together slightly downstream of the indentation apex, with the resulting mutual interference between the two vortices leading to higher fluctuations there. The power spectral density (PSD) of the fluctuations at $\sim 0.410 \mathrm{c}$ along Station 1 demonstrates that the strength of the fluctuations increases away from the aerofoil surface before decreasing again (see Figs. 21 to 23). Hence, the most energetic fluctuations occur at a height of $\mathrm{y} / \mathrm{c} \approx 0.0016$, where the PSD level has been increased by a factor of $\sim 22.0$ in comparison to the baseline level at the same height from the surface. This indicates the presence of a small coherent structure rotating at $\sim 16 \mathrm{~Hz}$, i.e., a Strouhal number, St, of $\sim 0.25$, and located between $\mathrm{y} / \mathrm{c} \approx 0.0008$ and $\mathrm{y} / \mathrm{c} \approx 0.0025$ at $\sim 0.410 \mathrm{c}$. The oil-flow patterns in Fig. 9 show that the bubble is contained within the indentation, and that the flow immediately aft of the indentation's apex is attached. Thus, the flow structure located between $\mathrm{y} / \mathrm{c} \approx 0.0008$ and $\mathrm{y} / \mathrm{c} \approx 0.0025$ at the apex is likely to be the reversed rotational flow (transverse vortex) at the rear of the bubble over which the shear layer is downwashing before reattaching in the close vicinity of the apex.

The PSD of the fluctuations at $y / c \approx 0.0008$ at $\sim 0.500 \mathrm{c}$ (Fig. 24) shows that although the dominant fluctuations in the baseline case and at Station 1 occur at a similar frequency of $\sim 16 \mathrm{~Hz}$ (St of $\sim 0.25$ ), the dominant fluctuations at Station 1 are more energetic by a factor of $\sim 1.3$. Furthermore, the fluctuations have a higher energy content across the analysed spectra, especially up to $100 \mathrm{~Hz}$. The near-wall fluctuations at $\sim 0.500 \mathrm{c}$ at Stations 2 and 3 (Fig. 17) has also increased beyond the baseline values, for example, the RMS at the first measurement point at Station 3 is $\sim 152.0 \%$ higher. Hence, the greatest fluctuations occur at Station 1, possibly due to mutual interference between the pair of counter-rotating vortices. Since reattachment aft of the second bubble occurs at $\sim 0.540 \mathrm{c}$ at Stations 2 and 3, these higher fluctuations should be related to the local reattachment process. This argument is consolidated by the strength of the fluctuations downstream of the second reattachment at Station 3, i.e., at $\mathrm{y} / \mathrm{c} \approx 0.0008$ at $\sim 0.620 \mathrm{c}$, which decreases extensively as revealed by the PSD level in Fig. 25.

The PSD at the same height of $y / c \approx 0.0008$ at $\sim 0.500 \mathrm{c}$ and Station 2 (Fig. 26) illustrates that the dominant fluctuations there also occur at a St of $\sim 0.25$; furthermore, they also have higher energy content than the baseline level by a factor of $\sim 2.7$. In contrast to Station 1, the strength of the fluctuations at higher frequencies is 
insignificant, thus suggesting that the flow at $0.500 \mathrm{c}$ at Station 2 is affected mainly by a discrete flow structure. Since the oil-flow patterns show that the second LSB is present at $0.500 \mathrm{c}$ at this station, this frequency is likely to be the frequency of separation, for example, the frequency of the reversed rotational flow at the rear of the bubble. In relation to the previous observations, the most remarkable change in the PSD level at $\mathrm{y} / \mathrm{c} \approx 0.0008$ at $\sim 0.500 \mathrm{c}$ can be observed at Station 3. Figure 27 illustrates that the strength of the dominant fluctuations at this location have increased by a factor of $\sim 7.6$ compared to the baseline level. The dominant fluctuations still remain at a St of $\sim 0.25$, and since the middle of the second bubble (in terms of width) is located at Station 3, it gives further evidence that this frequency is the separation frequency. The PSD level is less at Station 2 because the edge of the bubble is located there; hence, interactions with the vortex / attached flow around Station 1 might affect the bubble fluctuations associated with reattachment at Station 2.

Therefore, the results illustrate that reattachment at Stations 2 and 3 is associated with lower fluctuations than at Station 1, but conversely, the dominant fluctuations at Stations 2 and 3 have higher energy content compared to Station 1. The lower fluctuations are possibly because transition to turbulent flow at Stations 2 and 3 occurs over two stages and / or the mutual interference between the shed vortices is not present at these stations. Furthermore, the lower energy content of the dominant fluctuations at the reattachment location at Station 1 (near the indentation apex) could have resulted from disruption to the reversed-rotational flow structure at the rear of the bubble by the converging walls of the indentation and / or up-flow of the shed vortices. As seen in Fig. 24, the mutual interference between the pair vortices along Station 1 leads to the creation of multiple flow structures at various frequencies and energy content downstream of the indentation apex, i.e., at $\sim 0.500 \mathrm{c}$.

As illustrated in Fig. 18, the near-wall baseline fluctuations at $\sim 0.620 \mathrm{c}$ are greater compared to those in the indented case since the baseline flow reattaches later compared to the indented case, i.e., at $\sim 0.560 \mathrm{c}$. Here, the indented case has similar RMS at all three spanwise stations. Figure 25 shows that the PSD level of $\sim 3.1$ for the dominant frequency in the baseline spectra at $\sim 0.620 \mathrm{c}$ has changed only slightly from the level at $\sim 0.500 \mathrm{c}$, i.e., $\sim 2.8$ (Fig. 24). Thus, the reattachment in the indented case is associated with more energetic fluctuations in comparison to the baseline case.

As shown by Figs. 19 and 20, the differences in the fluctuations are all again within the uncertainty range downstream of $\sim 0.620 \mathrm{c}$. Therefore, the largest increase in the flow fluctuations occurs around the respective reattachment locations of the baseline and indented cases (except at Station 1 where they peak slightly downstream). Since the fluctuations don't exhibit such behaviour at the end of the first bubble at $\sim 0.250 \mathrm{c}$ at Stations 2 and 3, it shows that the reattached flow aft of the first bubble has not fully transitioned to turbulent flow. The shape factor, $\mathrm{H}$, of the velocity profile at $\sim 0.320 \mathrm{c}$ (streamwise location in between the two bubbles) at Station 3 is $\sim 2.7$. (It must be noted again that this velocity profile has been measured by a single hot-wire sensor in a 3D flow-field, and thus the shape factor might not be exact). This value of $\mathrm{H}$ informs that the attached flow in between the two separation bubbles is still laminar. This is because the shed vortices, which enable the separated flow to initially reattach, are induced by the converging flow around Station 1 to move away from Stations 2 and 3, that is, towards Station 1. Subsequently, they are unable to provide the level of mixing required to fully transition the reattached laminar flow. Therefore, the flow cannot resist the adverse-pressure gradient, and thus separates again downstream to form a second separation bubble over which it undergoes full transition. Additionally, the formation of the second separation is possibly also aided by the lateral movement of the vortices, since they would entrain the flow in between Stations 2 and 3, and vector it towards Station 1. A comparable phenomenon was also found on a NACA0012 aerofoil with vane-type vortex generators in Ref. 19.

\section{F. Wake Flow}

\section{Near-wake Velocity}

The centreline near-wake profiles, i.e., at $\sim 0.250 \mathrm{c}$ downstream of the aerofoil trailing edge, show that the wake thickness at all three stations and the baseline case is similar, i.e., $y / c \approx 0.0970$ (see Fig. 28(a)). However, a closer look provided by Fig. 28(b) informs that the normalized streamwise velocities, u/ $\mathrm{U}_{\mathrm{e}}$, at Station 1 are slightly lower than the baseline values from $\mathrm{y} / \mathrm{c} \approx-0.0040$ to 0.0200 (where the uncertainty ranges of the two cases are not overlapping). Here, $\mathrm{y} / \mathrm{c}=0$ corresponds to the height of the trailing-edge. The normalized velocities are also lower at Station 2 , and additionally, the reduction here extends to a marginally greater height, i.e., from $\mathrm{y} / \mathrm{c} \approx-0.0040$ to 0.0300 . At Station 1, the slower flow is due to the vortex up-flow region where low momentum fluid is gathered. Two possibilities could have reduced $\mathrm{u} / \mathrm{U}_{\mathrm{e}}$ at Station 2 . Firstly, the vortices could have dissipated by the wake location, and the slower flow at Station 1 could have spread to Station 2 and reduced $u / \mathrm{U}_{\mathrm{e}}$ there. Alternatively, the vortices could have persisted into the wake and their component of lateral flow (in the spanwise direction) could have reduced $\mathrm{u} / \mathrm{U}_{\mathrm{e}}$ at Station 2. Compared to the baseline case, the maximum reduction in $\mathrm{u} / \mathrm{U}_{\mathrm{e}}$ at $S$ tations 1 and 2 is 
$\sim 0.0060(\sim 0.7 \%)$, and it occurs at the wake core location of $\mathrm{y} / \mathrm{c} \approx-0.0020$, where the uncertainty in $\mathrm{u} / \mathrm{U}_{\mathrm{e}}$ is $\sim+/$ $0.0025(\sim+/-0.3 \%)$. The average reduction in $\mathrm{u} / \mathrm{U}_{\mathrm{e}}$ at both stations from $\mathrm{y} / \mathrm{c} \approx-0.0040$ to 0.0200 is slightly lower than the maximum reduction, i.e., $\sim 0.0050(\sim 0.5 \%)$, while the average uncertainty range over this height region is $+/-0.0020(\sim+/-0.2 \%)$.

At Station 3, the normalized velocities are marginally higher (outside the uncertainty range) from $y / c \approx-0.0020$ to 0.0160 . The average increase in $\mathrm{u} / \mathrm{U}_{\mathrm{e}}$ over this height extent is $\sim 0.0060(\sim 0.8 \%)$ compared to the baseline average, and it is higher than the average uncertainty range of $\sim+/-0.0020(\sim+/-0.2 \%)$. It is not yet fully known what causes this slight velocity increment. Nonetheless, the boundary-layer-thickness results suggest that the vortices grow in diameter as they spiral downstream, and start to affect the boundary-layer thickness at Station 2 aft of $\sim 0.620 \mathrm{c}$. Thus, the vortices could have grown large enough by the wake-measurement location to affect the flow at Station 3 with their down-flow, which would sweep down higher momentum from a higher height in the wake to increase the streamwise velocity in low-momentum regions. The increase of $\sim 0.0040(\sim 0.5 \%)$ at the wake core $(\mathrm{y} / \mathrm{c} \approx-0.0020)$ is less than the average increase, and also within the uncertainty range of $\sim+/-0.0025(\sim+/-0.3 \%)$ at this location.

Overall, the slight differences in velocity across the three stations suggest that the near-wake has not fully mixed, that is, it is still 3D from the effects of the indentations. Nonetheless, despite the slight velocity changes, there is no measurable change in the wake core location of $\mathrm{y} / \mathrm{c} \approx-0.0020$.

\section{Wake-deflection Angle}

The deflection angles of the wake flow at the three spanwise stations and the baseline case are presented in Fig. 29. The uncertainty ranges are overlapping with each other; however, the overall trend at Station 1 indicates that the downward deflection there has been reduced in comparison to the baseline case. This is because of the vortex upflow region at this station. Conversely, there is an indication that the downward deflection around the trailing edge of the aerofoil at Station 3 has been increased, possibly because of the down-flow region of the vortices.

\section{Turbulence Intensity}

The turbulence intensity gives a measure of the turbulent fluctuations. In the current work, the percentage turbulence intensity, $\mathrm{Tu}$, has been calculated using Eq. 11. Figure 30 presents Tu in the near-wake. Compared to the baseline case, Tu is noticeably higher around the wake core at Station 1 due to the likely location and mutual interference of a pair of counter-rotating vortices around this station. Nonetheless, the similarity in Tu at Stations 1 and 2 implies that the flow has mixed at these two spanwise stations by the near-wake location. This is also evident by the similar non-dimensionalized streamwise velocities at these two stations around the wake core. The height extent over which the increased $\mathrm{Tu}$ is outside the uncertainty range $(\sim+/-0.2 \%)$ is from $\mathrm{y} / \mathrm{c} \approx-0.0200$ to 0.0060 . Over this region, the average Tu has increased from $\sim 7.8 \%$ in the baseline case to $\sim 8.3 \%$ at Stations 1 and 2 (i.e., by $\sim 6.6 \%$ ). The peak Tu, which is located at $\mathrm{y} / \mathrm{c} \approx-0.0150$, has increased from $\sim 8.2 \%$ in the baseline case to $\sim 8.7 \%$ at Stations 1 and 2 . Thus, the peak Tu at these two stations has increased by $\sim 5.8 \%$ in comparison to the baseline peak. The uncertainty ranges around the Station 3 and baseline peak values are overlapping by $\sim 33.0 \%$ (thus $\sim 0.1 \%$ overlap of percentage Tu); however, the overall trend at Station 3 indicates a small increase in Tu compared to the baseline case, with the peak value $\sim 4.1 \%$ higher. Since this is less than the Tu at Stations 1 and 2, it suggests that the near-wake flow has not fully mixed across the three spanwise stations.

As shown by Figs. 31 and 32, the increase in Tu is provided by higher RMS velocities in both streamwise and cross-stream directions, i.e., $\sqrt{\overline{\mathrm{u}^{\prime 2}}}$ and $\sqrt{\overline{\mathrm{v}^{\prime 2}}}$. The largest increase in the fluctuations occurs at Station 1 due to the likely mutual interference in between a pair of counter-rotating vortices at this station. The RMS velocities have been normalized with the wake-edge velocity. The v-component fluctuations in the wake are higher than the ucomponent fluctuations; in fact, the uncertainty ranges of the u-component fluctuations at all three stations are overlapping with the baseline uncertainty range. The uncertainty in $\sqrt{\overline{\mathrm{u}^{\prime 2}}} / \mathrm{U}_{\mathrm{e}}$ and $\sqrt{\overline{\mathrm{v}^{\prime 2}}} / \mathrm{U}_{\mathrm{e}}$ is $\sim+/-0.003$ and $\sim+/$ 0.002 , respectively. The height extent over which the increase in the v-component fluctuations is beyond the uncertainty range is greatest at Station 2, i.e., from $y / c \approx-0.0350$ to 0.0280 . At Station 1 , this extent reduces slightly to $\mathrm{y} / \mathrm{c} \approx-0.0350$ to 0.0210 . In relation to the baseline peak, the indented peak $\sqrt{\overline{\mathrm{u}^{\prime 2}}} / \mathrm{U}_{\mathrm{e}}$ and $\sqrt{\overline{\mathrm{v}^{\prime 2}}} / \mathrm{U}_{\mathrm{e}}$ fluctuations are higher by $\sim 0.003(\sim 3.6 \%)$ and $\sim 0.007(\sim 8.1 \%)$, and, $\sim 0.003(\sim 4.1 \%)$ and $\sim 0.006(\sim 6.3 \%)$ at Stations 1 and 2 , respectively. Therefore, the increase in the peak u- and v-component fluctuations at Stations 1 and 2 are similar. At Station 3, the u-component fluctuations are similar to the baseline values; however, the v-component fluctuations have somewhat increased outside the uncertainty range from $\mathrm{y} / \mathrm{c} \approx-0.0350$ to -0.0150 . The peak $\sqrt{\overline{\mathrm{v}^{\prime 2}}} / \mathrm{U}_{\mathrm{e}}$ value at 
this station is $~ 0.004(\sim 4.3 \%)$ higher than the baseline value. Hence, Station 3 experiences the smallest increase in the streamwise and cross-stream fluctuations, and therefore, the smallest increase in Tu.

Figures 33 to 35 plot the PSD at the height of maximum v-component fluctuations at Stations 1, 2, and 3, respectively. The dominant fluctuations at Stations 1 and 2 occur at a frequency of $\sim 485 \mathrm{~Hz}$ (St of $\sim 7.58$ ), while at Station 3, they occur at a slightly higher value of $\sim 515 \mathrm{~Hz}$ (St of $\sim 8.05$ ). These dominant fluctuations are most likely caused by the wing-tip vortices, which would be shed due to the finite gap of $\sim 2 \mathrm{~mm}$ between the model tips and side windows of the test section. It is clearly evident that the fluctuations at Station 3 have a higher energy content than at Station 2, i.e., PSD level of $\sim 159.0$ at Station 3 versus $\sim 97.5$ at Station 2 . The reason for this is not yet fully comprehended; however, it could have stemmed from the second flow reattachment on the aerofoil at Station 3, which is also associated with dominant fluctuations of higher energy content compared to Station 2 (compare Figs. 26 and 27). Nevertheless, Fig. 33 shows that the dominant v-component fluctuations in the wake at Station 1 have the highest energy content of the three stations, with a PSD level that is approximately twice the dominant level at Station 2, i.e., $\sim 186.0$.

It is important to note that the non-dimensional RMS velocities and Tu are similar at Stations 1 and 2 simply because of a higher wake-edge velocity at Station 1. Therefore, the dimensional fluctuations are larger at Station 1, which indicates that the effect of the pair of counter-rotating vortices is strongest there. In this context, in contrast to the observation based on Tu and the non-dimensional mean streamwise and RMS velocities, the spectral analysis shows that the wake flow at $\sim 0.250 \mathrm{c}$ downstream of the aerofoil trailing edge is still $3 \mathrm{D}$ across all three spanwise stations. As shown in the next section, this observation is confirmed by differences in Reynolds stresses across the three spanwise stations.

\section{Reynolds Stress}

The Reynolds stress gives a measure of the turbulent mixing in a flow. In the present work, the normalized primary Reynolds stress, $\tau_{X Y, n}^{\prime}$, has been calculated using Eq. 13:

$$
\tau_{X Y, n}^{\prime}=\frac{-\rho \overline{u^{\prime} v^{\prime}}}{\rho_{e} U_{e}^{2}}
$$

where $\rho_{\mathrm{e}}$ is the air density at the wake edge.

$\tau_{X Y, n}^{\prime}$ in the near-wake is plotted in Fig. 36. The profiles show the characteristic anti-symmetric distribution at first glance. However, even for the baseline profile, the magnitude of the peak positive Reynolds stress above the trailing-edge height is not equal to the magnitude of the peak negative Reynolds stress below the trailing-edge height due to the effects of curvature ${ }^{20}$ arising from a non-zero angle of attack. As expected, the figure demonstrates that the peak Reynolds stresses have been amplified at Stations 1 and 2, and thus informs that turbulent mixing has been enhanced at these two stations due to the counter-rotating vortices.

With respect to the baseline peak, the normalized peak positive Reynolds stresses at Stations 1 and 2 have been increased considerably, i.e., by $\sim 16.9 \%$ and $\sim 17.2 \%$, respectively. In comparison to the baseline case, the increase in the normalized Reynolds stress outside the uncertainty range of $\sim+/-0.0002$ extends from $\mathrm{y} / \mathrm{c} \approx 0.0160$ to 0.0200 and $\mathrm{y} / \mathrm{c} \approx 0.0170$ to 0.0280 at Stations 1 and 2 , respectively. The respective average increase is $\sim 15.3 \%$ and $\sim 20.8 \%$. Interestingly, unlike at Stations 2 and 3, the magnitude of the normalized peak negative Reynolds stress at Station 1 (at $\mathrm{y} / \mathrm{c} \approx-0.0160$ ) has also been amplified by $\sim 10.3 \%$; however, the uncertainty ranges of the Station-1 and baseline values are overlapping by $\sim 30.0 \%$. Thus, the mixing has possibly increased across the wake core at Station 1 . As reasoned for the turbulent fluctuations, the normalized positive Reynolds stress is slightly higher at Station 2 simply because of its lower wake-edge velocity. Hence, the dimensional Reynolds stress, $-\rho \overline{u^{\prime} v^{\prime}}$, is highest at Station 1. This is because the flow at this station would experience stronger mixing due to the likely location of the common up-flow region of a pair of counter-rotating vortices and the interference between them, while the flow at Station 2 would be affected by only one of the pair vortices.

The uncertainty ranges between the normalized Station-3 and baseline Reynolds stresses are overlapping throughout the profile, with an overlap of $\sim 45.0 \%$ occurring around the peak positive values. Therefore, the results only provide an indication that the peak positive Reynolds stress at Station 3 is larger than the baseline value. Nonetheless, this shows that the mixing in the wake of the indented aerofoil reduces with spanwise distance towards Station 3. 


\section{G. Summary}

Overall, the aerodynamic performance of the indented aerofoil at a low AoA of $3^{\circ}$ is affected by various effects, both favourable and adverse, on the surface flow and wake flow. At Station 1, on the one hand, the indentations shed counter-rotating vortices (despite being placed under the separation bubble) that increase mixing and hasten transition of the separated flow. This shortens the LSB by a considerable amount and shifts it upstream to provide aerodynamic benefits. On the other hand, the lack of curvature and high pressure (flow blockage) created locally by the indentations, and a thicker boundary layer and exacerbated pressure recovery around the trailing edge (due to the up-flow region of the pair vortices) all affect the aerodynamic performance adversely. Consequently, the improvement in leading-edge suction provided by the shorter bubble is compromised by the latter effects, and thus, the net result is that the aerodynamic force coefficients, $\mathrm{c}_{\mathrm{l}, \mathrm{p}}$ and $\mathrm{c}_{\mathrm{d}, \mathrm{p}}$, at Station 1 change by a smaller amount than their respective uncertainty ranges.

At Stations 2 and 3, due to the convergence of the vortices towards Station 1, the separation bubble has been fragmented into two separate shorter bubbles. The two separate bubbles, whose total length is equal to that of the single bubble at Station 1, should provide higher aerodynamic improvements since the height and adverse effect on the pressure distribution of shorter bubbles is less; furthermore, the two bubbles also create an effective bi-camber at Stations 2 and 3. Secondly, these two stations are located adjacent to the indentations, hence the aerofoil surface along them is unaltered; in addition, the high-pressure region adjacent to the indentations is shorter in streamwise extent compared to that at Station 1. Finally, the trailing-edge pressure recovery has not degraded at these two stations. Therefore, in comparison to Station 1, the leading-edge suction recovers to a greater level at Stations 2 and 3 , and this greater improvement increases $\mathrm{c}_{\mathrm{l}, \mathrm{p}}$ at these two stations by a larger amount than its uncertainty range. Moreover, due to the combination of stronger leading-edge suction and the high-pressure region at the rear of the model caused by the earlier reattachment, $\mathrm{c}_{\mathrm{d}, \mathrm{p}}$ at Station 3 also reduces by a larger amount than its uncertainty range.

By only considering the changes that are larger than the uncertainty range, the spanwise-averaged $\mathrm{c}_{\mathrm{l}, \mathrm{p}}$ and $\mathrm{c}_{\mathrm{d}, \mathrm{p}}$ of the indented case is better than the baseline $\mathrm{c}_{\mathrm{l}, \mathrm{p}}$ and $\mathrm{c}_{\mathrm{d}, \mathrm{p}}$ by at least $\sim 1.3 \%$ and $\sim 0.3 \%$, respectively, at this AoA. Nevertheless, turbulence intensity and Reynolds stress have also increased in the near wake of the indented model, which implies that the indented model suffers from higher viscous drag. Although the profile drag coefficient, $\mathrm{c}_{\mathrm{d} 0}$, can be calculated from the wake velocities, it has not been presented since the uncertainty ranges of all the indentedcase results are overlapping with the uncertainty range of the baseline result by more than $50.0 \%$. This is due to the significant uncertainty of $\sim+/-15.0 \%$ in $\mathrm{c}_{\mathrm{d} 0}$. Hence, it is not known if the aerodynamic efficiency of the indented model has improved or degraded at this AoA.

In summary, the convergent indentations are able to reduce the size of the LSB, but conversely, they are not highly effective in enhancing $\mathrm{c}_{\mathrm{l}, \mathrm{p}}$ and $\mathrm{c}_{\mathrm{d}, \mathrm{p}}$ at a $\mathrm{Re}_{\mathrm{corr}}$ of $\sim 2.70 \times 10^{5}$. However, the study in Ref. 21 , where the flowcontrol effectiveness of piezoelectric actuators was investigated at $0.250 \mathrm{c}$ and $0.500 \mathrm{c}$ (individually) on a NACA0015 aerofoil at a similar Re of $2.80 \times 10^{5}$, shows that $c_{1}$ was not improved at AoA below $6^{\circ}$. In another study, the effectiveness of a spanwise groove in controlling a LSB on a NACA-0015 aerofoil at $3^{\circ}$ AoA and a similar Re of $2.38 \times 10^{5}$ was examined in Ref. 5. Here, although the spanwise groove reduced the length of the separation bubble by $\sim 73.0 \%, c_{1}$ and $c_{d}$ were degraded by $\sim 11.0 \%$ and $\sim 27.7 \%$, respectively. In contrast, the convergent indentations used in the present study enhance $\mathrm{c}_{1, \mathrm{p}}$ by at least $\sim 1.3 \%$ on the same aerofoil at $3^{\circ}$ AoA. Nevertheless, in comparison to protruding VGs, the indentations do not perform as well. For example, in Ref. 6, wishbone VGs with a height of $\sim 0.3 \delta$ at $0.220 \mathrm{c}$ were utilized on a Liebeck LA2573A aerofoil. It was found that, at $4^{\circ}$ AoA and Re of $2.35 \times 10^{5}$, the wishbone VGs reduced $\mathrm{c}_{\mathrm{d}}$ by $\sim 38.0 \%$. Conversely, the convergent indentations manage to reduce $\mathrm{c}_{\mathrm{d}, \mathrm{p}}$ by only $\sim 0.3 \%$ at $3^{\circ}$ AoA. 


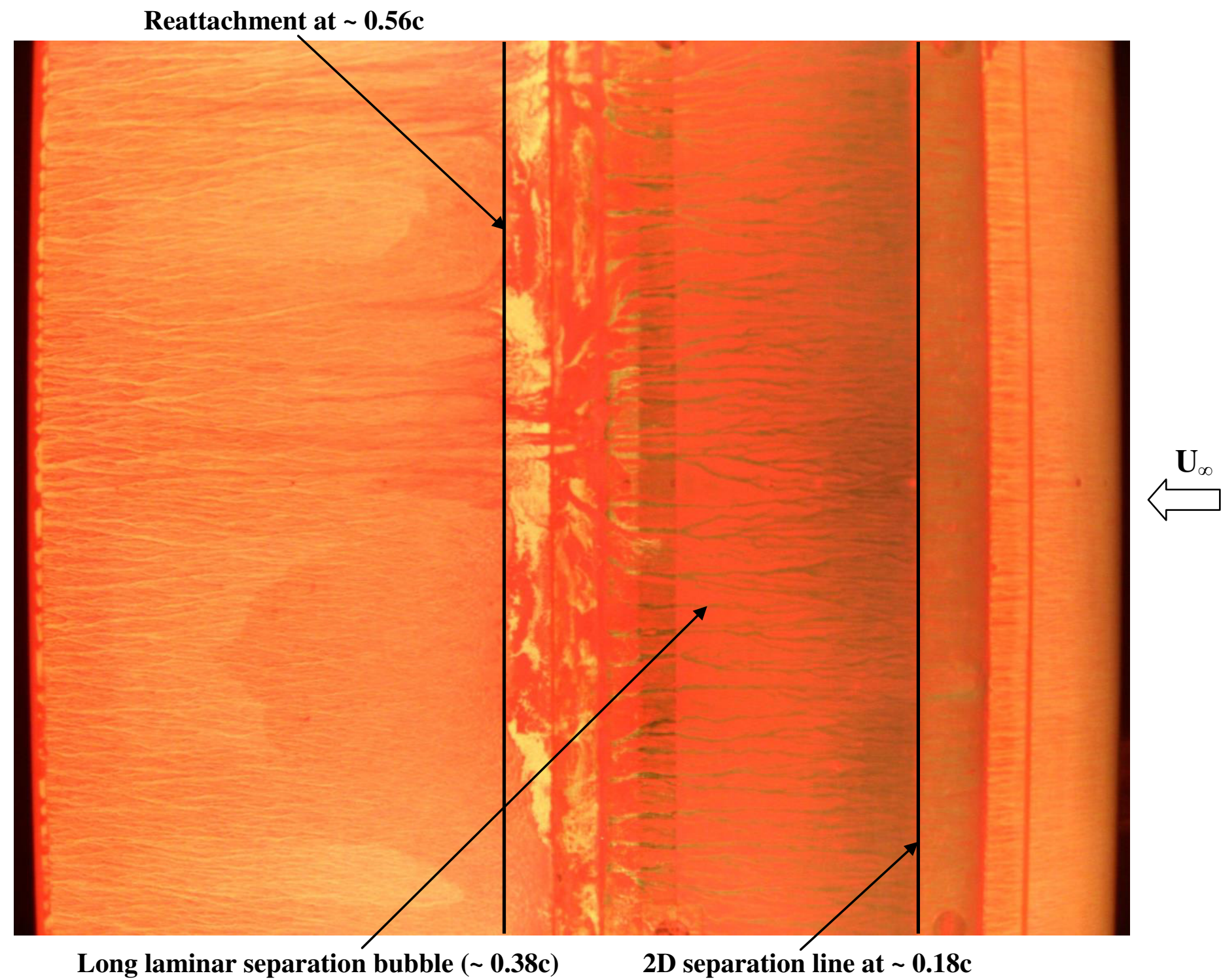

Figure 8. Oil-flow patterns on baseline model 


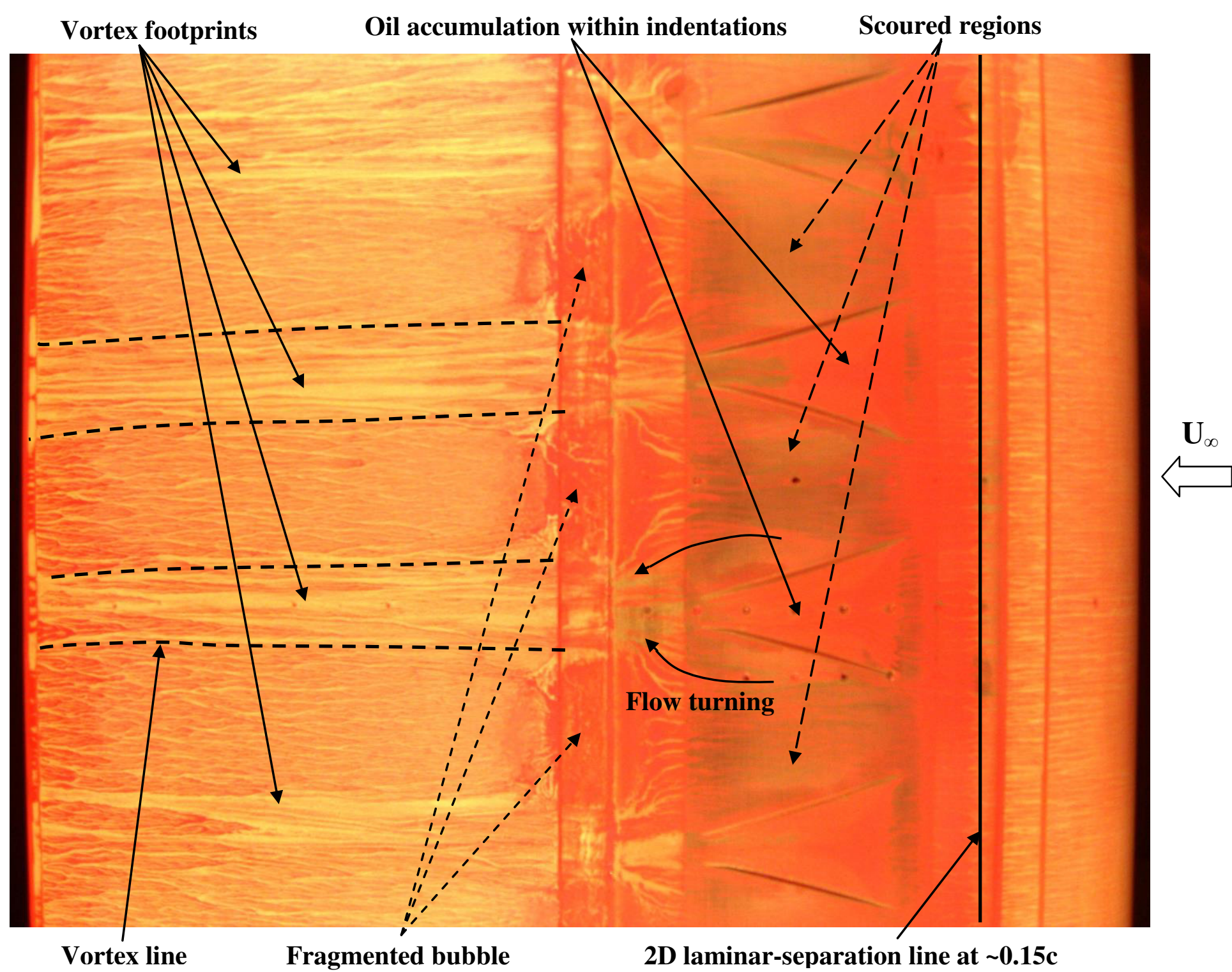

a) 


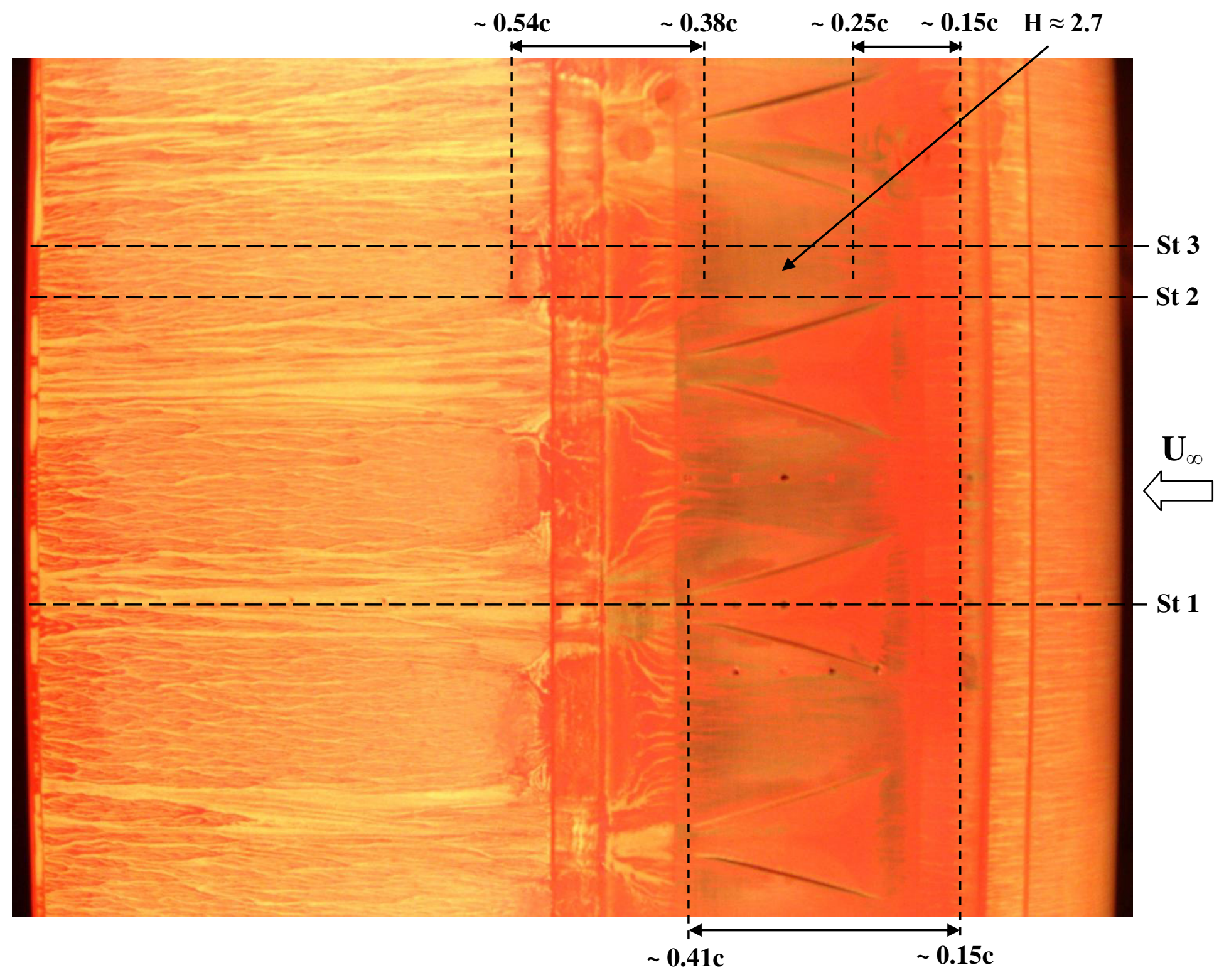

b)

Figure 9. Oil-flow patterns on model with indentations

23

American Institute of Aeronautics and Astronautics 


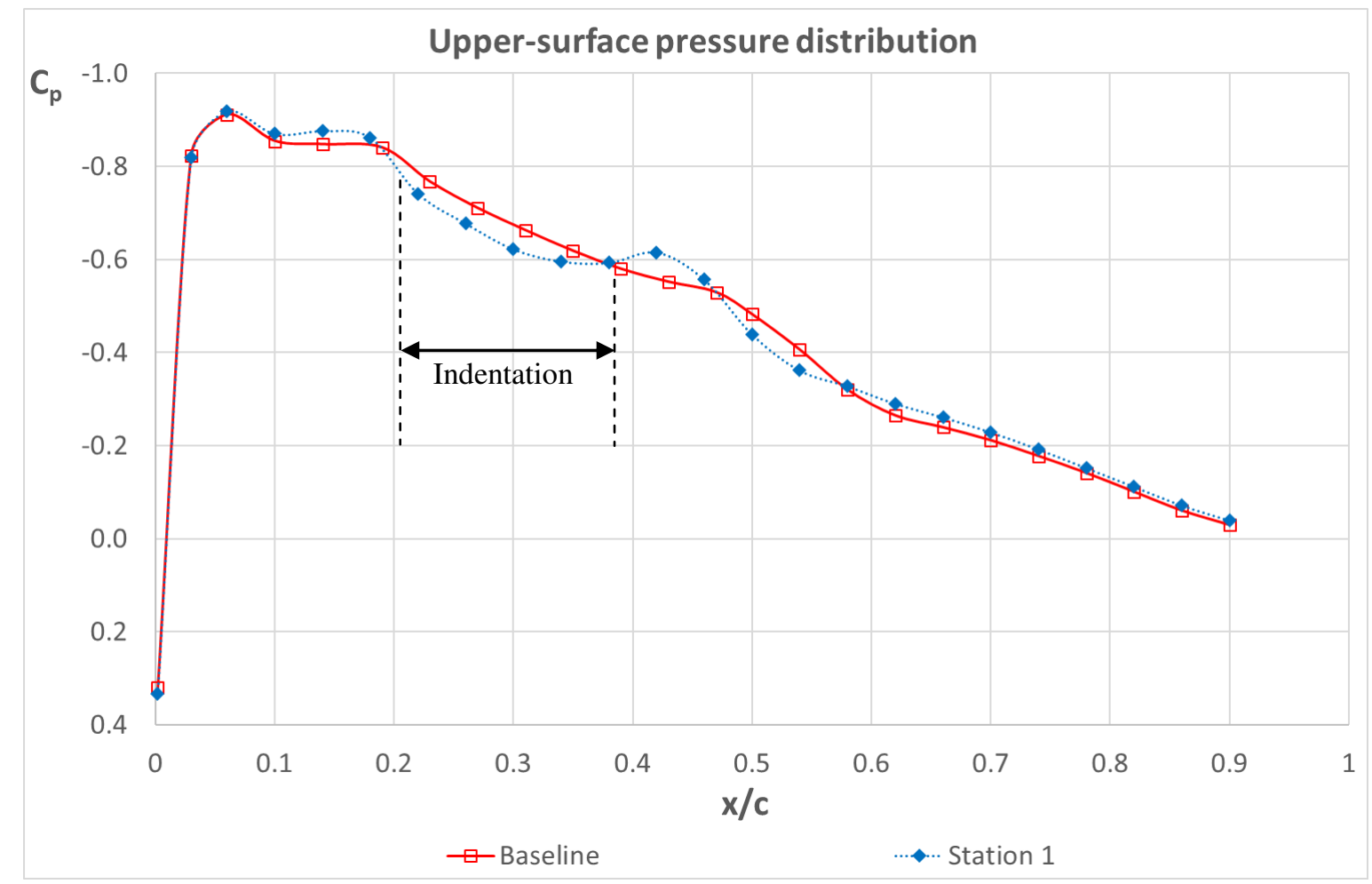

a)

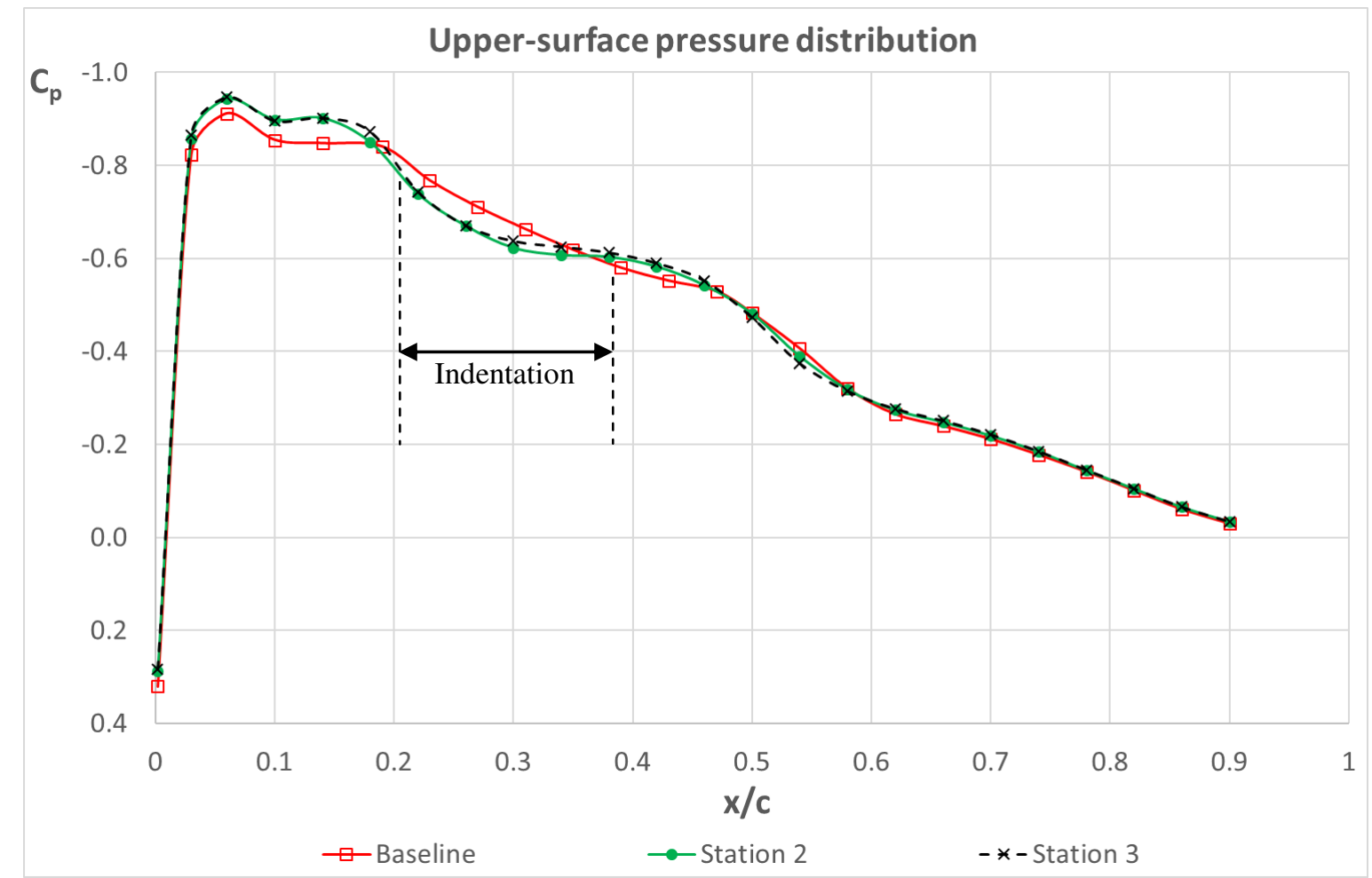

b)

Figure 10. Upper-surface pressure distributions 


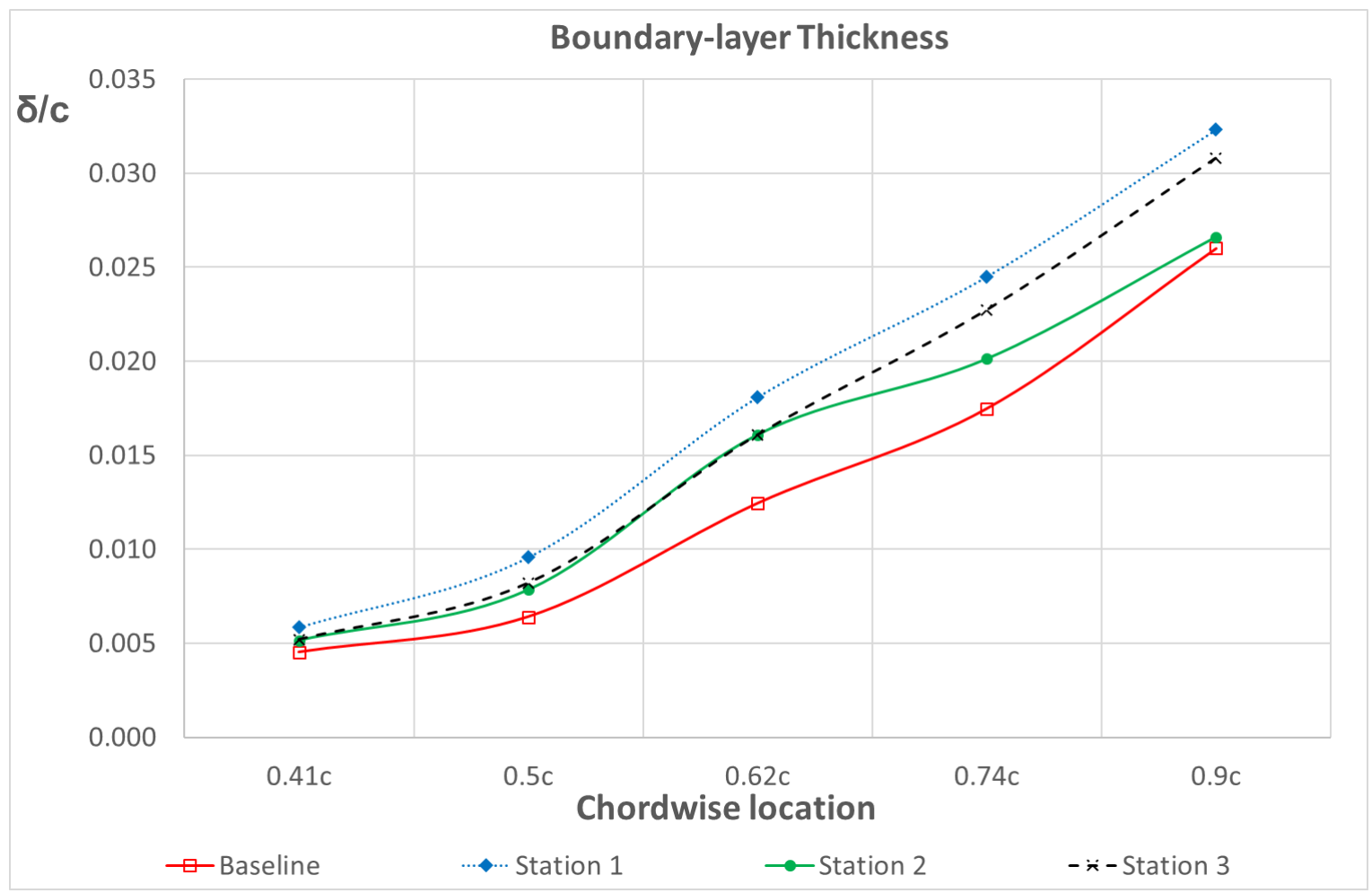

Figure 11. Development of boundary-layer thickness along the upper surface

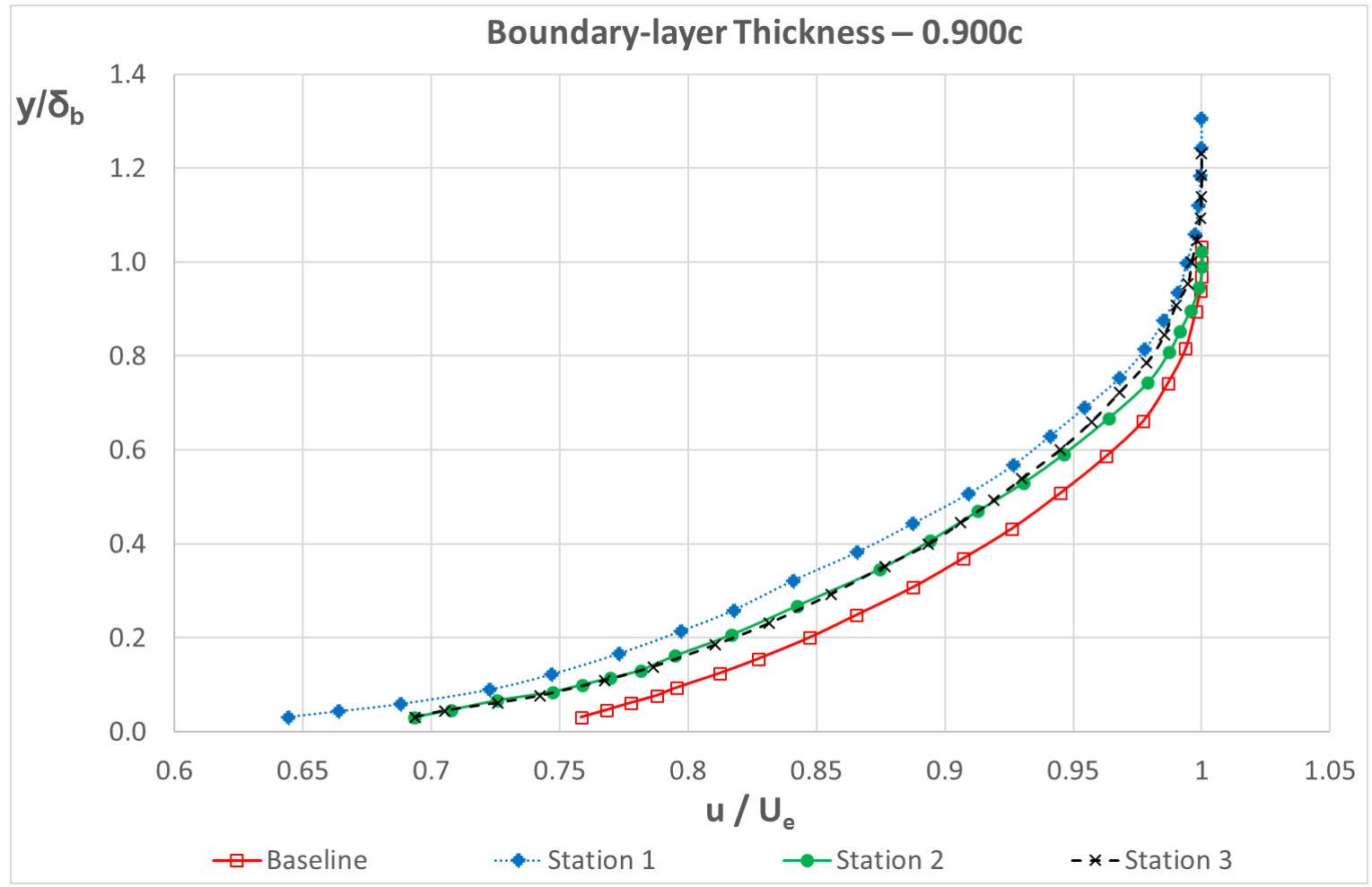

Figure 12. Boundary-layer velocity profiles at $\sim 0.900 \mathrm{c}$ 


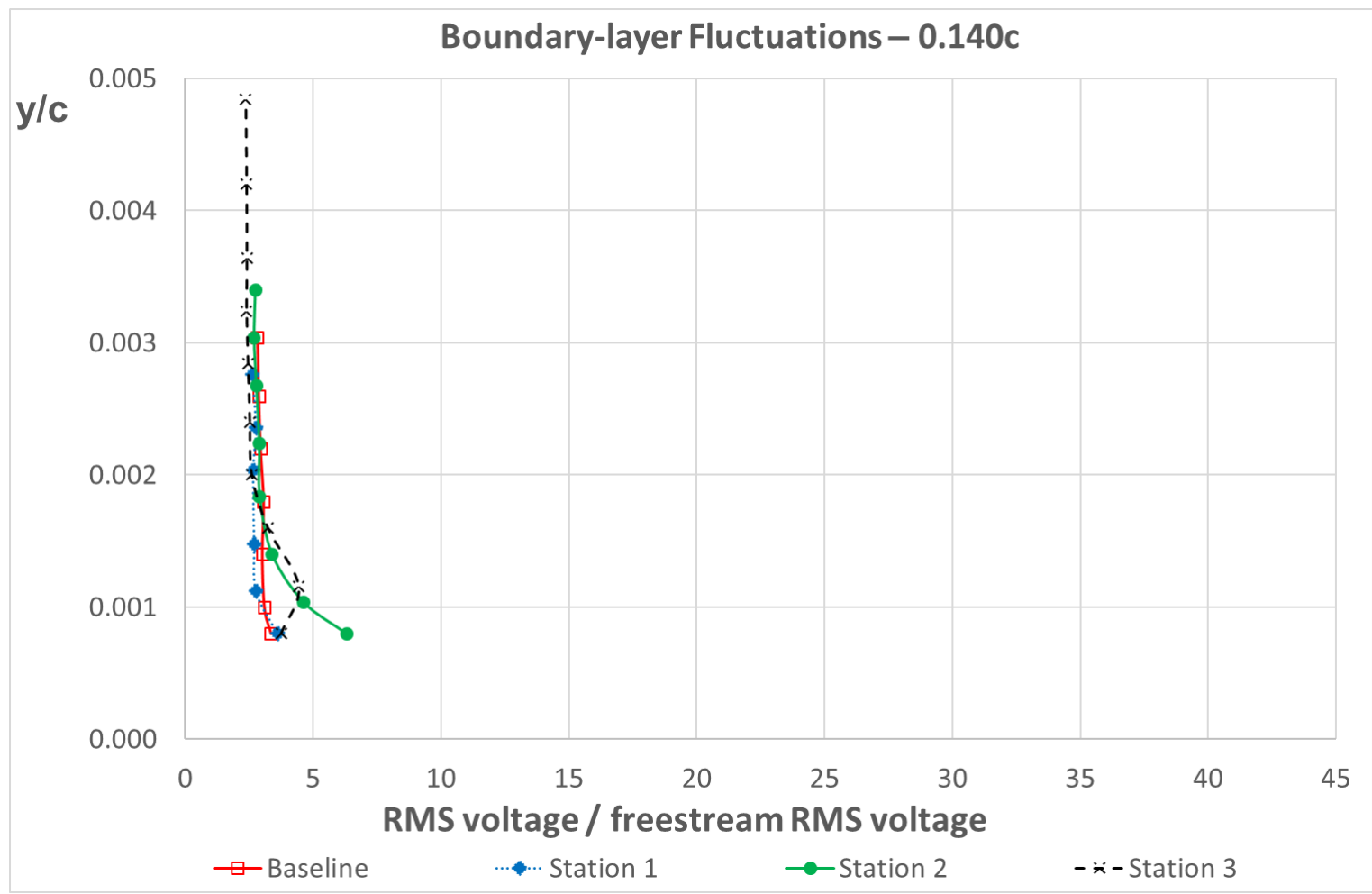

Figure 13. Boundary-layer fluctuations at $\sim 0.140 \mathrm{c}$

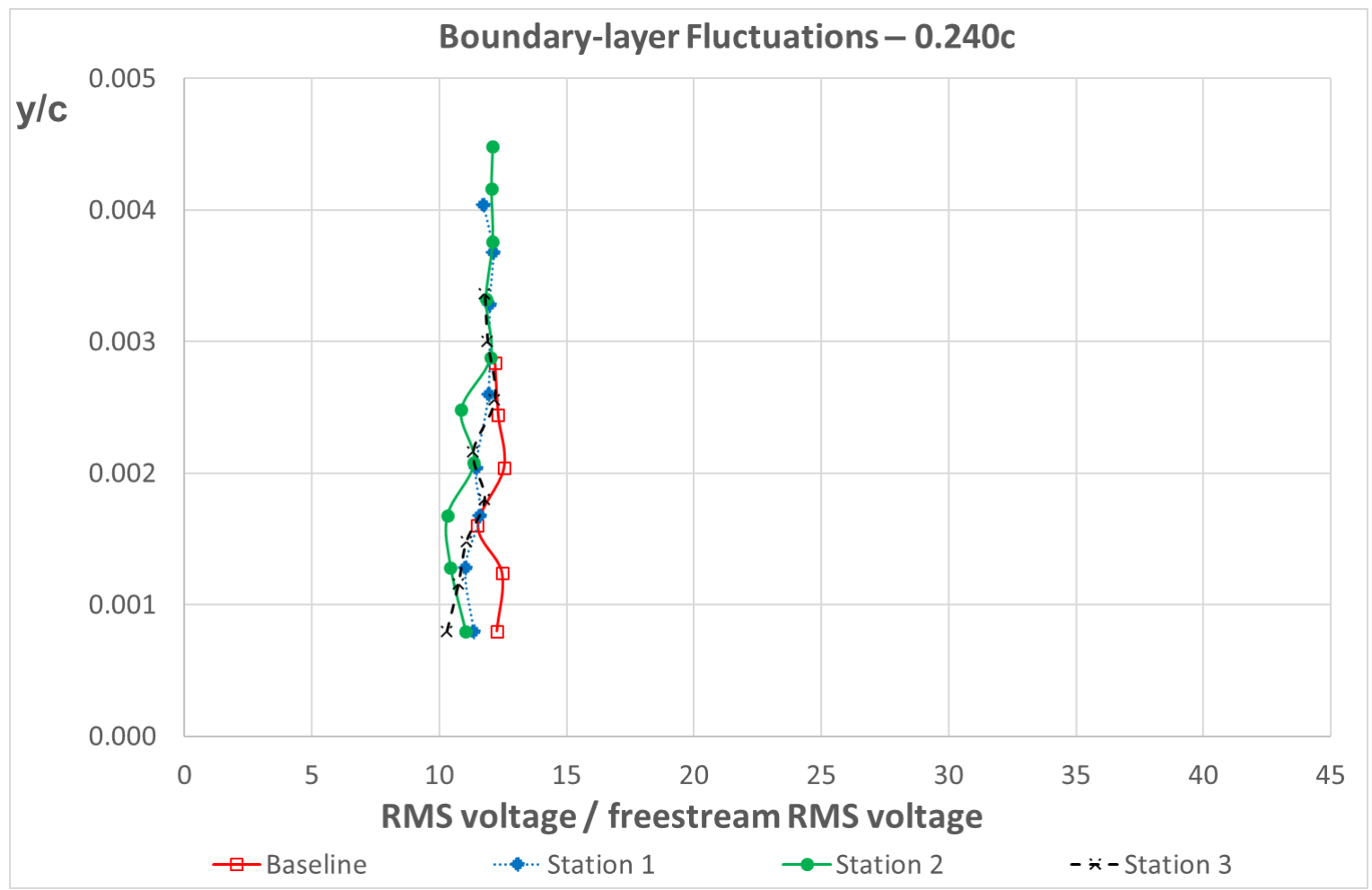

Figure 14. Boundary-layer fluctuations at $\sim 0.240 \mathrm{c}$

American Institute of Aeronautics and Astronautics 


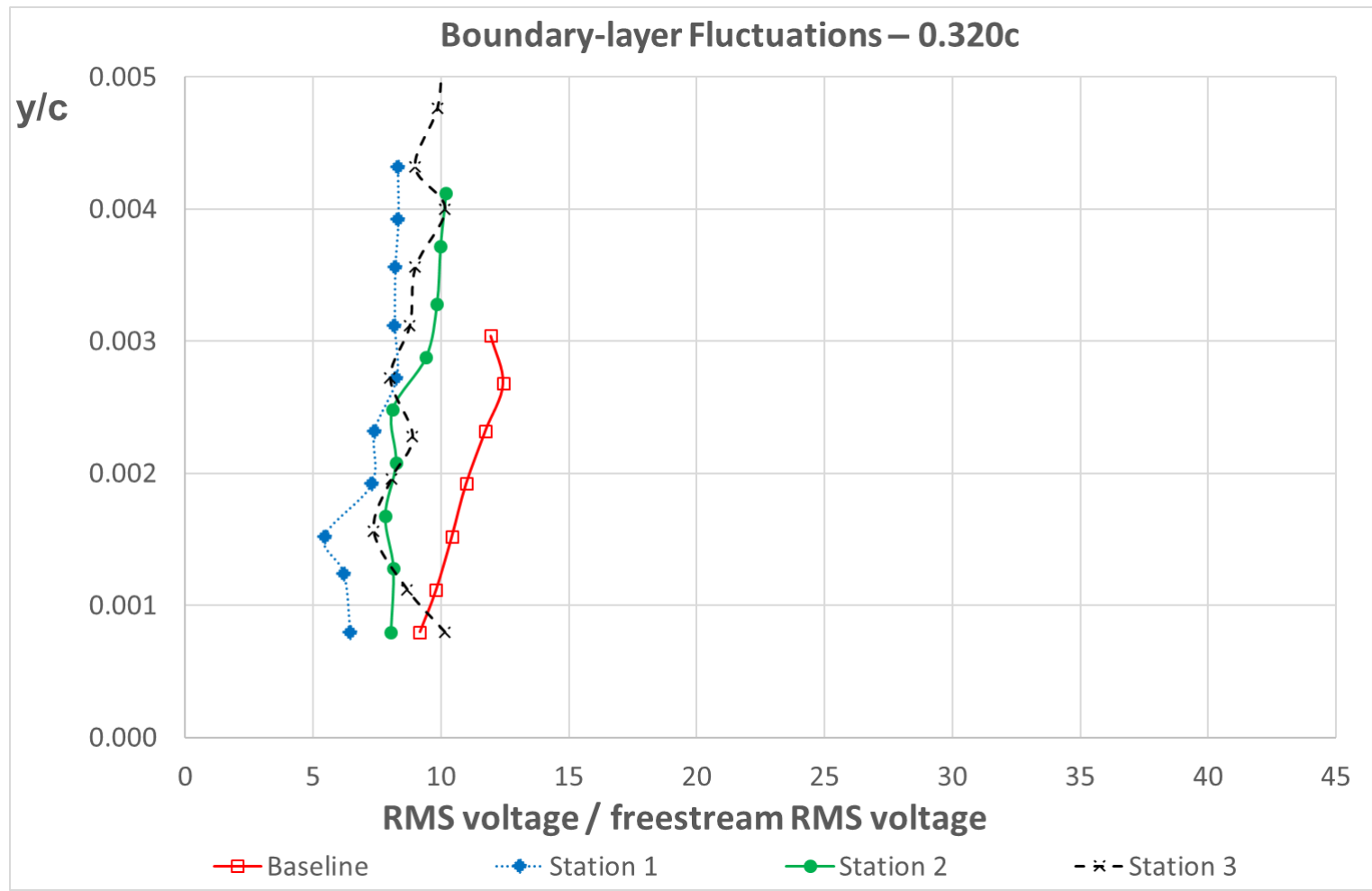

Figure 15. Boundary-layer fluctuations at $\sim 0.320 \mathrm{c}$

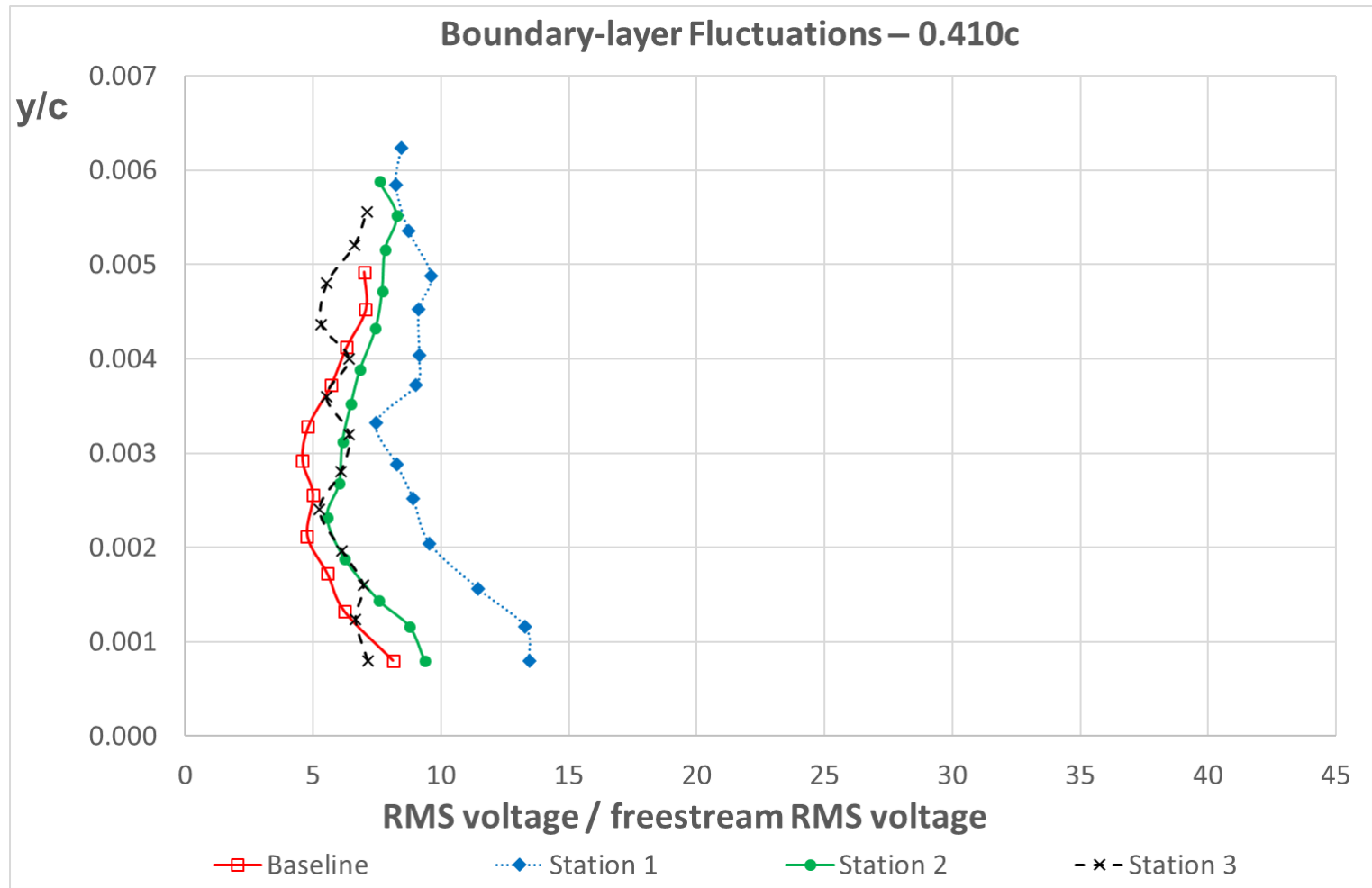

Figure 16. Boundary-layer fluctuations at $\sim 0.410 \mathrm{c}$

American Institute of Aeronautics and Astronautics 


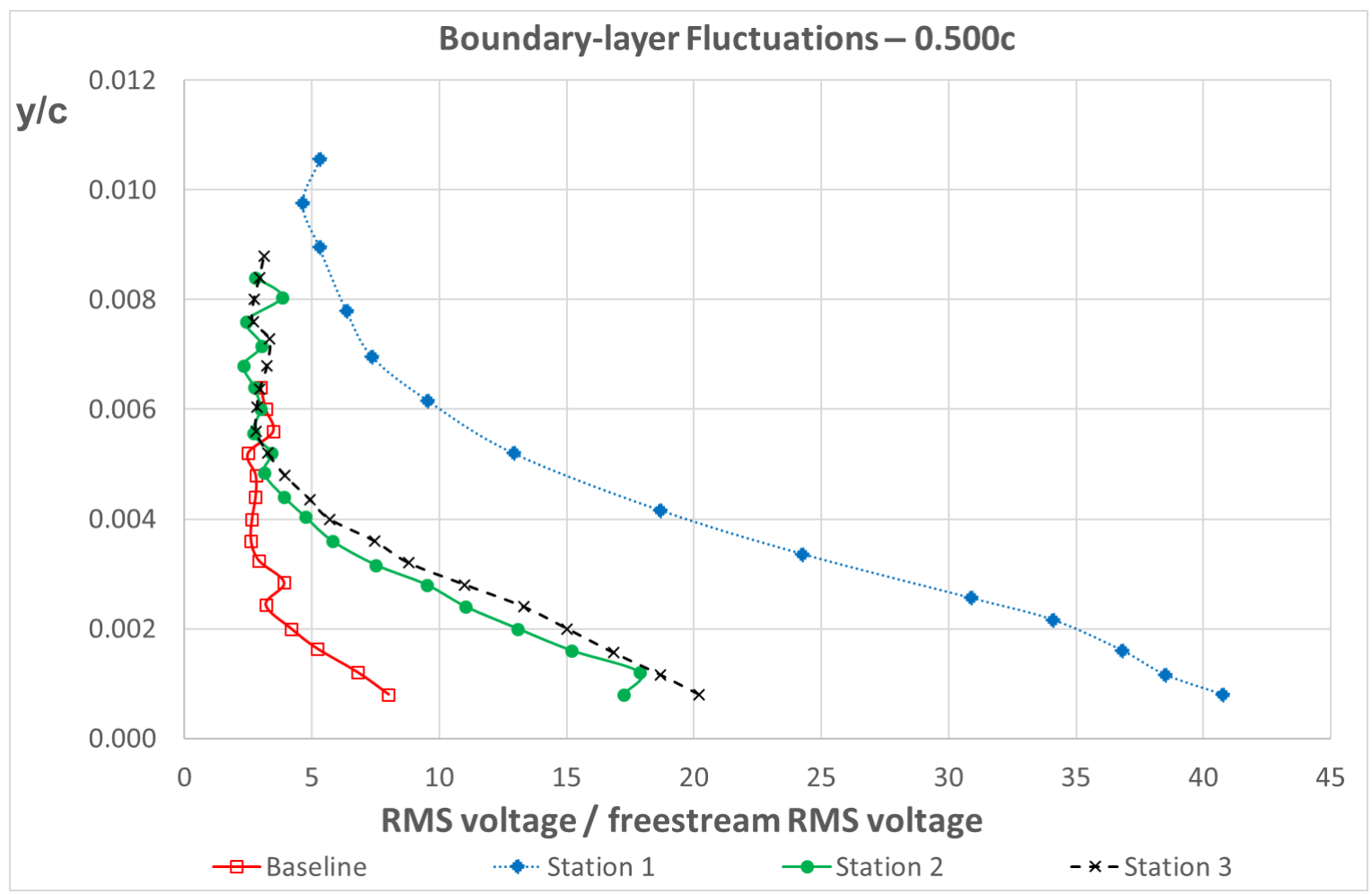

Figure 17. Boundary-layer fluctuations at $\sim 0.500 \mathrm{c}$

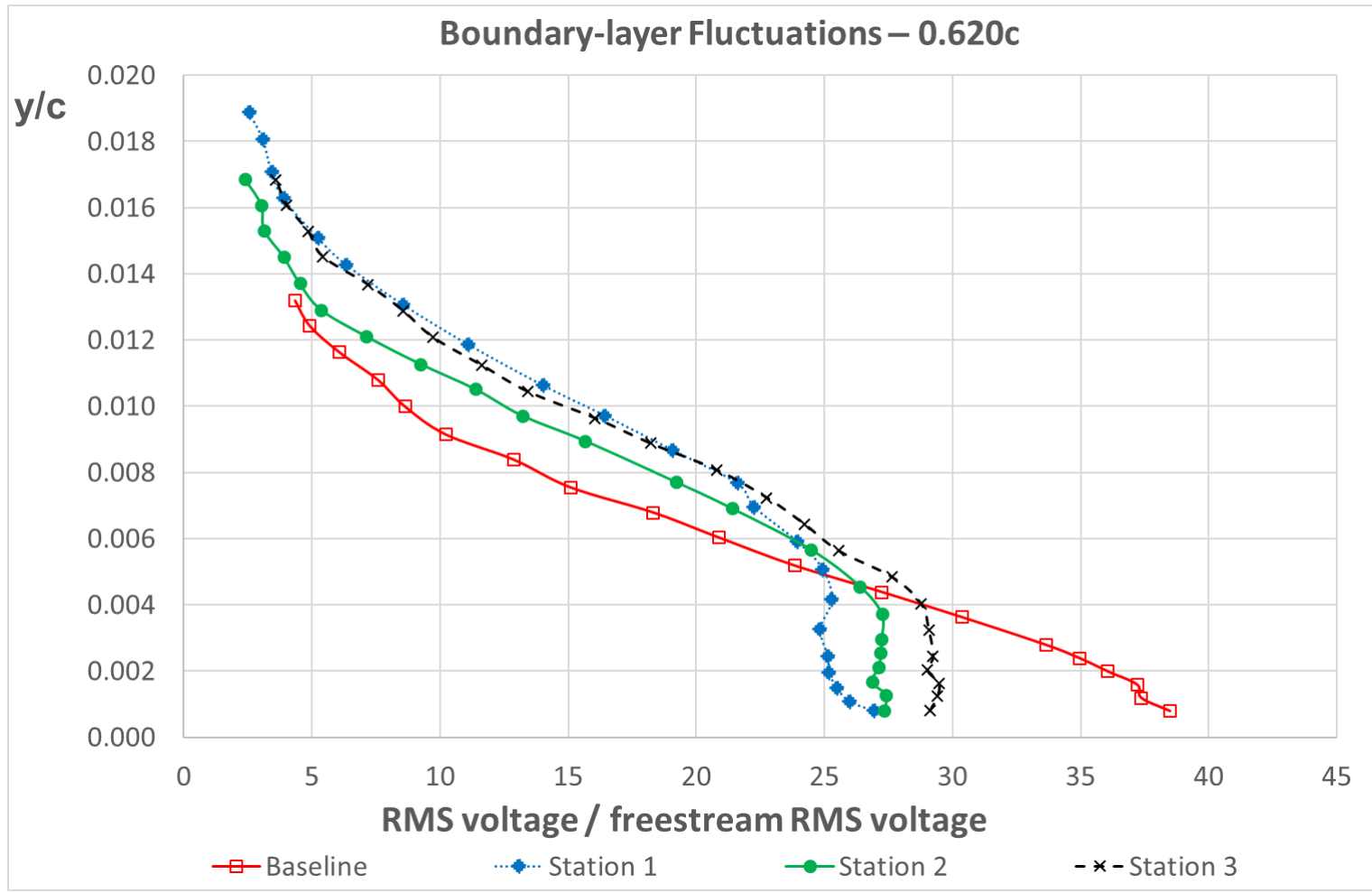

Figure 18. Boundary-layer fluctuations at $\sim 0.620 \mathrm{c}$

American Institute of Aeronautics and Astronautics 


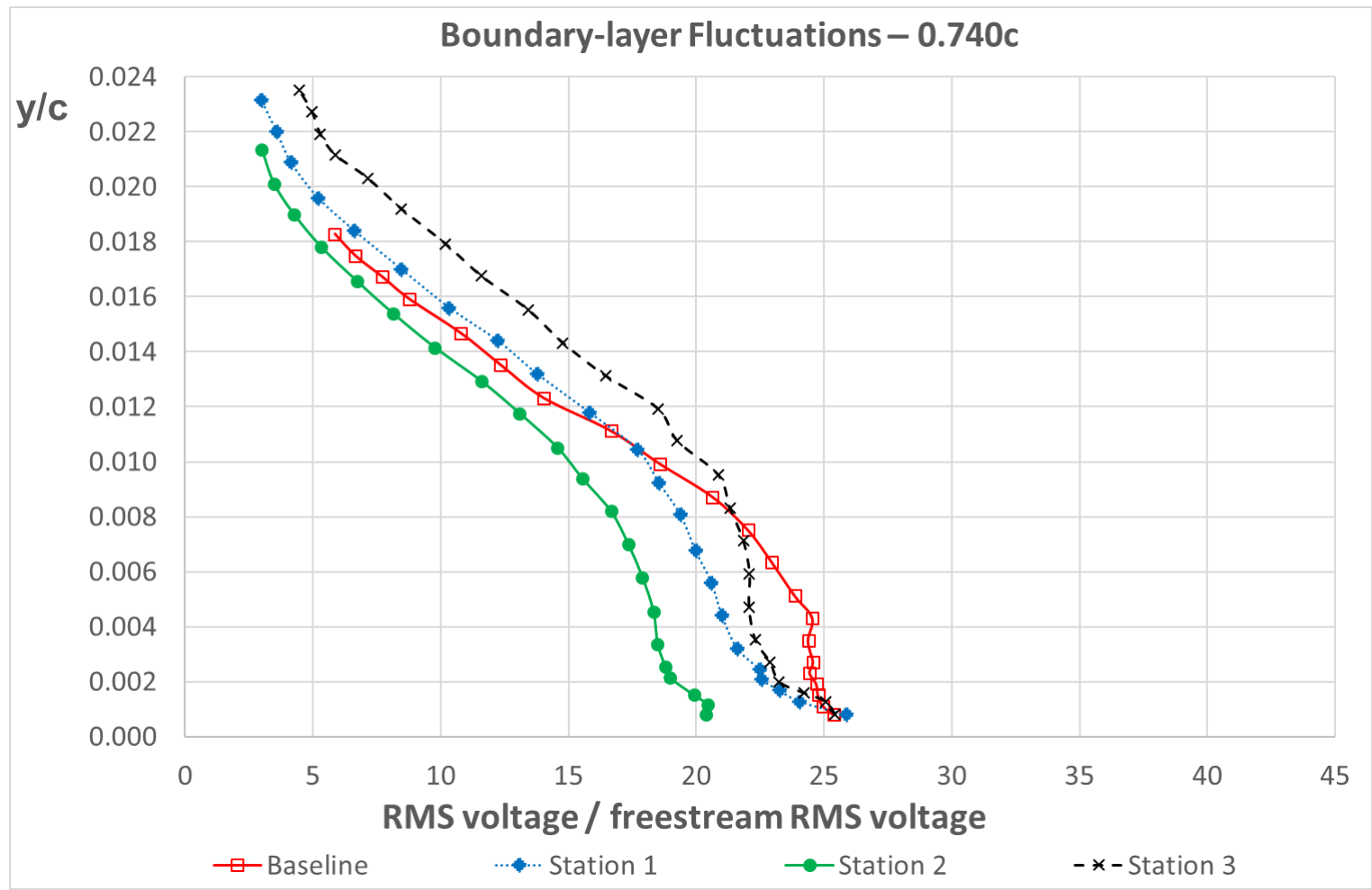

Figure 19. Boundary-layer fluctuations at $\sim 0.740 \mathrm{c}$

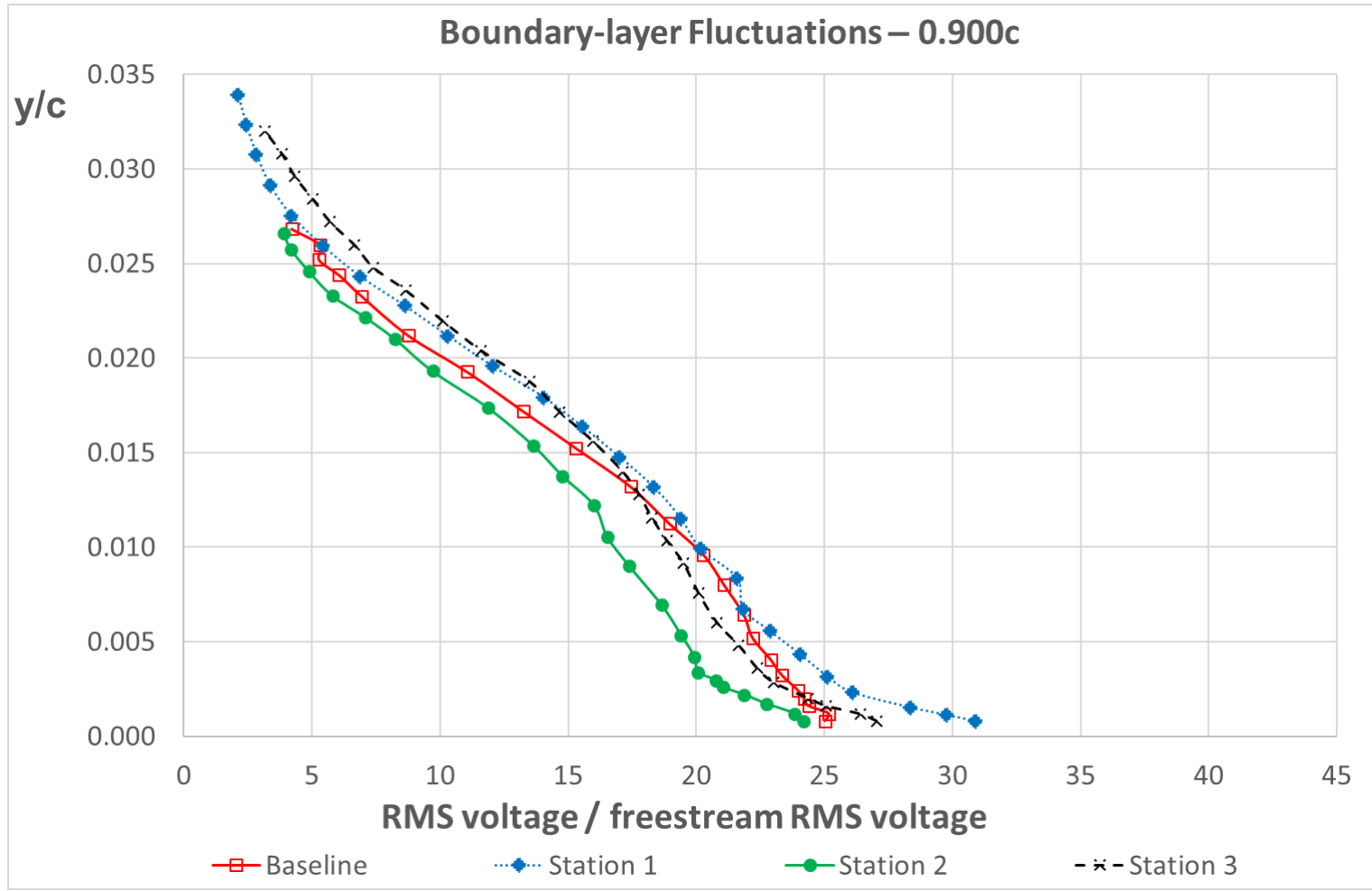

Figure 20. Boundary-layer fluctuations at $\sim 0.900 \mathrm{c}$

American Institute of Aeronautics and Astronautics 

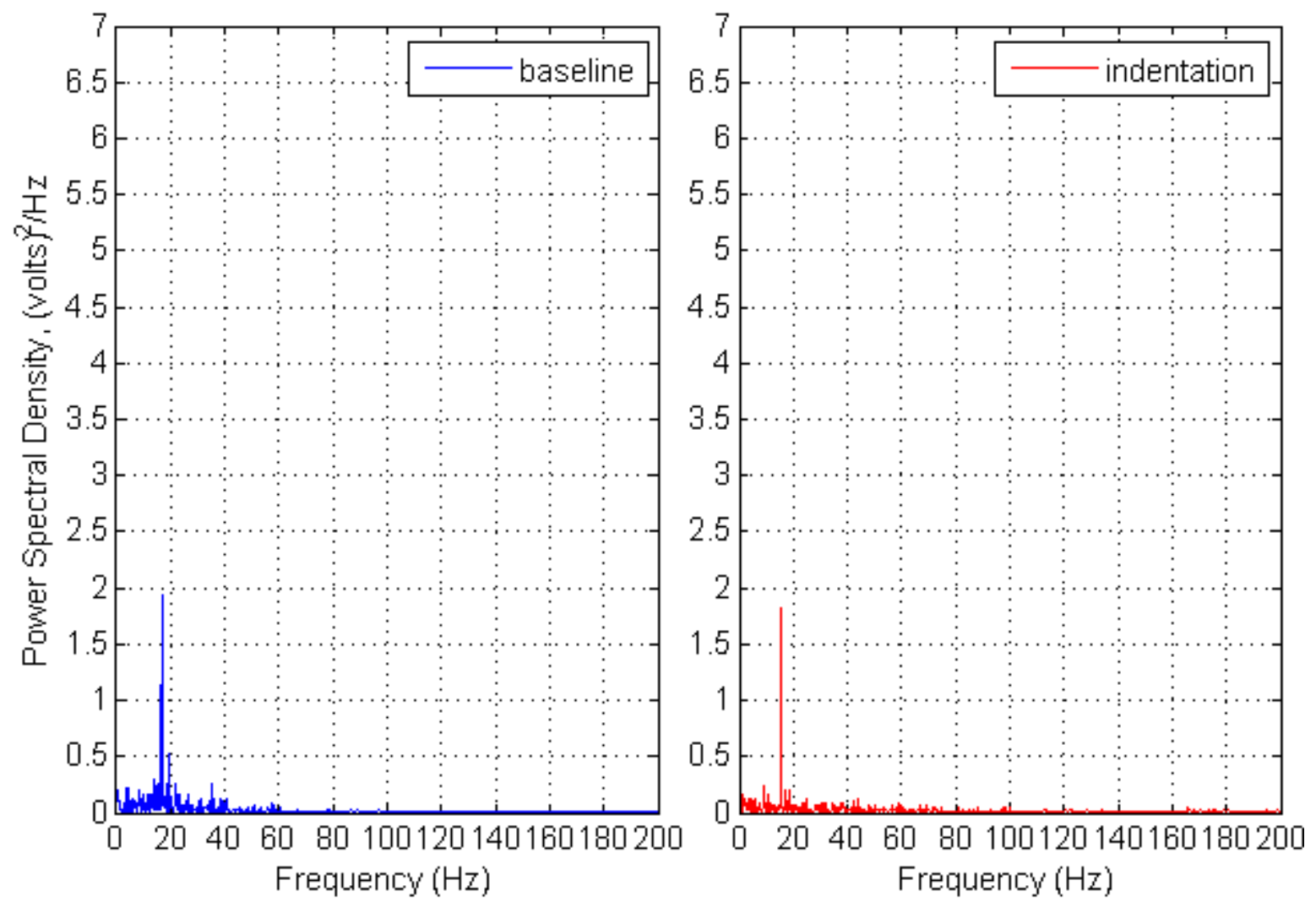

Figure 21. Power spectral analysis at $y / c \approx 0.0008, \sim 0.410 c$, and spanwise Station 1
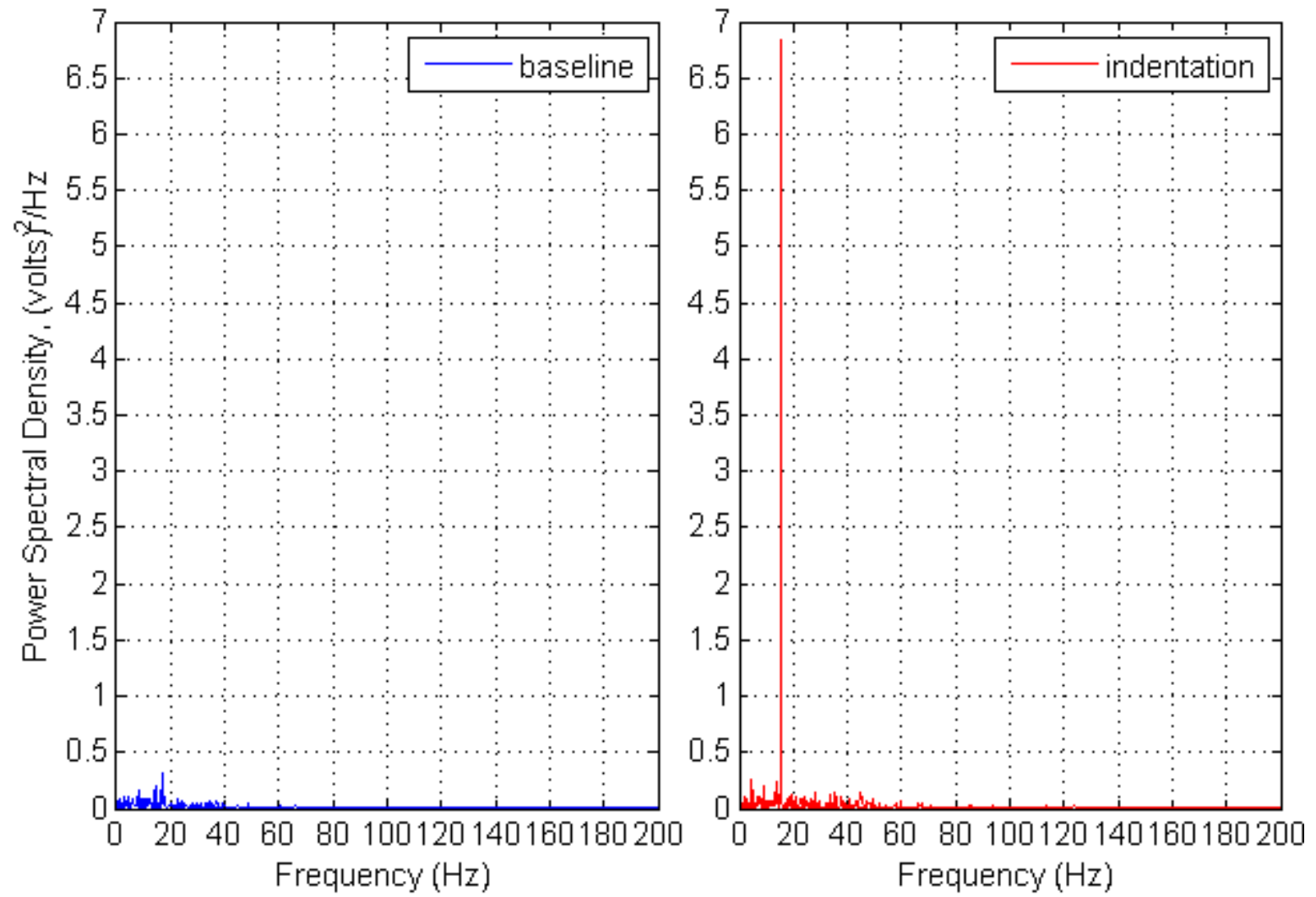

Figure 22. Power spectral analysis at $y / c \approx 0.0016, \sim 0.410 c$, and spanwise Station 1

30

American Institute of Aeronautics and Astronautics 

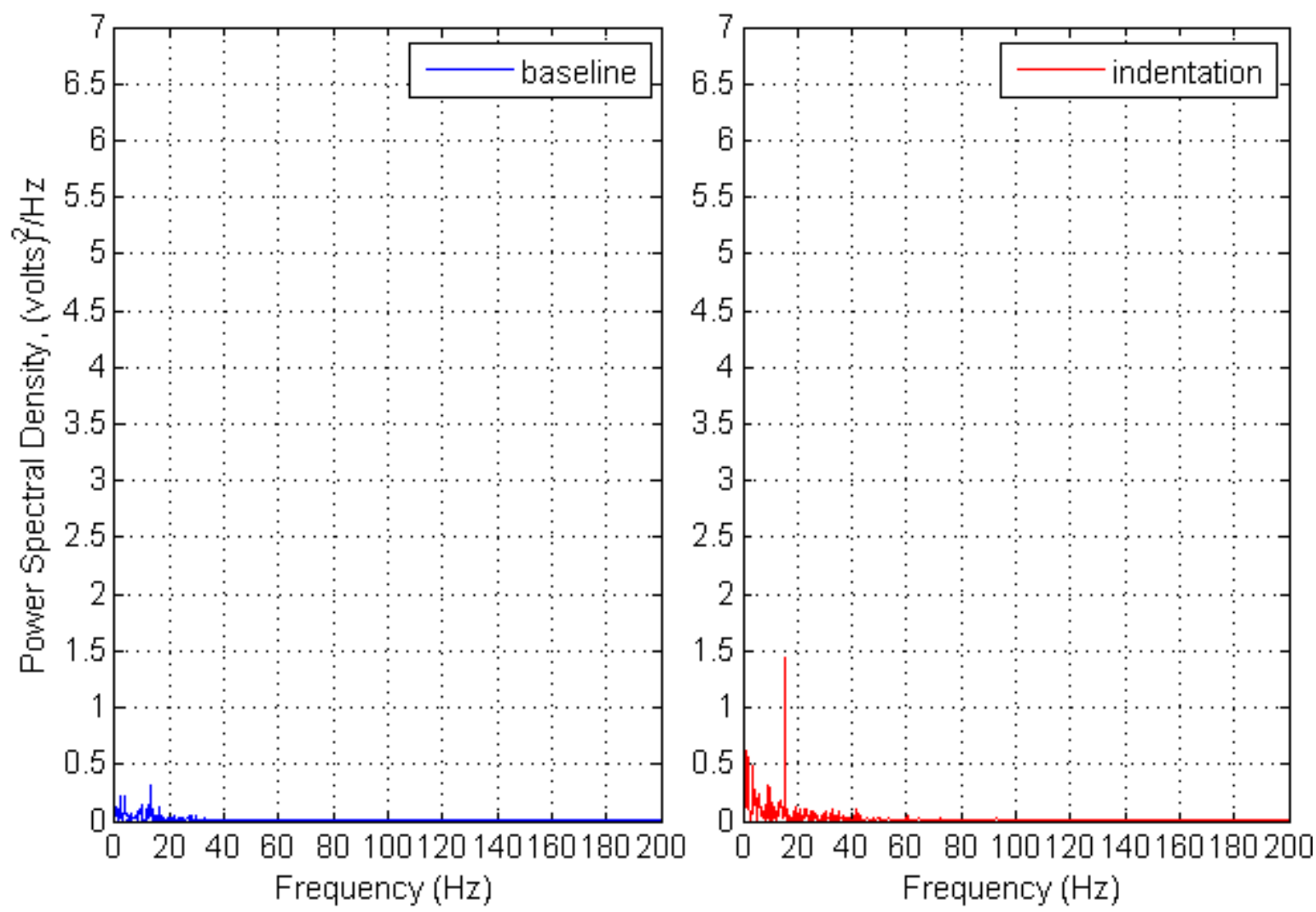

Figure 23. Power spectral analysis at $y / c \approx 0.0025, \sim 0.410 c$, and spanwise Station 1
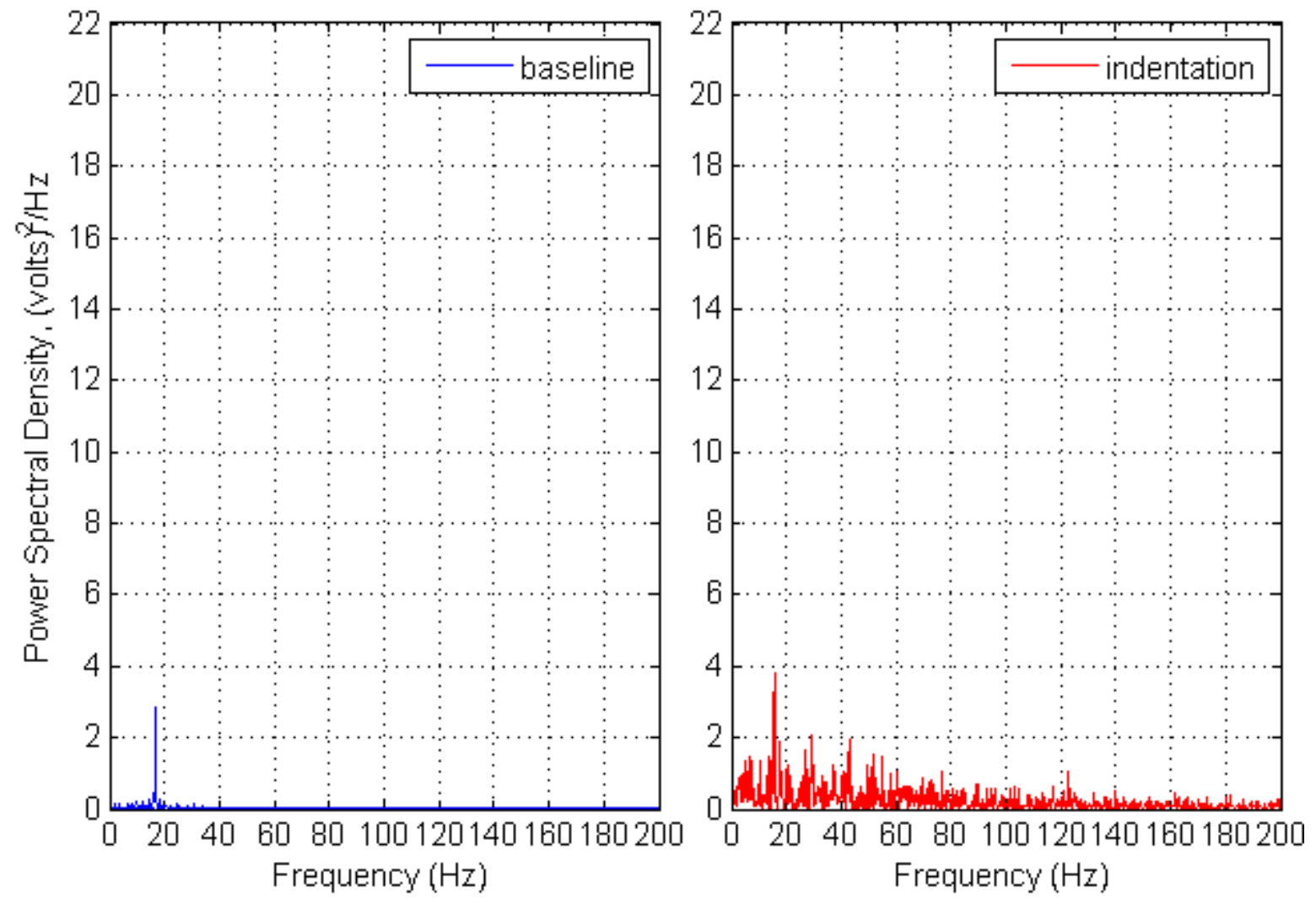

Figure 24. Power spectral analysis at $y / c \approx 0.0008, \sim 0.500 c$, and spanwise Station 1

31

American Institute of Aeronautics and Astronautics 

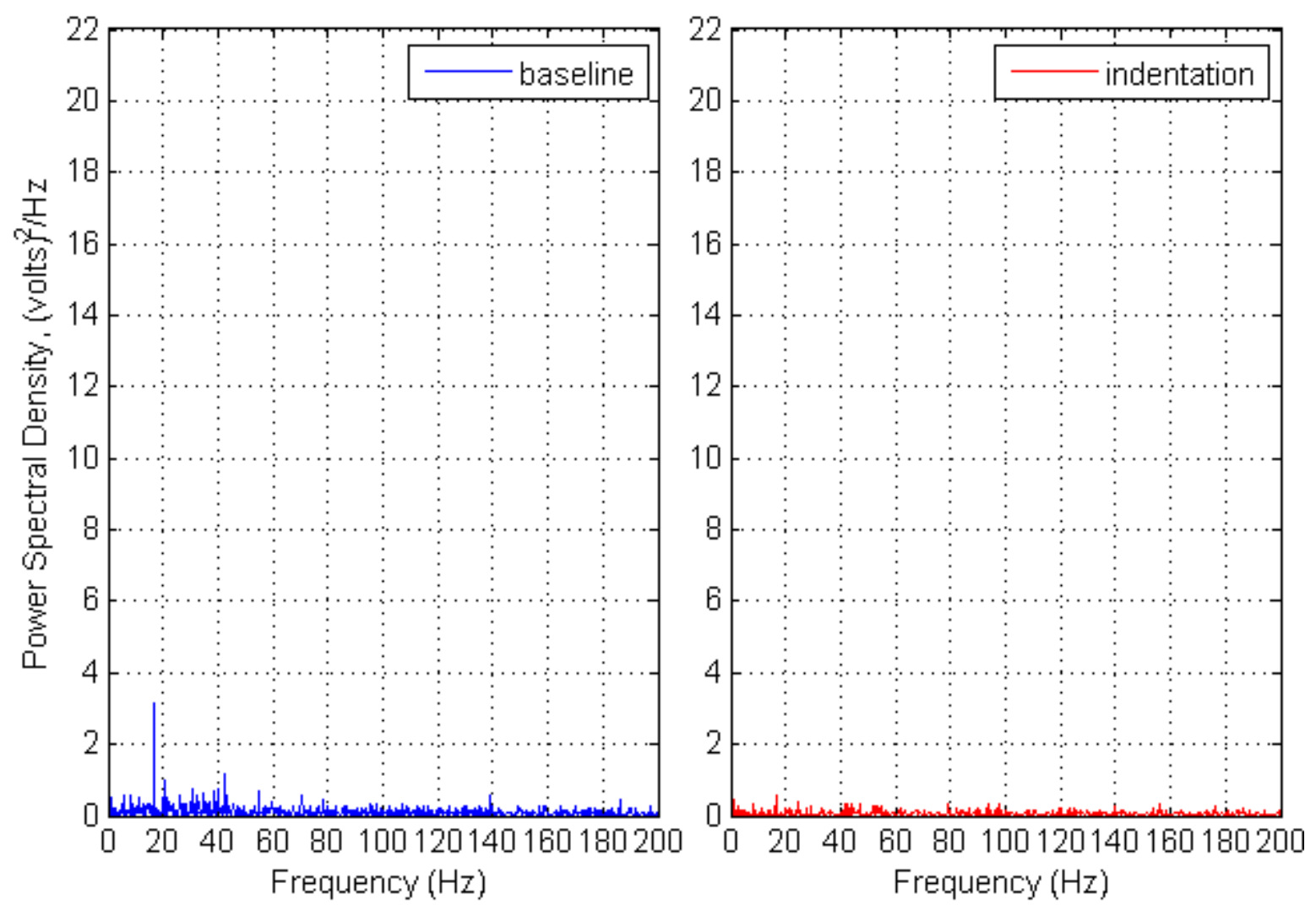

Figure 25. Power spectral analysis at $y / c \approx 0.0008, \sim 0.620 c$, and spanwise Station 3
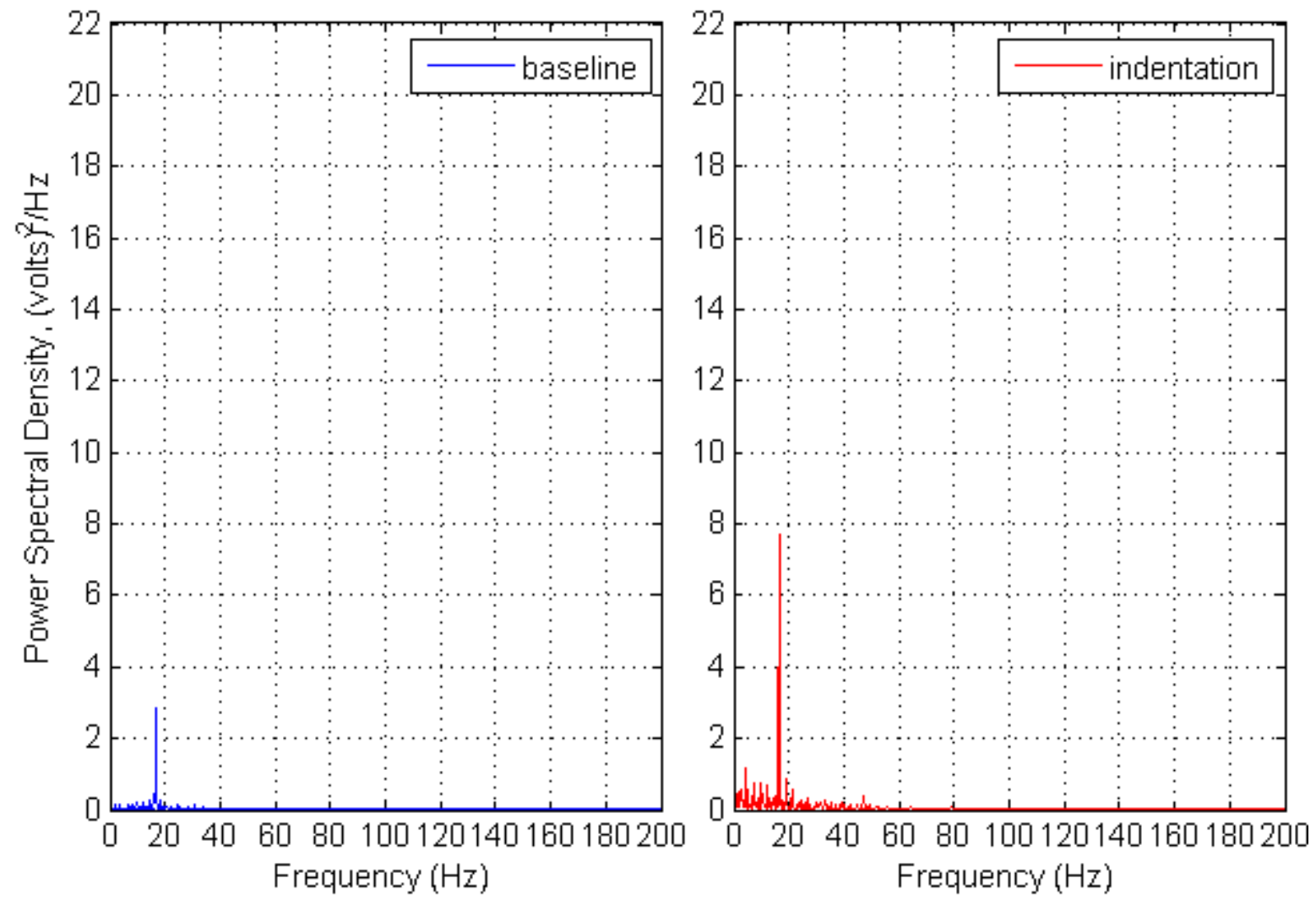

Figure 26. Power spectral analysis at $y / c \approx 0.0008, \sim 0.500 c$, and spanwise Station 2

32

American Institute of Aeronautics and Astronautics 

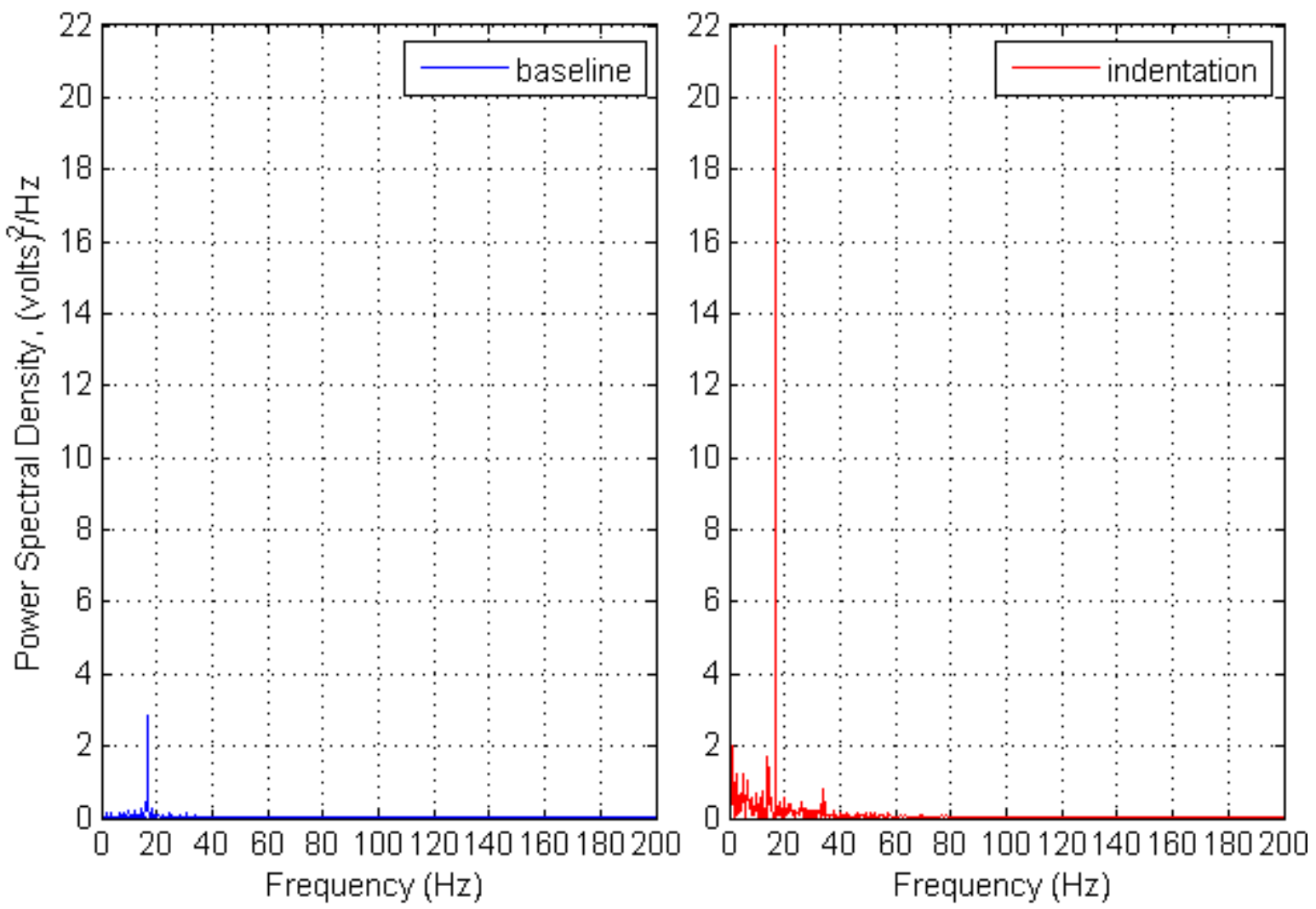

Figure 27. Power spectral analysis at $y / c \approx 0.0008, \sim 0.500 \mathrm{c}$ and spanwise Station 3 


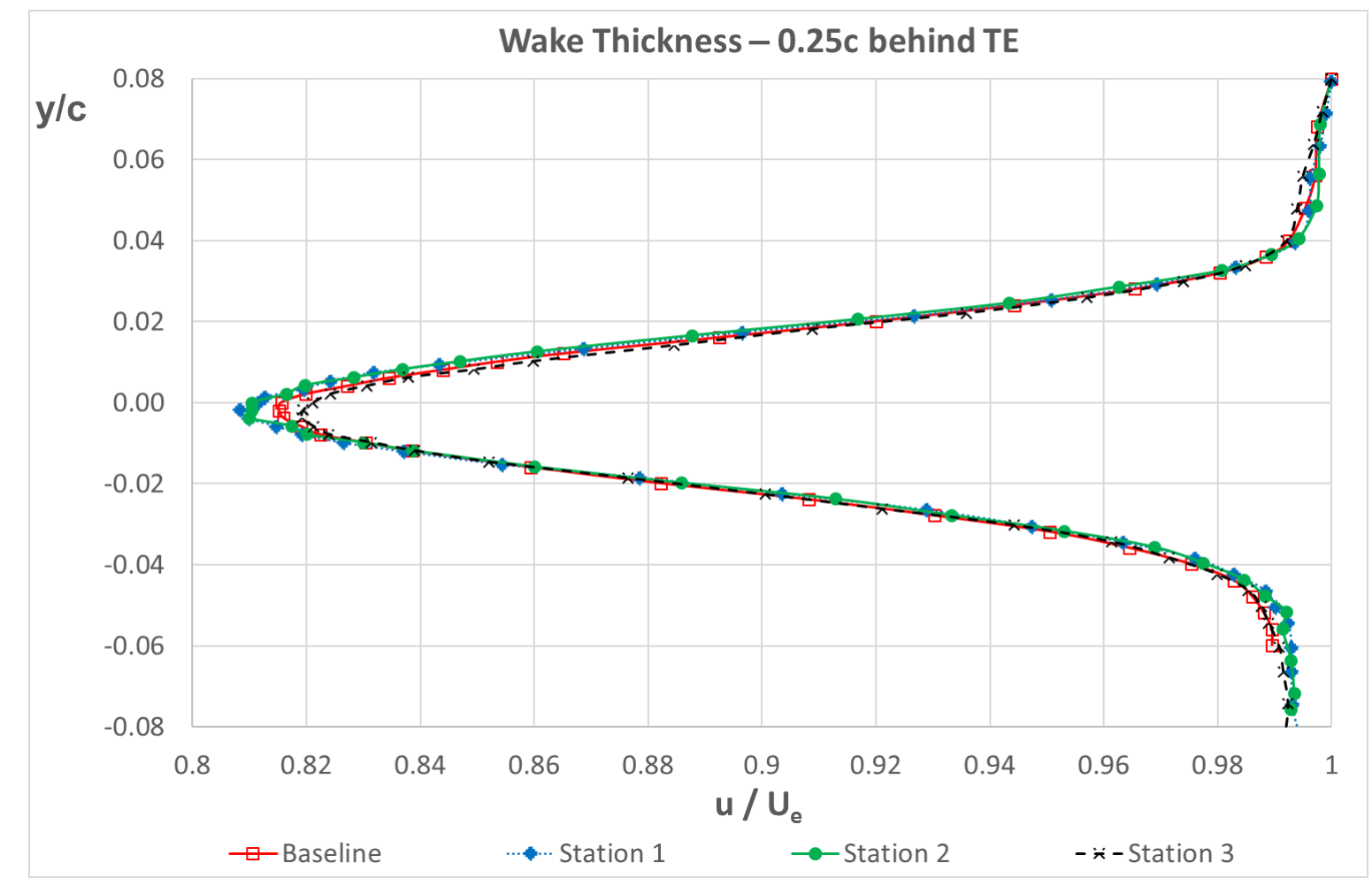

a)

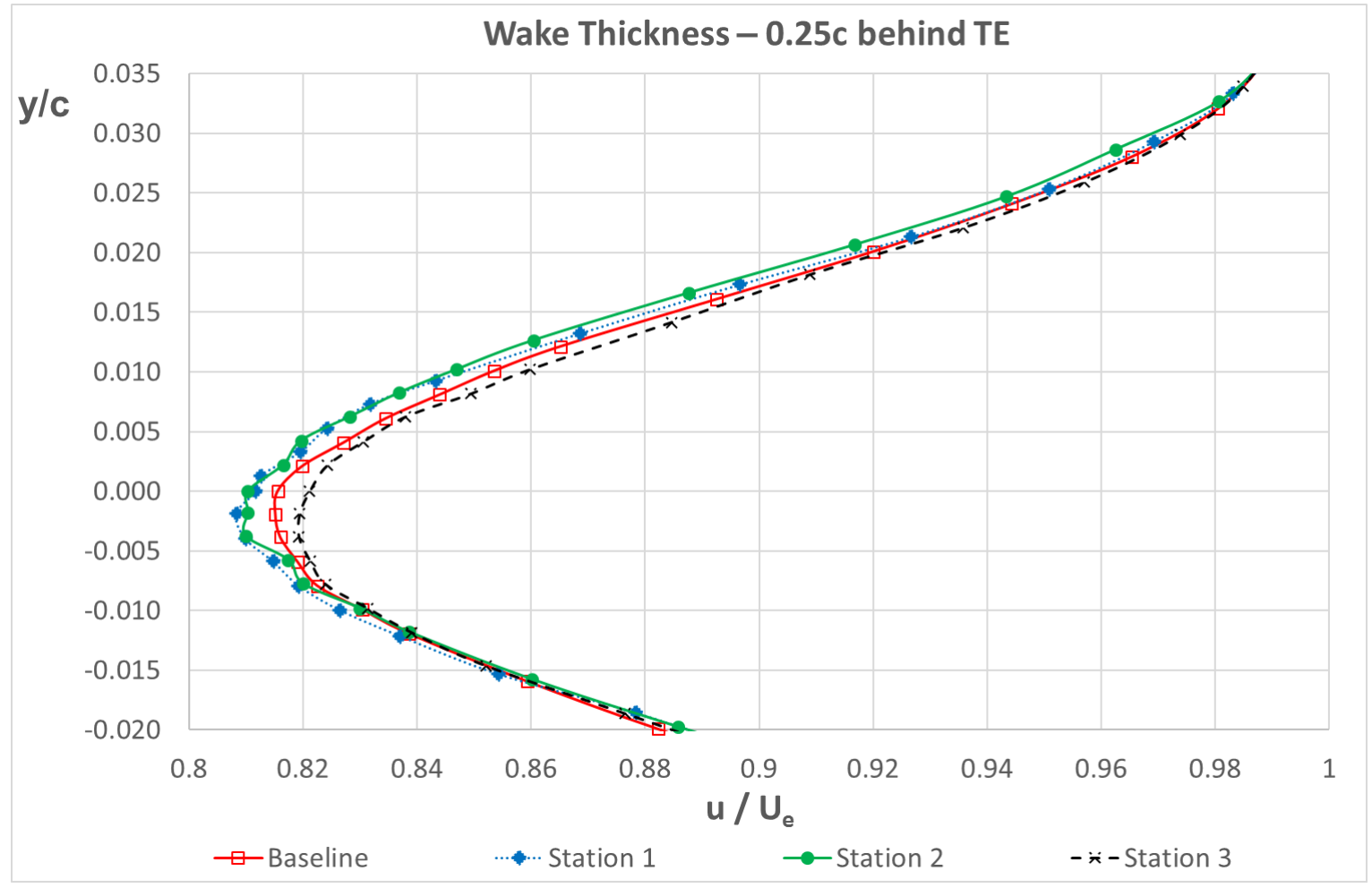

b)

Figure 28. Wake-velocity profiles at $\sim 0.250 \mathrm{c}$ downstream of aerofoil trailing edge 


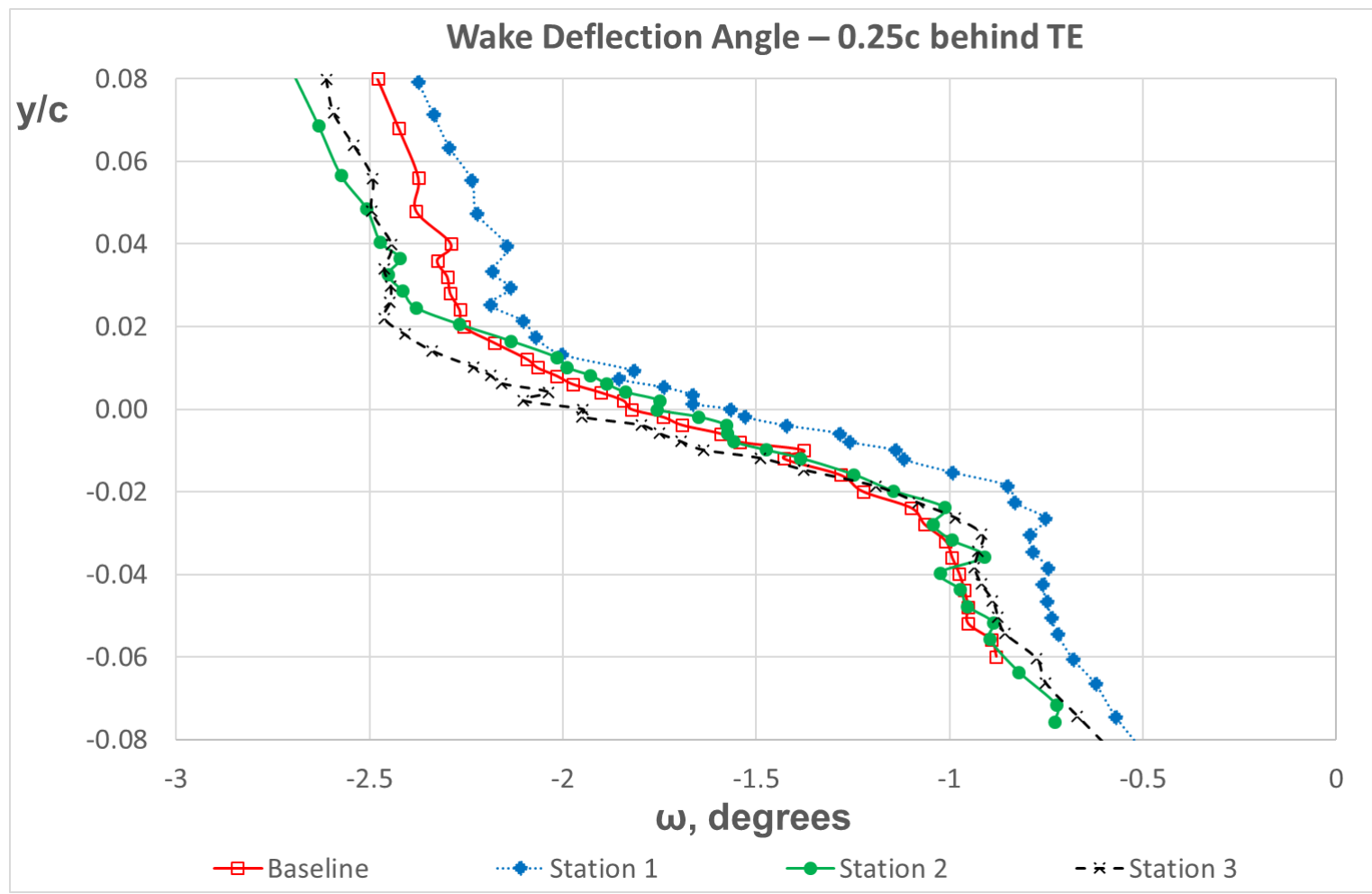

Figure 29. Wake-deflection angles at $\sim 0.250 \mathrm{c}$ downstream of aerofoil trailing edge

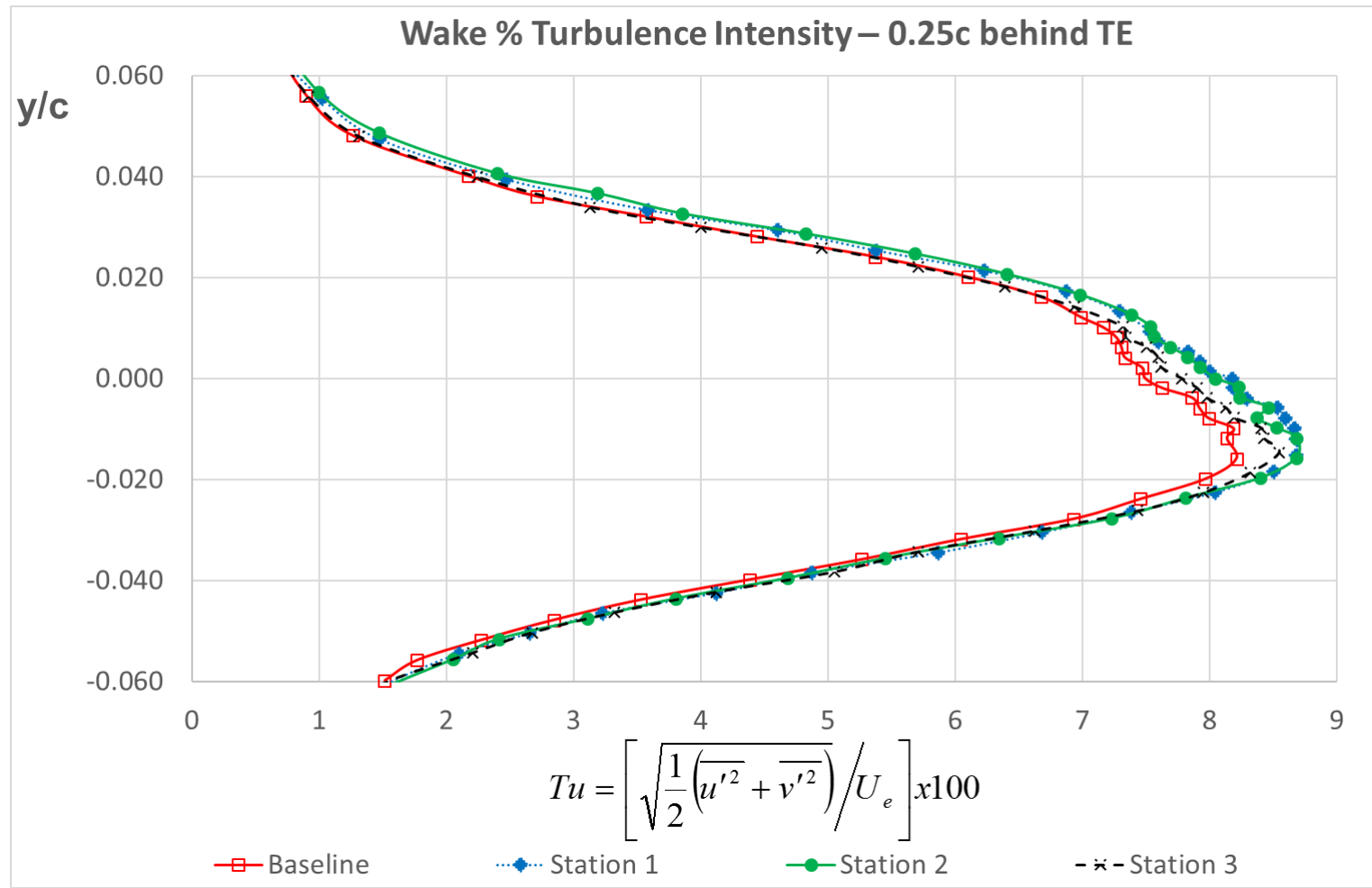

Figure 30. Percentage turbulence intensity, Tu, at $\sim 0.250 \mathrm{c}$ downstream of aerofoil trailing edge 


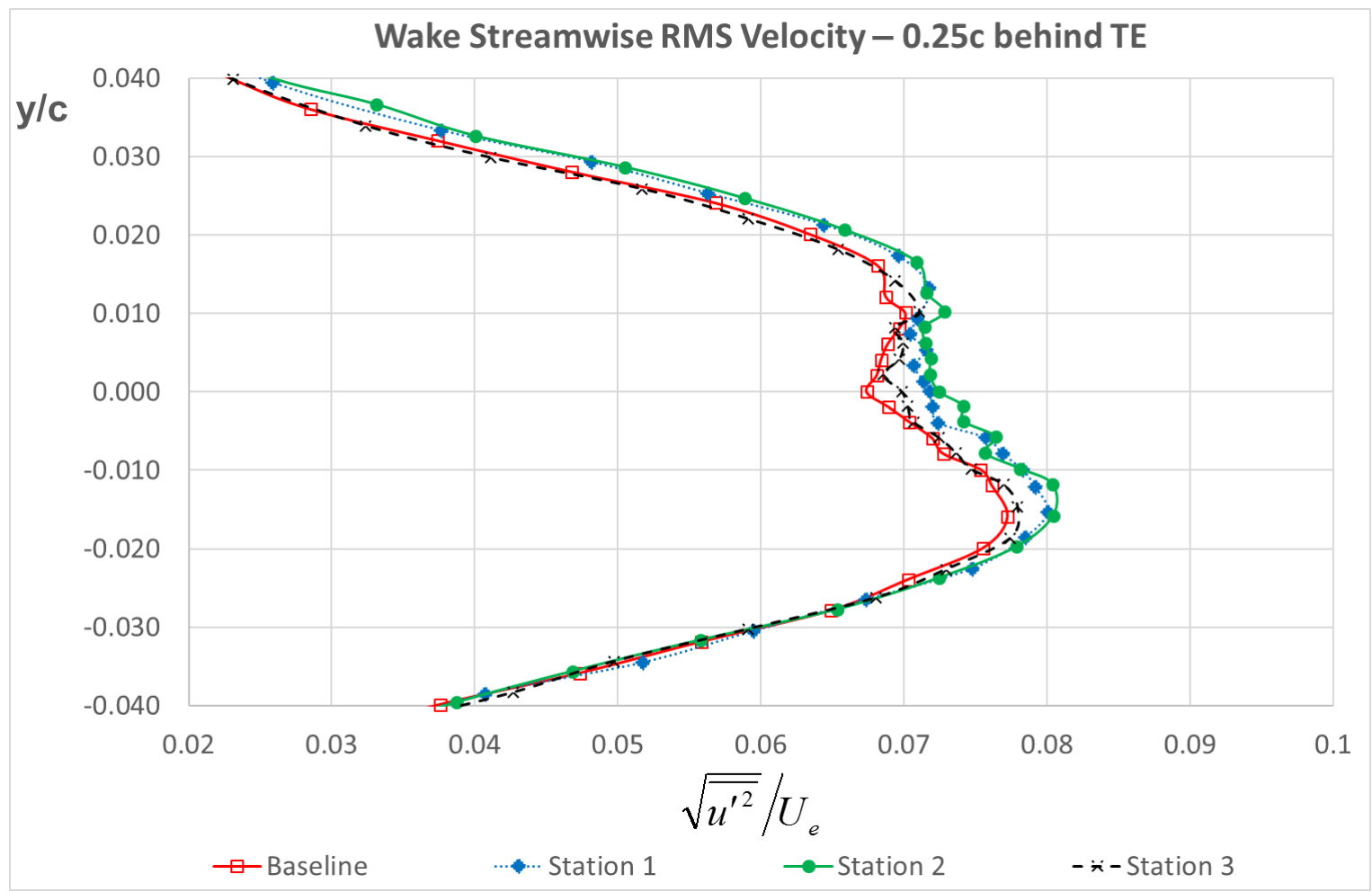

Figure 31. u-component RMS-velocity profiles at $\sim 0.250 \mathrm{c}$ downstream of aerofoil trailing edge

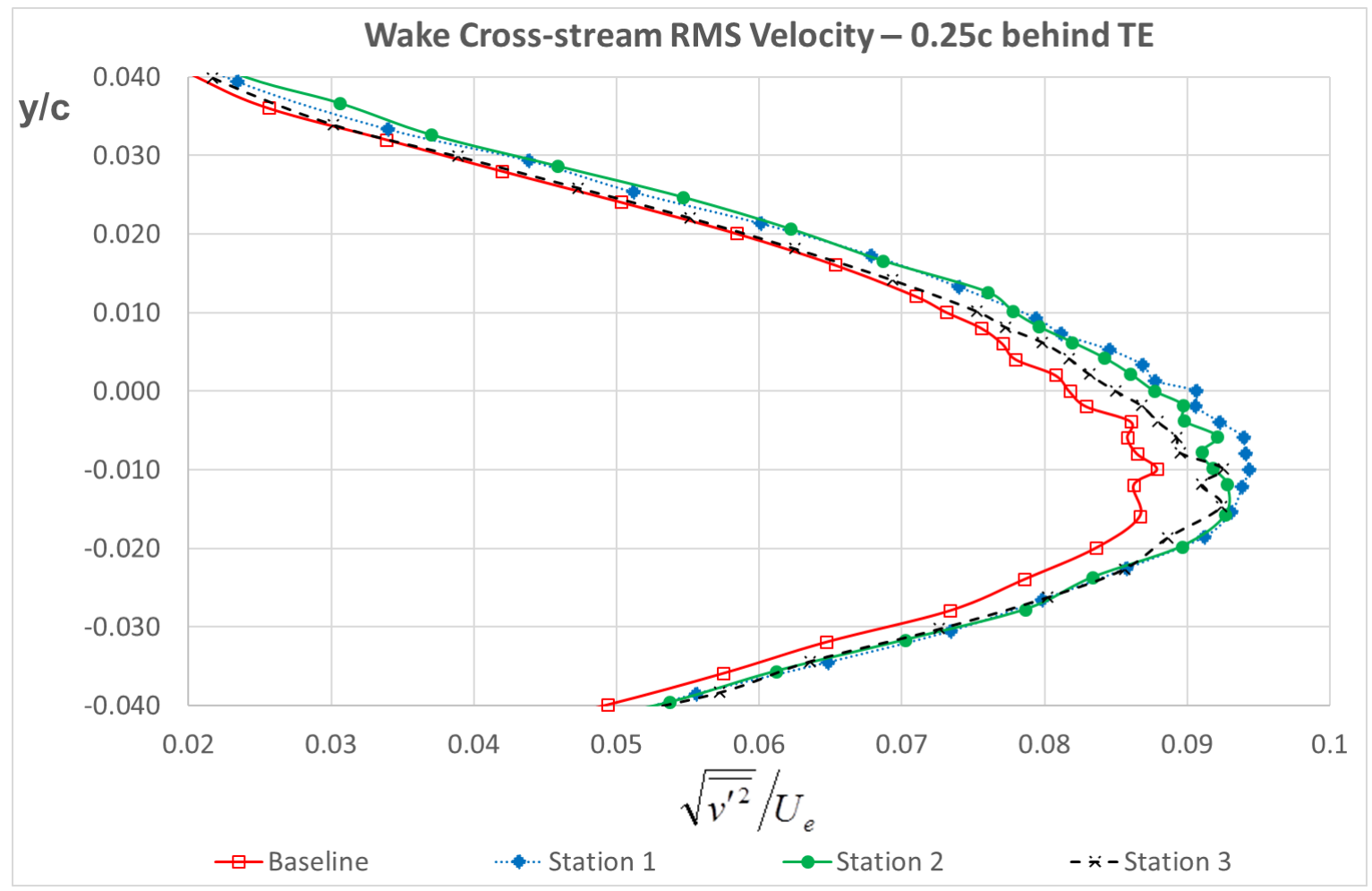

Figure 32. v-component RMS-velocity profiles at $\sim 0.250 \mathrm{c}$ downstream of aerofoil trailing edge 

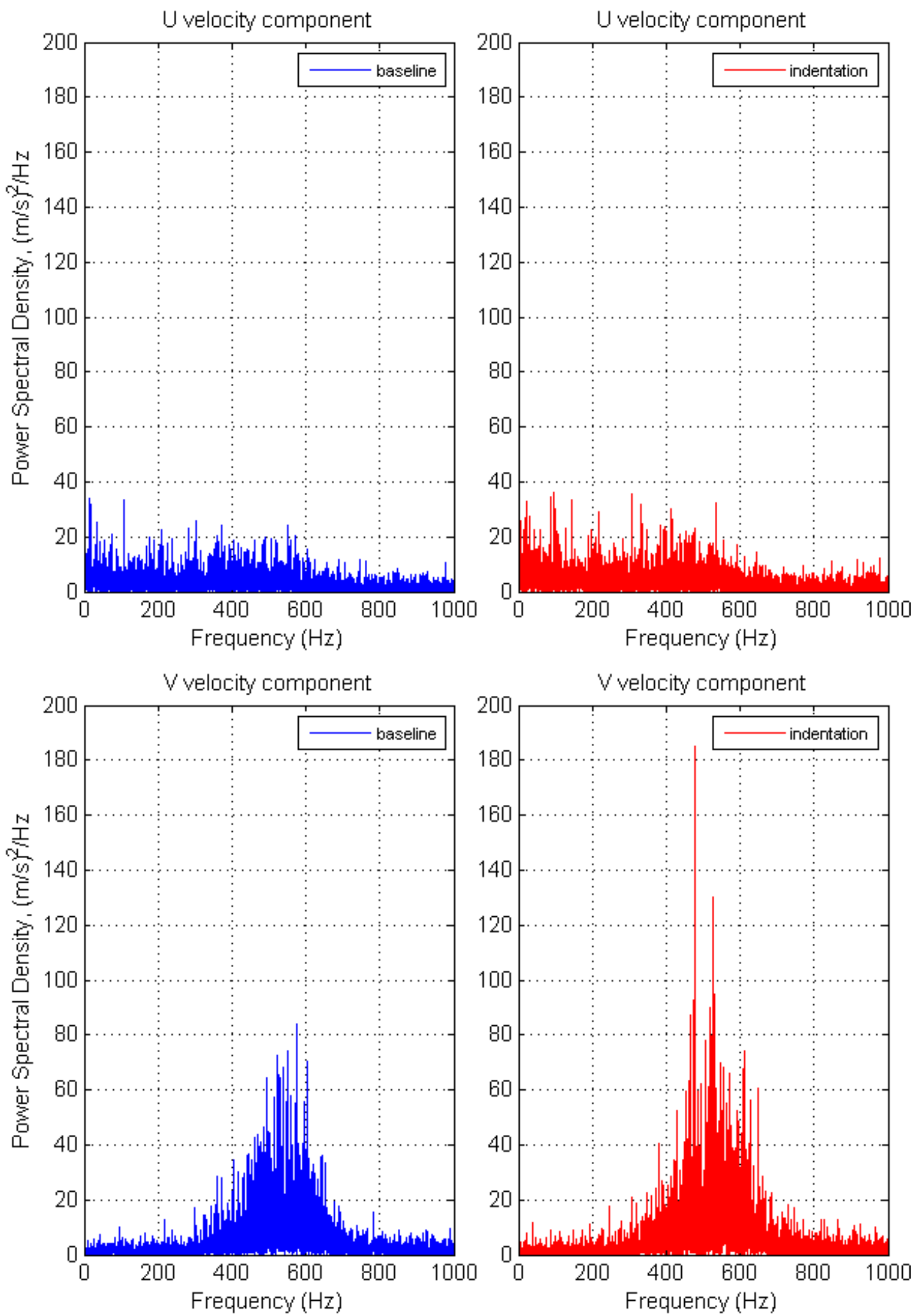

Figure 33. Power spectral analysis at the height of maximum v-component velocity fluctuation at spanwise Station 1 and $\sim 0.250 \mathrm{c}$ downstream of aerofoil trailing edge

American Institute of Aeronautics and Astronautics 

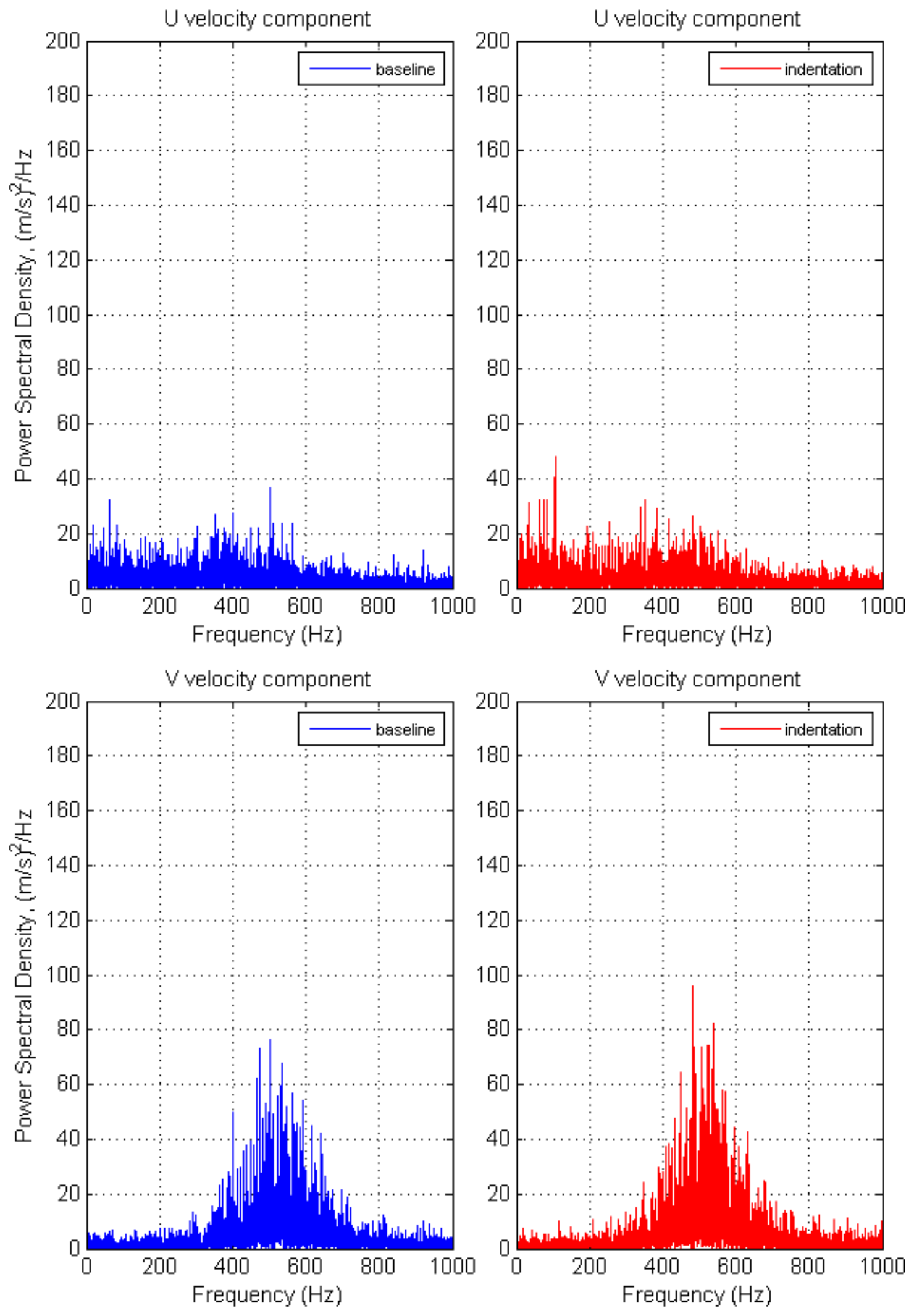

Figure 34. Power spectral analysis at the height of maximum v-component velocity fluctuation at spanwise Station 2 and $\sim 0.250 \mathrm{c}$ downstream of aerofoil trailing edge 

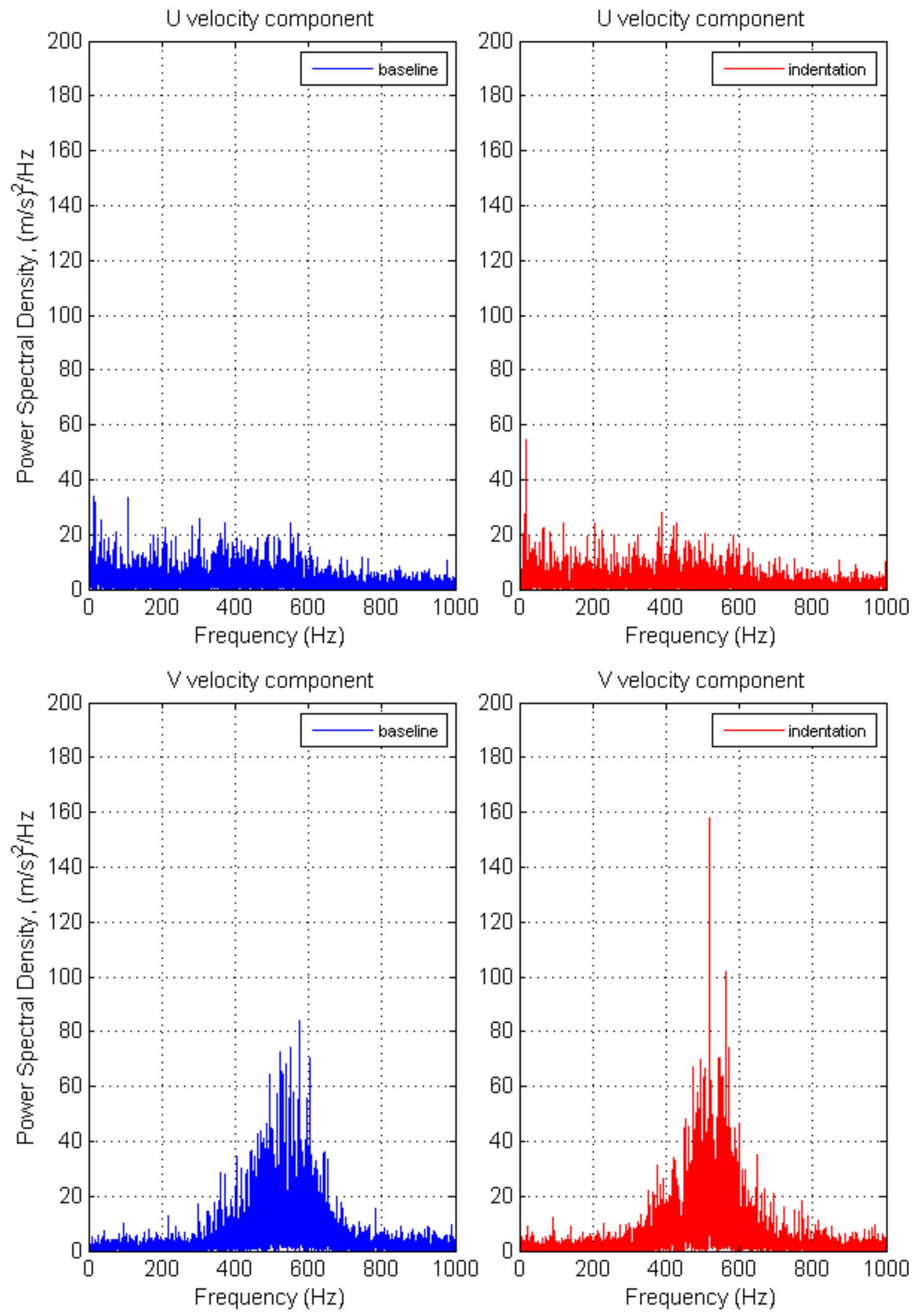

Figure 35. Power spectral analysis at the height of maximum v-component velocity fluctuation at spanwise Station 3 and $\sim 0.250 \mathrm{c}$ downstream of aerofoil trailing edge 


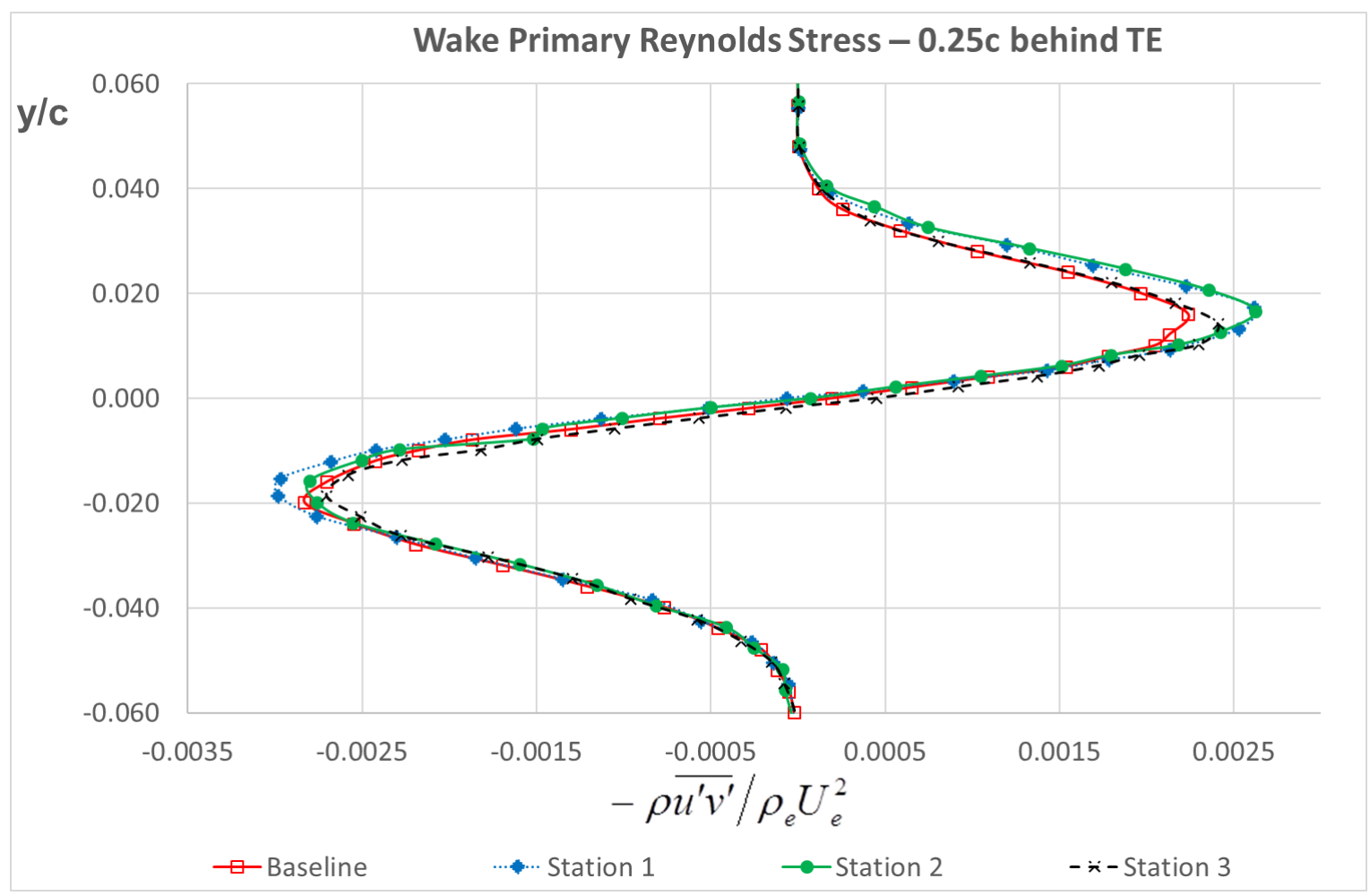

Figure 36. Primary Reynolds-stress profiles at $\sim 0.250 \mathrm{c}$ downstream of aerofoil trailing edge

\section{Conclusions}

1. Although the indentations are located under the long separation bubble at $3^{\circ} \mathrm{AoA}$, they still affect the separated flow considerably. This suggests that the bubble height is less than or comparable to the depth of the indentation. The indentations shorten the bubble by generating up-flow pairs of counter-rotating streamwise vortices.

2. The oil-flow patterns portray that the indentations create a highly $3 \mathrm{D}$ and complex flow-field with discrete surface patterns, such as vortex footprints. The footprints reveal that the vortices converge around Station 1 before persisting along it all the way to the aerofoil's trailing edge. Furthermore, the patterns illustrate that the vortices fragment and shorten the bubble in a uniform pattern across the model span.

3. The indentations reduce the separation bubble by a spanwise-averaged amount of $\sim 0.120 \mathrm{c}(\sim 32.0 \%)$. In addition to shortening the bubble, the indentations also provoke laminar separation, that is, the bubble is shifted upstream by $\sim 0.030 \mathrm{c}$. Thus, the vortices have hastened the flow transition.

4. The altered flow-field and the converging geometry of the indentations modify the pressure distribution at all three spanwise stations in relation to the baseline case. In summary, there are four regions in which the surface pressure has been modified, that is, augmentation of the leading-edge suction due to the shorter and shifted separation bubble, the high-pressure region around and adjacent to the front portion of the indentation followed by a low-pressure region around and adjacent to the indentation apex, and a high-pressure region caused by the earlier turbulent reattachment.

5. Although the indentations shorten the bubble and shift it upstream, the improvement to the pressure distribution at Station 1 (in the form of stronger leading-edge suction) is limited by the high-pressure region within the front area of the indentation (flow blockage), removal of some of the surface curvature by the indentation, and a weaker pressure recovery due to the up-flow region of a pair of vortices at the same station. Therefore, there is no measurable change in $\mathrm{c}_{\mathrm{l}, \mathrm{p}}$ and $\mathrm{c}_{\mathrm{d}, \mathrm{p}}$ at Station 1 . These limiting factors are not present, or at least shorter in streamwise extent and / or less intensive in the case of the high pressure region, in the region adjacent to the indentations, i.e., at Stations 2 and 3. Furthermore, the separation bubble has been shortened by a similar amount across the span. Additionally, at Stations 2 and 3, the bubble has been 
fragmented into two separate shorter bubbles, whose height and adverse effect on the pressure distribution should be less compared to those of the single bubble at Station 1. Thus, the leading-edge suction at Stations 2 and 3 improves by an adequate amount to provide an increase in $\mathrm{c}_{1, \mathrm{p}}$ that is greater than its uncertainty range. The combination of the augmented leading-edge suction with the high-pressure region aft of the point at which the pressure force is directed vertically also leads to a measurable improvement in $\mathrm{c}_{\mathrm{d}, \mathrm{p}}$ at Station 3.

6. The spanwise-averaged $c_{1, p}$ and $c_{d, p}$ of the indented model is better than the corresponding baseline value by at least $\sim 1.3 \%$ and $\sim 0.3 \%$, respectively. Despite this improvement, it is not known if the aerodynamic efficiency of the model has increased at this AoA. This is because the near-wake results show higher turbulence intensity and turbulent mixing (Reynolds stress) due to the streamwise vortices, which implies that viscous drag on the model has increased. Thus, the convergent indentations are effective in reducing the size of the LSB, but the creation of other competing effects prohibits an anticipated reflection in $\mathrm{c}_{1, \mathrm{p}}$ and $\mathrm{c}_{\mathrm{d}, \mathrm{p}}$ at a $\operatorname{Re}_{\text {corr }}$ of $\sim 2.70 \times 10^{5}$.

7. The dimensional turbulent fluctuations and mixing in the near wake are highest at Station 1. This is possibly due to the mutual interference and common up-flow region between the pair of counter-rotating vortices at this station. Furthermore, PSD analysis shows that the v-component fluctuations also have the highest energy content at Station 1. The fluctuations and turbulent mixing decrease towards Station 3, thus showing that the near wake has not fully mixed, that is, it is still 3D.

8. PSD analysis just upstream of the reattachment location shows that the separation frequency is $\sim 16 \mathrm{~Hz}$, that is, the St of the dominant fluctuations in the separation bubble is $\sim 0.25$. Furthermore, PSD analysis also shows that reattachment in the indented case is associated with fluctuations of higher energy content compared to that in the baseline case.

9. At Station 1, reattachment occurs at the indentation apex where the surface pressure has been considerably reduced. Since this is a contrary effect to what normally occurs around the reattachment location, that is, impression of high pressure on the surface, the pressure field around the indentation at this AoA is dominated by its converging geometry rather than the presence of the prevailing bubble over it.

10. Comparison with experiments performed by other researchers at similar test conditions shows that the convergent indentations used in this study perform better than a spanwise groove, but not as well in comparison to protrusions, that is, wishbone VGs.

\section{Future Work}

1. For the current case, the total lift and drag should be measured.

2. A parametric study of the different length scales of the indentations, that is, depth, sweep angle, chordwise location, and spacing, should be performed in an endeavour to identify an optimum geometry for the indentations.

3. The effects of different Re and pressure gradients on the performance of the indentations should also be studied.

\section{References}

\footnotetext{
${ }^{1}$ Thomas, R. H., Choudhari, M. M., and Joslin, R. D., "Flow and Noise Control: Review and Assessment of Future Directions," NASA TM-2002-211631, 2002.

${ }^{2}$ Yao, C.-S., Lin, J. C., and Allan, B. G., "Flow-Field Measurement of Device-Induced Embedded Streamwise Vortex on a Flat Plate," 1st AIAA Flow Control Conference, AIAA Paper 2002-3162, AIAA, Reston, VA, 2002.

${ }^{3}$ Lin, J. C., "Review of Research on Low-Profile Vortex Generators to Control Boundary-Layer Separation," Progress in Aerospace Sciences, Vol. 38, No. 4-5, 2002, pp. 389-420.

${ }^{4}$ Gad-el-Hak, M., Flow Control: Passive, Active, and Reactive Flow Management, Cambridge University Press, Cambridge, 2000.

${ }^{5}$ Robarge, T. W., Stark, A. M., Min, S.-K., Khalatov, A. A., and Byerley, A. R., "Design Considerations for Using Indented Surface Treatments to Control Boundary Layer Separation," 42nd AIAA Aerospace Sciences Meeting and Exhibit, AIAA Paper 2004-425, AIAA, 2004.

${ }^{6}$ Kerho, M., Hutcherson, S., Blackwelder, R. F., and Liebeck, R. H., "Vortex Generators Used to Control Laminar Separation Bubbles," Journal of Aircraft, Vol. 30, 1993, pp. 315-319.

${ }^{7}$ Lissaman, P. B. S., "Low-Reynolds-Number Airfoils," Annual Review in Fluid Mechanics, Vol. 15, 1983, pp. $223-239$.

${ }^{8}$ Massey, B. S., Mechanics of Fluids, $7^{\text {th }}$ ed., Stanley Thornes, Cheltenham, 1998.

${ }^{9}$ Tani, I., "Low-Speed Flows Involving Bubble Separations," Progress in Aeronautical Sciences, Vol. 5, 1964, pp. $70-103$.
} 
${ }^{10}$ Shah, N. K., "Boundary Layer Flow Control Using Surface Indentations," M.Res. Thesis, Department of Aerospace Sciences, Cranfield University, Cranfield, U.K., 2004.

${ }^{11}$ Barlow, J. B., Rae, W. H., and Pope, A., Low Speed Wind Tunnel Testing, $3^{\text {rd }}$ ed., John Wiley and Sons, New York, 1999.

${ }^{12}$ McAlister, K. W. and Takahashi, R. K., "NACA 0015 Wing Pressure and Trailing Vortex Measurements," NASA TP3151, 1991.

${ }^{13}$ Browne, L. W. B., Antonia, R. A., and Chua, L. P., "Calibration of X-probes for Turbulent Flow Measurements," Experiments in Fluids, Vol. 7, 1989, pp. 201-208.

${ }^{14}$ Schlichting, H. and Gersten, K., Boundary-Layer Theory, $8^{\text {th }}$ revised and enlarged ed., Springer, Berlin, 2001.

${ }^{15}$ Bertin, J. J. and Cummings, R. M., Aerodynamics for Engineers, $5^{\text {th }}$ ed., Pearson Educational, Upper Saddle River, N.J., 2009.

${ }^{16}$ Abbott, I. H. and Von Doenhoff, A. E., Theory of Wing Sections: Including a Summary of Airfoil Data, Dover, New York, 1959.

${ }^{17}$ Lin, J. C., Robinson, S. K., McGhee, R. J., and Valarezo, W. O., "Separation Control on High-Lift Airfoils via MicroVortex Generators," Journal of Aircraft, Vol. 31, 1992, pp. 1317-1323.

${ }^{18}$ Wendt, B. J., Greber, I., and Hingst, W. R., "The Structure and Development of Streamwise Vortex Arrays Embedded in a Turbulent Boundary Layer," 30th AIAA Aerospace Sciences Meeting and Exhibit, AIAA Paper 1992-0551, AIAA, Washington, D.C., 1992.

${ }^{19}$ Shan, H., Jiang, L., Liu, C., Love, M., and Maines, B., "Numerical Study of Passive and Active Flow Separation Control over a NACA0012 Airfoil," Computers \& Fluids, Vol. 37, 2008, pp. 975-992.

${ }^{20}$ Weygandt, J. H. and Mehta, R. D., "Three-dimensional Structure of Straight and Curved Plane Wakes," Journal of Fluid Mechanics, Vol. 282, 1995, pp. 279-311.

${ }^{21}$ Amir, M., "Active Flow Control Studies at Subsonic Speeds," Ph.D. Thesis, Department of Mechanical, Aerospace, and Civil Engineering, The University of Manchester, Manchester, U.K., 2007. 